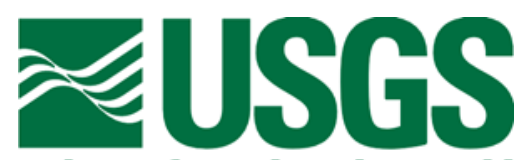

science for a changing world

Prepared in cooperation with the

OKLAHOMA DEPARTMENT OF TRANSPORTATION

\title{
Flood Frequency Estimates and Documented and Potential Extreme Peak Discharges in Oklahoma
}

Water-Resources Investigations Report 01-4152

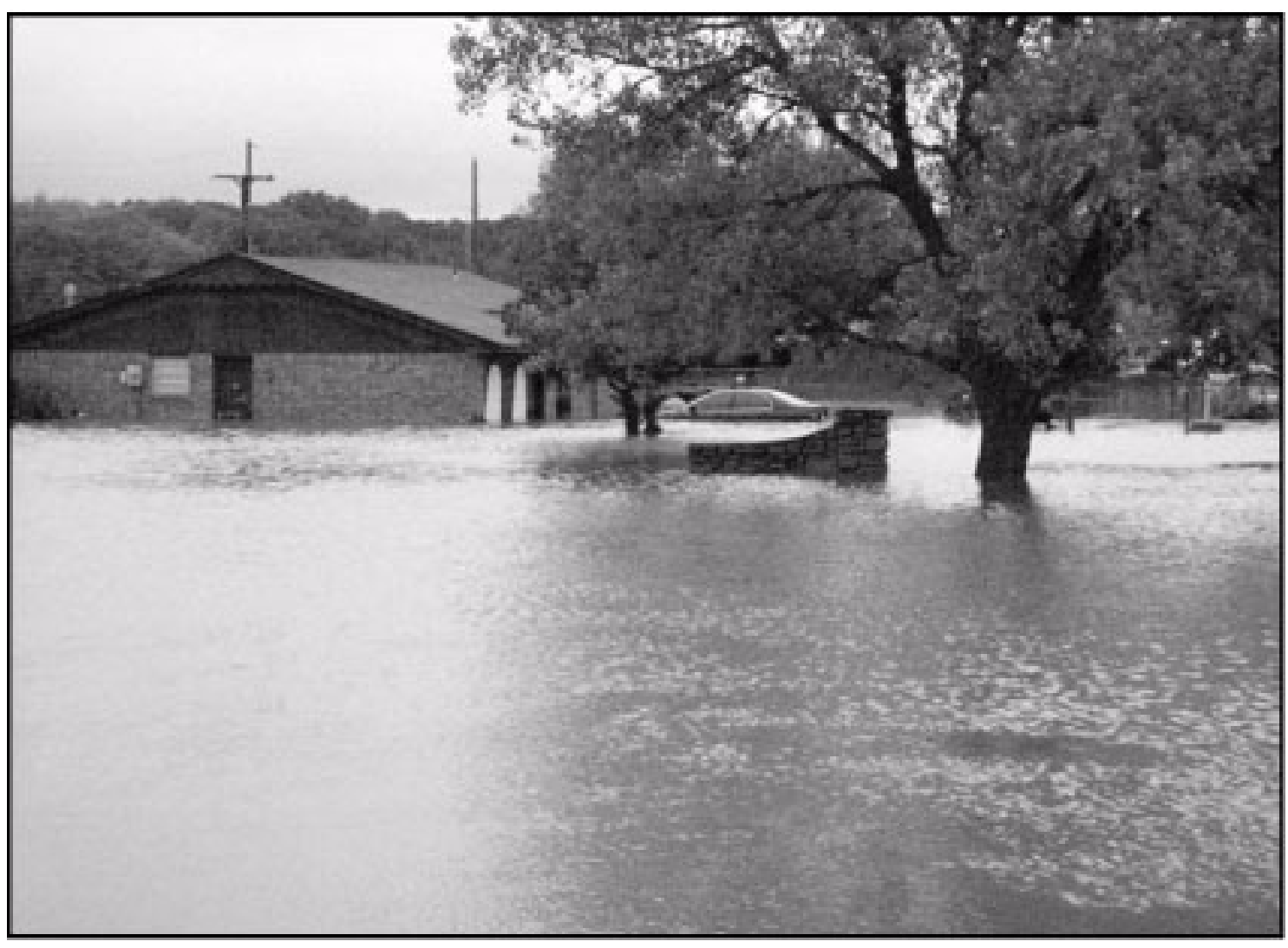

U.S. Department of the Interior

U.S. Geological Survey 
Cover: Photograph was taken October 23, 2000, during the Apache, Oklahoma, flood. Photographer: Stanley Wright, The Apache News. 
Flood Frequency Estimates and Documented and Potential Extreme Peak Discharges in Oklahoma

By Robert L. Tortorelli and Lan P. McCabe

In Cooperation with the Oklahoma Department of Transportation

Water-Resources Investigations Report 01-4152 


\title{
U.S. Department of the Interior Gale A. Norton, Secretary
}

\author{
U.S. Geological Survey \\ Charles G. Groat, Director
}

U.S. Geological Survey, Reston, Virginia: 2001

For sale by U.S. Geological Survey, Information Services

Box 25286, Denver Federal Center

Denver, CO 80225

District Chief

U.S. Geological Survey

202 NW 66 St., Bldg. 7

Oklahoma City, OK 73116

For more information about the USGS and its products:

Telephone: 1-888-ASK-USGS

World Wide Web: http://www.usgs.gov/

Information about water resources in Oklahoma is available on the World Wide Web at

http://ok.water.usgs.gov

Any use of trade, product, or firm names in this publication is for descriptive purposes only and does not imply endorsement by the U.S. Government.

Although this report is in the public domain, it contains copyrighted materials that are noted in the text.

Permission to reproduce those items must be secured from the individual copyright owners. 


\section{Contents}

Abstract. $\ldots \ldots \ldots \ldots \ldots \ldots \ldots \ldots \ldots \ldots \ldots \ldots \ldots \ldots \ldots \ldots \ldots \ldots \ldots \ldots \ldots \ldots \ldots \ldots \ldots \ldots \ldots \ldots \ldots 1$

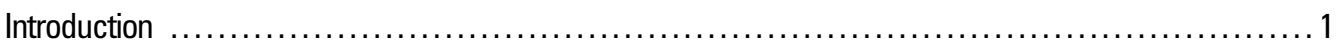

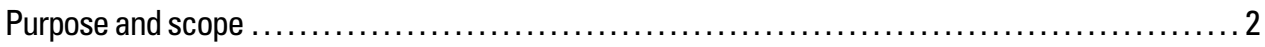

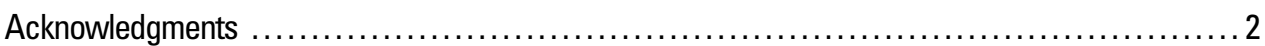

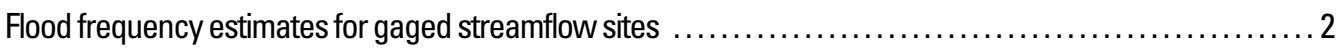

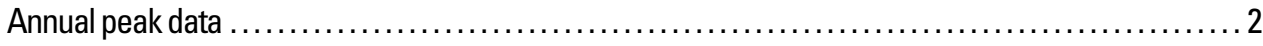

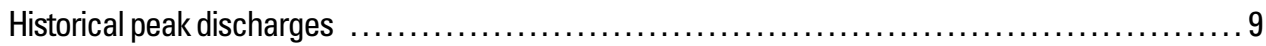

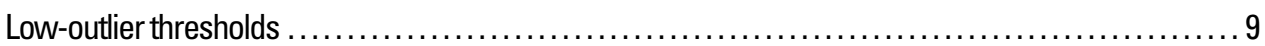

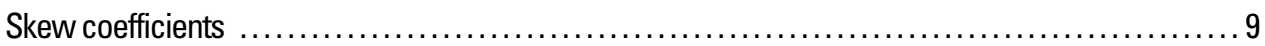

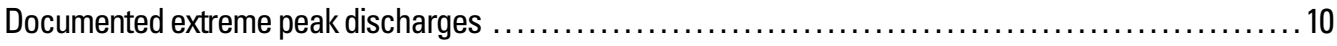

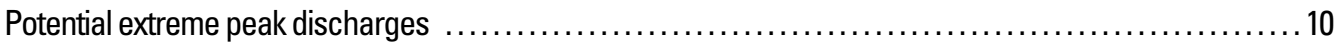

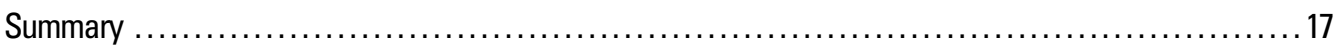

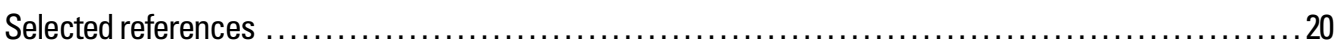

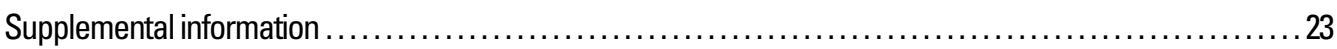

Table 1. Documented and potential extreme peak discharges and flood frequency estimates for selected streamflow-gaging stations with at least 8 years of annual peak-discharge data from unregulated, regulated, and urban basins within and near Oklahoma . . . . . . . . 24

Table 2. Documented and potential extreme peak discharges for selected indirect measurement sites without streamflow-gaging stations and streamflow-gaging stations with short periods in basins within Oklahoma

\section{Figures}

1-2. Maps showing:

1. Location of streamflow-gaging stations with at least 8 years of peak-discharge data used

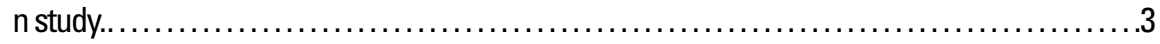

2. Location of miscellaneous indirect measurement sites and streamflow-gaging stations

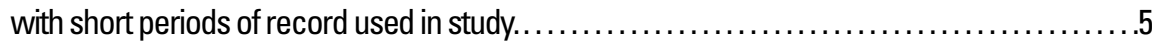

3-4. Graphs showing:

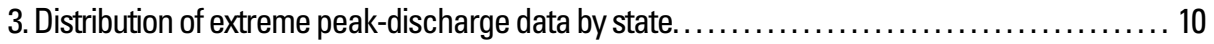

4. Distribution of extreme peak-discharge data by major drainage basins. $\ldots \ldots \ldots \ldots \ldots \ldots \ldots 11$ 5-6. Maps showing:

5. Oklahoma Peak Discharge Envelope Curve based on peak-discharge measurements at streamflow sites east of 98 degrees 15 minutes longitude.

6. Oklahoma Peak Discharge Envelope Curve based on peak-discharge measurements at streamflow sites west of 98 degrees 15 minutes longitude. . . . . . . . . . . . . . . 15

7. Comparison of East and West Oklahoma Peak Discharge Envelope Curves. ................. 18 


\section{Tables}

3. Summary of drainage area and state distribution of extreme peak-discharge measurements ........9

4. Oklahoma Peak Discharge Envelope Curve Data ..................................... 19

\section{Conversion Factors and Datum}

\begin{tabular}{lcl}
\hline Multiply & By & To obtain \\
\hline & Length & \\
\hline $\begin{array}{l}\text { inch (in.) } \\
\text { mile (mi) }\end{array}$ & 25.4 & millimeter $(\mathrm{mm})$ \\
& 1.609 & kilometer $(\mathrm{km})$ \\
\hline & Area & \\
\hline \multirow{3}{*}{ square mile $\left(\mathrm{mi}^{2}\right)$} & 2.590 & square kilometer $\left(\mathrm{km}^{2}\right)$ \\
\hline & Flow rate & \\
\hline & 0.02832 & cubic meter per second $\left(\mathrm{m}^{3} / \mathrm{s}\right)$ \\
\hline
\end{tabular}

Vertical coordinate information is referenced to North American Vertical Datum of 1988 (NAVD 88).

Horizontal coordinate information is referenced to North American Datum of 1983 (NAD 83).

Sea level: In this report "sea level" refers to the National Geodetic Vertical Datum of 1929 (NGVD of 1929) - a geodetic datum derived from a general adjustment of the first-order level nets of both the United States and Canada, formerly called Sea Level Datum of 1929. 


\title{
Flood Frequency Estimates and Documented and Potential Extreme Peak Discharges in Oklahoma
}

\author{
By Robert L. Tortorelli and Lan P. McCabe
}

\section{Abstract}

Knowledge of the magnitude and frequency of floods is required for the safe and economical design of highway bridges, culverts, dams, levees, and other structures on or near streams; and for flood plain management programs. Flood frequency estimates for gaged streamflow sites were updated, documented extreme peak discharges for gaged and miscellaneous measurement sites were tabulated, and potential extreme peak discharges for Oklahoma streamflow sites were estimated. Potential extreme peak discharges, derived from the relation between documented extreme peak discharges and contributing drainage areas, can provide valuable information concerning the maximum peak discharge that could be expected at a stream site. Potential extreme peak discharge is useful in conjunction with flood frequency analysis to give the best evaluation of flood risk at a site.

Peak discharge and flood frequency for selected recurrence intervals from 2 to 500 years were estimated for 352 gaged streamflow sites. Data through 1999 water year were used from streamflow-gaging stations with at least 8 years of record within Oklahoma or about 25 kilometers into the bordering states of Arkansas, Kansas, Missouri, New Mexico, and Texas. These sites were in unregulated basins, and basins affected by regulation, urbanization, and irrigation.

Documented extreme peak discharges and associated data were compiled for 514 sites in and near Oklahoma, 352 with streamflow-gaging stations and 162 at miscellaneous measurements sites or streamflow-gaging stations with short record, with a total of 671 measurements. The sites are fairly well distributed statewide, however many streams, large and small, have never been monitored.

Potential extreme peak-discharge curves were developed for streamflow sites in hydrologic regions of the state based on documented extreme peak discharges and the contributing drainage areas.

Two hydrologic regions, east and west, were defined using 98 degrees 15 minutes longitude as the dividing line.

\section{Introduction}

Knowledge of the magnitude and frequency of floods is required for the safe and economical design of highway bridges, culverts, dams, levees, and other structures on or near streams. Flood plain management programs and flood-insurance rates also are based on flood magnitude and frequency information. A flood is any relatively high streamflow overtopping the natural or artificial banks in any reach of a stream (Leopold and Maddock, 1954, p. 249-251). The magnitude of a flood is referred to as the flood peak, which is the highest value of the discharge or stage attained by a flood; thus, peak discharge or peak stage (Langbein and Isseri, 1960, p.10). Three kinds of flood frequency analyses may be conducted; (1) peak discharge; (2) peak stage; and (3) total volume (Dalrymple, 1960, p. 5). Peak-discharge flood frequency analyses are the most common and are the type of flood frequency analyses that will be presented in this investigation.

Documented historical peak-discharge data are valuable for giving perspective to flood potential for local communities near a streamflow-gaging site. Often very large floods happened so long ago that people have forgotten or are unaware that the floods happened and could happen again. These documented peak discharges may be much larger than large damaging streamflows that have recently occurred.

The potential extreme peak discharge at a site, which is an estimate of the maximum expected peak discharge that could occur at a stream site, is used in conjunction with flood frequency analysis to give the best evaluation of flood risk at a site. Extreme flood potential exceeds the discharge associated with large recurrence-interval flood, such as the 100-year peak discharge (Asquith and Slade, 1995). Potential extreme peak-discharge curves, derived from the relation between documented extreme peak-discharge measurements and contributing drainage areas from a hydrologic region, are not associated with specific probabilities or frequencies, but give evidence as to the magnitude of flow that has occurred and can occur. Given similar basin characteristics, a peak lying close to the envelope curve might occur at other basins in the same region (Crippen, 1982). The U. S. Geological Survey (USGS), in cooperation with the Oklahoma Department of Transportation, conducted an investigation to define the potential extreme peak discharges in Oklahoma. 


\section{Flood Frequency Estimates and Documented and Potential Extreme Peak Discharges in Oklahoma}

\section{Purpose and Scope}

The purpose of this report is to: (1) update flood frequency estimates for gaged streamflow sites with 8 years or more of record for unregulated, regulated, and urban basins in and near Oklahoma, using data through 1999 water year; (2) present documented extreme peak discharges for gaged and miscellaneous measurement sites; (3) present potential extreme peak-discharge curves for unregulated basins for the state; and (4) present potential extreme peak-discharge estimates for all the streamflow measurement sites used in this investigation.

The potential extreme peak-discharge curves were developed based on documented extreme peak-discharge measurements from 352 streamflow-gaging stations in Oklahoma and within about 25 kilometers of Oklahoma in the bordering states of Arkansas, Kansas, Missouri, New Mexico, and Texas (fig. 1; table 1, back of report); and 162 sites in Oklahoma at miscellaneous measurement sites without streamflow-gaging stations, or streamflow-gaging stations with short record (fig. 2; table 2, back of report). The peak-discharge measurements presented are from unregulated basins, and basins affected by regulation, urbanization, and irrigation. An unregulated basin is defined as a drainage basin for which the peak discharges are not affected by regulation, reservoirs, diversions, urbanization, or other human-related activities. Significant regulation by dams or other manmade modification of streamflow is defined as 20 percent or more of the contributing drainage basin being affected (Heimann and Tortorelli, 1988).

This report updates the flood frequencies presented in Heimann and Tortorelli (1988). This update can be used to estimate flood discharges for Oklahoma streamflow-gaging sites with a drainage area greater than 2,510 square miles, because it includes 15 years of additional annual peak data and records from many additional gaging stations, including major peak discharges recorded during 1987, 1990, 1993, and 1995 water years. This report also includes and updates the flood frequencies in Tortorelli (1997), which estimated flood discharges for Oklahoma streamflow-gaging sites with drainage areas less than or equal to 2,510 square miles.

This report also updates the potential extreme peak-discharge analysis by Crippen and Bue (1977) for Oklahoma.

\section{Acknowledgments}

The following U.S. Geological Survey personnel provided assistance with this report: Darrell Walters and Tony Coffey provided accurate and valuable information about historic streamflow-gaging data; William Asquith provided guidance about the investigation methodology; and Michael Stallings and Jason Masoner produced the streamflow-gaging station site maps.

\section{Flood Frequency Estimates for Gaged Streamflows}

The curvilinear relation between flood peak magnitude and annual exceedance probability or recurrence interval is referred to as a flood frequency curve. Annual exceedance probability is the probability of a given flood magnitude being equaled or exceeded in any one year. Recurrence interval is the reciprocal of the annual exceedance probability, and represents the average number of years between peak flow exceedances of that magnitude. For instance, a flood having an annual exceedance probability of 0.01 has a recurrence interval of 100 years. This does not imply that a 100-year flood peak will be equaled or exceeded each 100 years, but that it will be equaled or exceeded on the average of once every 100 years (Thomas and Corley, 1977). That peak might be exceeded in successive years, or more than once in the same year. The probability of that peak happening is called risk. Procedures for making flood risk estimates are given by the Interagency Advisory Committee on Water Data (IACWD) (1982).

The IACWD (1982) provides a standard procedure for flood frequency estimation using the log-Pearson Type III (LPIII) distribution. The procedure uses systematically collected and historical peak-discharge values to define frequency distribution. The shape of the distribution is defined by a skew coefficient used in the estimation procedure.

The LPIII distribution does not always define a suitable distribution of peak-discharge values because of variation in the climatic and physiographic characteristics in the basin. The data distribution is defined by Weibull plotting positions (Chow and others, 1988). An inappropriate fit of the LPIII distribution to the distribution of peak-discharge data can produce erroneous values for flood frequency. Therefore, for the estimation of flood frequency in this investigation, available historical flood information, low-outlier thresholds, and skew coefficients were all considered, following the IACWD guidelines. LPIII flood frequency estimates of the 2-, 5-, 10-, 25-, 50-, 100-, and 500-year floods are given for each gaged station used in this investigation in table 1 (back of report).

\section{Annual Peak Data}

All pertinent annual peak-discharge data were collated and reviewed to begin the flood frequency analysis. This review of data eliminated discrepancies across state lines and accounted for data in the immediate bordering areas of a state with similar hydrology.

The station flood frequency analysis presented is based on annual peak-discharge data systematically collected at 352 gag- 


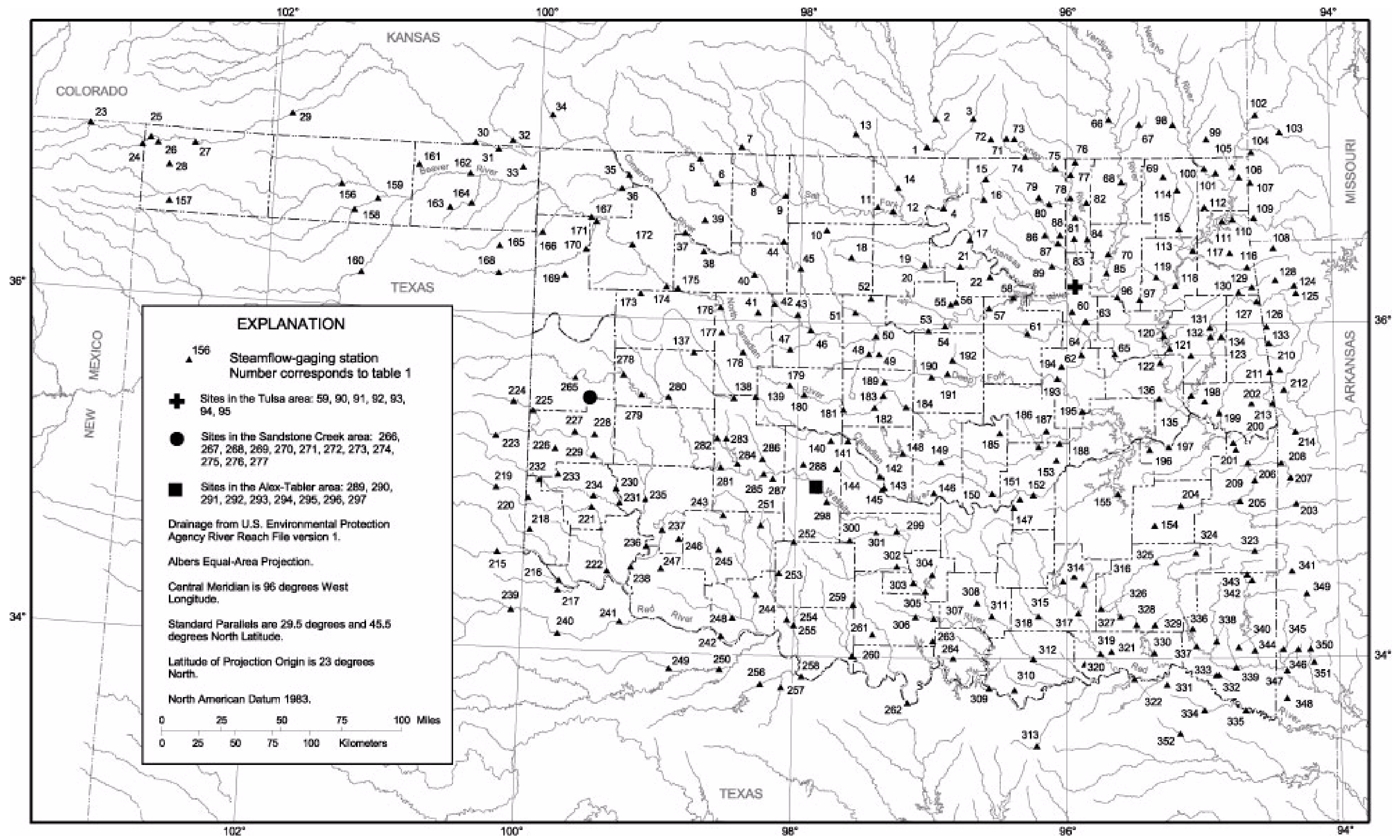

Figure 1. Location of streamflow-gaging stations with at least 8 years of peak-discharge data used in study. 


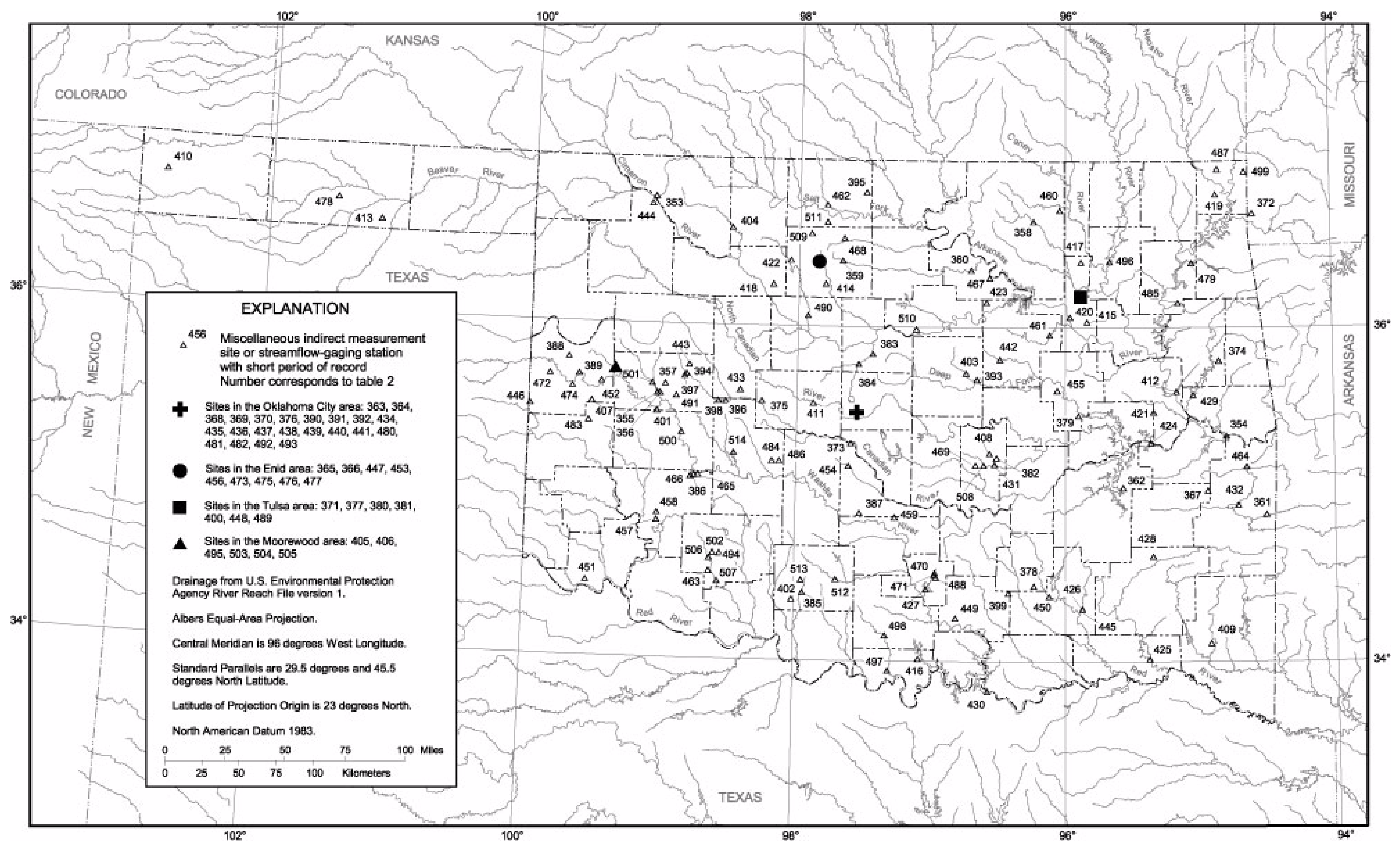

Figure 2. Location of miscellaneous indirect measurement sites and streamflow-gaging stations with short periods of record used in study. 
ing stations (fig. 1; table 1, back of report). Those data were based on a water year, October 1 through September 30. Those data were collected through September 30, 1999, for all stations used in this investigation. Only those stations with at least 8 years of flood peak data were used in the analysis. The IACWD (1982) recommends using at least 10 years of data to make these calculations. The only time stations with less than 10 years of data were used was to fill regional gaps; twelve crest-stage partial record sites (sites 16, 40, 79, 110, 117, 144, 198, 200, 218, $247,314,324$ ) and eight continuous record sites (sites 61, 136, 165, 191, 283, 285, 291, 342) (fig.1: table 1, back of report).

All station data were divided into appropriate periods of record, those periods in which the basins were unregulated, and those periods in which there were substantial effects from regulation by major dams or floodwater retarding structures and other manmade modifications. Therefore, each basin condition was analyzed separately if 8 or more years of record were available.

\section{Historical Peak Discharges}

In addition to the systematically collected peak-discharge data from gaging stations, the USGS routinely compiles, through newspaper accounts and interviews with local residents, information about historical peak discharges and historical peak stages, so that historical peak elevations can be determined for sites or times without measured data. A historical peak discharge is the highest peak discharge since a known date and may precede the installation of the station; a historical peak discharge can occur either before or after installation of a station. Historical information is critical for evaluating flood frequency estimates for the larger recurrence intervals. Many historical peak discharges are associated with catastrophic storms. Large storms can cause flood peaks exceeding those that can be estimated accurately by analyses of available precipitation or annual peak-discharge data.

Historical peak-discharge data also are valuable for giving perspective to flood potential for local communities near a streamflow-gaging site without the need to attach a statistical meaning to the flood. Often very large peak discharges, both historical peak discharges and systematically collected peak discharges, have occurred so long in the past that people have forgotten or are unaware that the floods have occurred. These peak discharges may be much larger than recent large notable floods. For example, the residents of Blackwell, Oklahoma, experienced a large flood on the Chikaskia River (site 14, fig.1; table 1 , back of report) with a peak discharge of 60,700 cubic feet per second on November 1, 1998, when the river rose about 31 feet in less than two days. However, historic records show that there have been larger peak discharges. The largest is a historical peak discharge of 100,000 cubic feet per second on June 10,1923 , before the streamflow gage was installed. The second largest flood was on June 22, 1942, after the gage was installed, when the peak discharge was 85,000 cubic feet per second, almost 50 percent more flow than the 1998 flood; three other peak discharges exceeded the 1998 peak discharge.

Historical peak-discharge data are available for over 20 percent of the 352 Oklahoma and border-state stations. These peaks are designated with an " $\mathrm{H}$ " in table 1 (back of report). Historical peak discharge is included in frequency estimates by the specifying of a high-outlier threshold and historical record length according to guidelines in the IACWD (1982).

Historical information from nearby streamflow gages was used for a small number of stations, including time of large peaks and period of record. These stations are indicated by the footnotes in table 1 (back of report). For many of these stations, usually those with short periods of record, one gage-recorded peak discharge is historically important because it is considerably greater than the other peak discharges. Although no official documentation of the historical importance of that peak discharge is available, a historical perspective was developed through consideration of a longer period of record from relevant nearby stations. Such consideration was necessary to produce more realistic flood frequency analyses for these stations.

\section{Low-Outlier Thresholds}

The climatic and physiographic characteristics of some streams in Oklahoma result in extremely small annual peak-discharge values, referred to as low outliers. Typically, low outliers are identified by visually fitting the data to the LPIII distribution curve. The presence of low outliers can substantially affect the distribution curve; therefore, the fit of the LPIII distribution to the data should be adjusted to account for the presence of low outliers. All peak-discharge values below the lowoutlier threshold, including zero, are excluded from the fitting of the LPIII distribution.

The IACWD (1982) guidelines provide a computational procedure for low-outlier threshold selection; however, the IACWD procedure may not produce accurate low-outlier thresholds for some stations. Therefore, the fit of the preliminary LPIII distribution to the distribution of the peak-discharge data for each station was visually inspected and some stations were assigned a revised low-outlier threshold based on that inspection.

\section{Skew Coefficients}

The IACWD (1982) guidelines recognize three types of skew coefficients: (1) the station skew coefficient calculated from only the systematic record with appropriate adjustments for high and low outliers, if applicable; (2) the generalized skew coefficient from a locally developed generalized skew map or the IACWD (1982) generalized skew map; and (3) the weighted skew coefficient, calculated by combining the locally developed generalized skew or the IACWD (1982) generalized skew with station skew coefficients.

The station skew coefficient is difficult to estimate reliably for stations with short periods of record. The IACWD (1982) 


\section{Flood Frequency Estimated and Documented and Potential Extreme Peak Discharges in Oklahoma}

recommends applying a weighted skew coefficient to the LPIII distribution. The weighted skew coefficient estimate is calculated by weighting the skew coefficient computed from the peak-discharge data at the station (station skew) and the generalized skew coefficient representative of the surrounding area. A weighted skew coefficient is based on the inverse of the respective mean square errors for each of the station and generalized skew coefficients.

Generalized skew coefficients were determined for Oklahoma (Tortorelli and Bergman, 1985) using adjusted station skew coefficients from stations with at least 20 years of peakdischarge data, streamflow data through 1980, and drainage basin areas greater than 10 square miles and less than or equal to 2,510 square miles. Tortorelli and Bergman (1985) updated the generalized skew coefficients recommended by the IACWD (1982), based on data through 1973. Updating the 1985 Oklahoma generalized skew map was not part of this project. However, a check of the standard error of the generalized skew, using the stations used to develop the generalized skew map and updated streamflow records through 1995, indicated that the standard error value of 0.33 was still valid (Tortorelli, 1997). That standard error value was used to compute weighted skew coefficients using the station and Oklahoma generalized skews for all unregulated basins (designated with a "N" in table 1, back of report) with contributing drainage areas less than or equal to 2,510 square miles.

The IACWD (1982) weighted skew coefficients were used for all unregulated basins (designated with a " $\mathrm{N}$ " in table 1, back of report) with contributing drainage areas greater than 2,510 square miles.

Weighted skew coefficients are not appropriate for stations for which there has been significant effects from regulation by major dams or floodwater retarding structures and other manmade modifications. The station skew coefficient was calculated from only the systematic record with appropriate adjustments for high and low outliers, if applicable, for these types of basins (designated with an "R, $\mathrm{U}$, or I" in table 1, back of report).

\section{Documented Extreme Peak Discharges}

The USGS has monitored and published streamflow data for almost 100 years at streamflow-gaging stations throughout Oklahoma, including compilation of annual peak discharges. The USGS also determines peak discharges for large floods at sites without streamflow-gaging stations, through indirect measurements at miscellaneous streamflow measurement sites. Qualifications are assigned to the peak discharges that document the nature of each peak discharge and provide information regarding regulation, reservoirs, land use, and other characteristics affecting the discharge values.

The documented extreme peak discharge was tabulated for each of 352 sites with streamflow-gaging stations (table 1, back of report). The site number, USGS station number, USGS sta- tion name, type of station, type of record, date and magnitude of the documented extreme peak discharge, magnitude of potential extreme peak discharge (described in next section), contributing drainage area, latitude and longitude of station, hydrologic region, type of basin, and LPIII flood frequency estimates (described in previous section) are presented in table 1. If the documented extreme peak discharge was described in a flood report, that report is noted by a footnote. If a station had more than one type of record, all are presented.

The documented extreme peak discharge also was tabulated at each of 162 selected sites in Oklahoma at miscellaneous measurement sites without streamflow-gaging stations or with streamflow-gaging stations with short periods of record (table 2 , back of report). These data were tabulated by visually inspecting the indirect streamflow measurement files at District office. Some have been reported as a historical peak in table 1 and were not repeated in table 2. Many of these peak discharges are associated with catastrophic storms and represent some of the largest peak discharges for the corresponding contributing drainage areas in the state. The descriptive information listed in table 2 is the same as in table 1, except that table 2 lists stream name or indirect measurement site name in place of USGS station name. A USGS station number was noted only on those sites that had a streamflow-gaging station. No LPIII flood frequency estimates were computed. If the documented extreme peak discharge was reported in a flood report, that report is noted by a footnote. If a station had more than one type of record, all are presented.

The sites are fairly well distributed statewide, however many streams, large and small, have never been monitored. The location of each site with streamflow-gaging stations is shown on figure 1 . The site numbers on the figure refer to those in table 1 , back of report, for sites 1-352. The location of each site without streamflow-gaging stations or streamflow-gaging stations with short periods of record is shown on figure 2 . The site numbers on the figure refer to those in table 2, back of report, for sites 353-514. The distribution of the documented peak-discharge measurements from these sites is listed in table 3. A total of 671 streamflow measurements were used from the 514 sites.

\section{Potential Extreme Peak Discharges}

The documented extreme peak discharges were analyzed to estimate the potential extreme peak discharges for Oklahoma. Curves enveloping the documented extreme peak discharges for different regions of the state were developed as a function of the corresponding contributing drainage areas of the streamflow measurement sites. The relation between documented extreme peak discharge and other basin characteristics, such as channel length and channel slope, were evaluated by Asquith and Slade (1995). They reported that the potential extreme peak discharge correlates better with contributing drainage areas than with other characteristics. Crippen and Bue (1977) and Paul Jordan (USGS, written commun., 2000) also 
Table 3. Summary of drainage area and state distribution of extreme peak discharge measurements

\begin{tabular}{|c|c|c|c|c|c|c|c|}
\hline \multirow{3}{*}{$\begin{array}{l}\text { Contributing drainage } \\
\text { area (square miles) }\end{array}$} & \multicolumn{7}{|c|}{ Number of extreme peak discharge measurements } \\
\hline & \multirow[b]{2}{*}{ Oklahoma } & \multicolumn{5}{|c|}{ Border states } & \multirow[b]{2}{*}{ Total } \\
\hline & & Arkansas & Kansas & Missouri & $\begin{array}{l}\text { New } \\
\text { Mexico }\end{array}$ & Texas & \\
\hline 0.1 to less than 1 & 22 & 4 & 1 & & & & 27 \\
\hline 1 to less than 10 & 115 & 2 & 2 & & & 1 & 120 \\
\hline 10 to less than 100 & 120 & 9 & 4 & 2 & & 2 & 137 \\
\hline 100 to less than 1,000 & 154 & 9 & 9 & 3 & 1 & 13 & 189 \\
\hline 1,000 to less than 10,000 & 119 & 3 & 5 & 1 & & 11 & 139 \\
\hline 10,000 to less than 50,000 & 33 & & 2 & & & 11 & 46 \\
\hline 50,000 or more & 10 & 3 & & & & & 13 \\
\hline Total & 573 & 30 & 23 & 6 & 1 & 38 & 671 \\
\hline
\end{tabular}

report that contributing drainage area is the single most influential basin characteristic to use for determination of potential extreme peak-discharge curves. Therefore, other characteristics were not used in the development of the potential extreme peakdischarge curves for Oklahoma. The envelope curve of discharge data is referred to as potential extreme peak-discharge curve (Asquith and Slade, 1995).

Documented extreme peak discharges 25 kilometers into the bordering states were used to expand the data base of streamflow measurements and to account for data in the immediate bordering areas of a state with similar hydrology. The documented extreme peak discharges were plotted by state to check if the potential extreme peak-discharge curve analysis may be unduly influenced by bordering state data (fig. 3). Only one bordering state data point influenced the analysis, the largest documented extreme peak discharge near Van Buren, Arkansas, (site 214, table 1, back of report), the point at which the Arkansas River flows out of Oklahoma. This point is the upper limit in the east hydrologic region described in succeeding sections.

One possible discriminator for potential extreme peak-discharge curves for the state tested and rejected was dividing the data into the two major drainage basins, the Arkansas River basin and the Red River basin. The documented extreme peak discharges were plotted by major drainage basins (fig. 4) and it was decided by visual inspection that there was not enough difference of discharges between basins to warrant using this criterion. There does not appear to be a meaningful role for statistical testing of documented extreme peak discharges between envelope-curve hydrologic regions (W.F. Kirby, USGS, written commun., 2001); therefore, no statistical test was performed to verify this conclusion.

Another possible discriminator tested and accepted was dividing the data into two sets, east and west of a line roughly corresponding to the 28-inch mean annual precipitation line (Tortorelli, 1997), which divides the state into an east and west region. The documented extreme peak discharges were plotted by dividing the data into two hydrologic regions, east and west, separated by a longitude line, 98 degrees 15 minutes. It was decided by visual inspection that there was a significant difference of discharges between regions, and again no statistical test was performed to verify this conclusion. This was the criterion that was adopted to define two hydrologic regions. The resulting potential extreme peak-discharge curves are shown in figure 5 for the east region and figure 6 for the west region.

Peak-discharge data from all types of basins are presented in the graphs to see what type of peakdischarge measurement records define the potential extreme peak-discharge curves (figs. 5 and 6). The peakdischarge measurements presented are from unregulated basins and basins affected by regulation, urbanization, and irrigation. All extreme peak-discharge measurements, regardless of basin type, are documented in this publication to see if extreme peak-discharge measurements from other than unregulated basins would control, or define the potential extreme peak-discharge curves.

The relation between the estimated 100-year flood frequency discharge and the contributing drainage area for each of the streamflow-gaging stations was plotted 


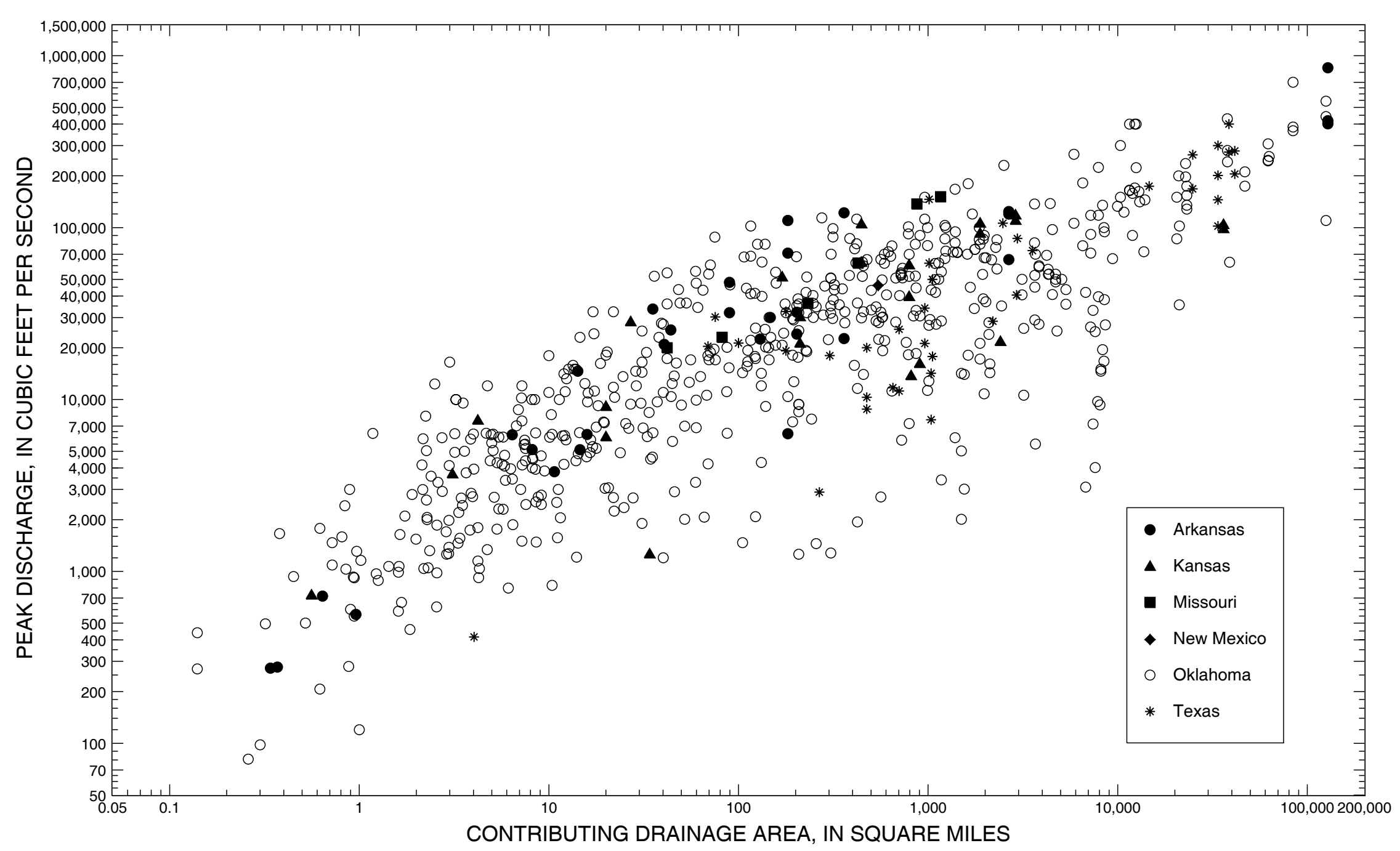

Figure 3. Distribution of extreme peak-discharge data by state. 


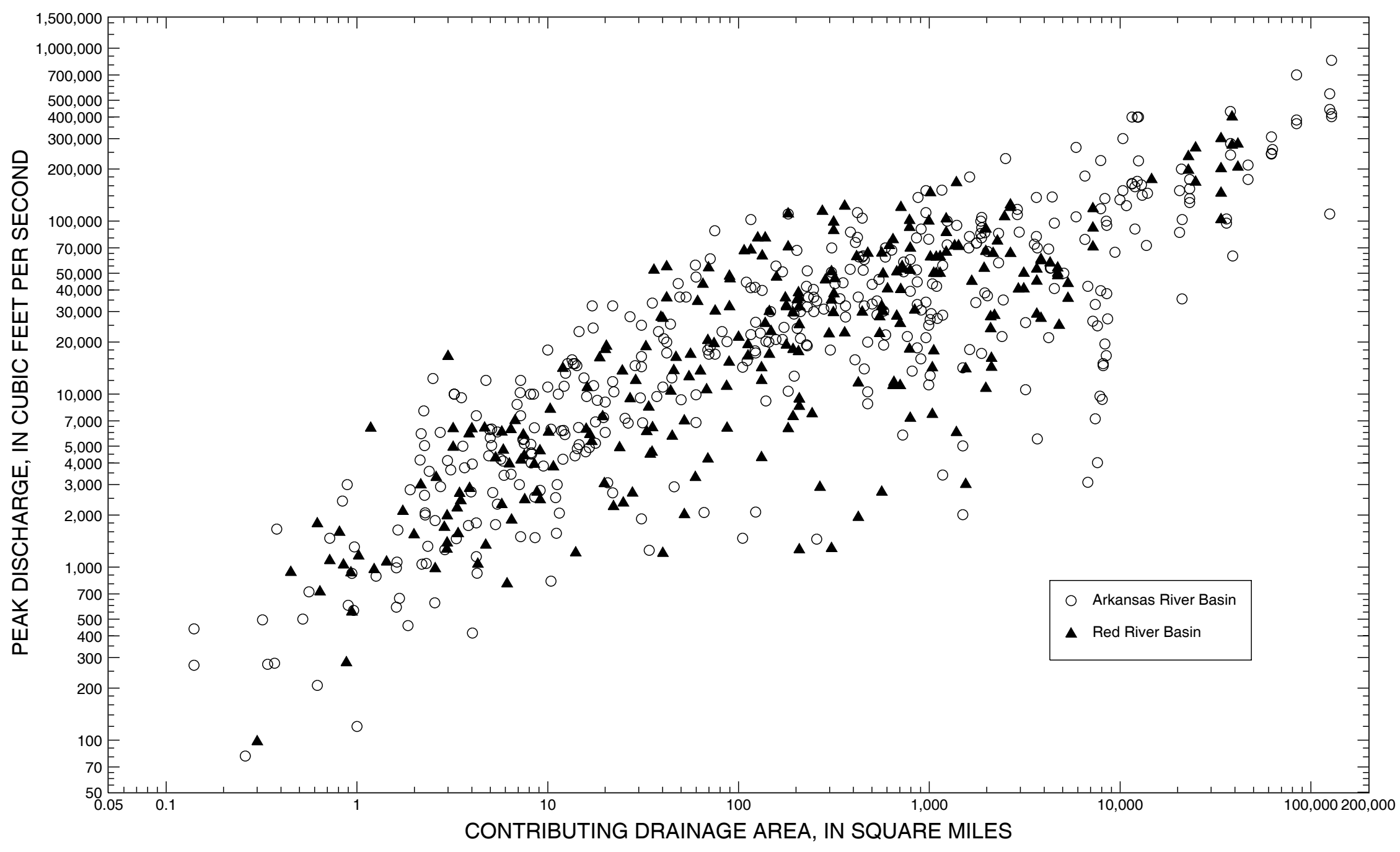

Figure 4. Distribution of extreme peak-discharge data by major drainage basins. 


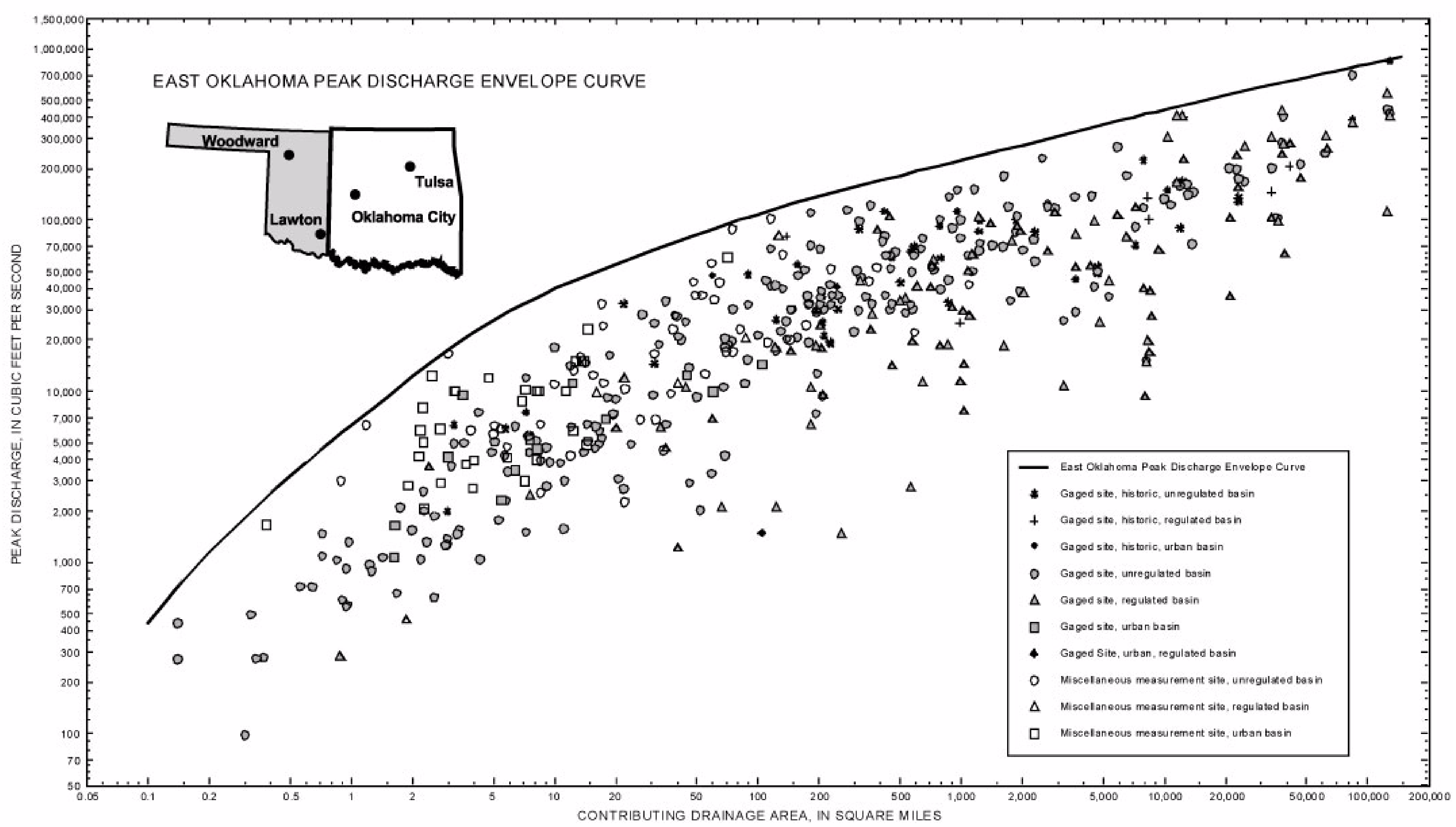

Figure 5. Oklahoma Peak Discharge Envelope Curve based on peak-discharge measurements at streamflow sites east of 98 degrees 15 minutes longitude. 


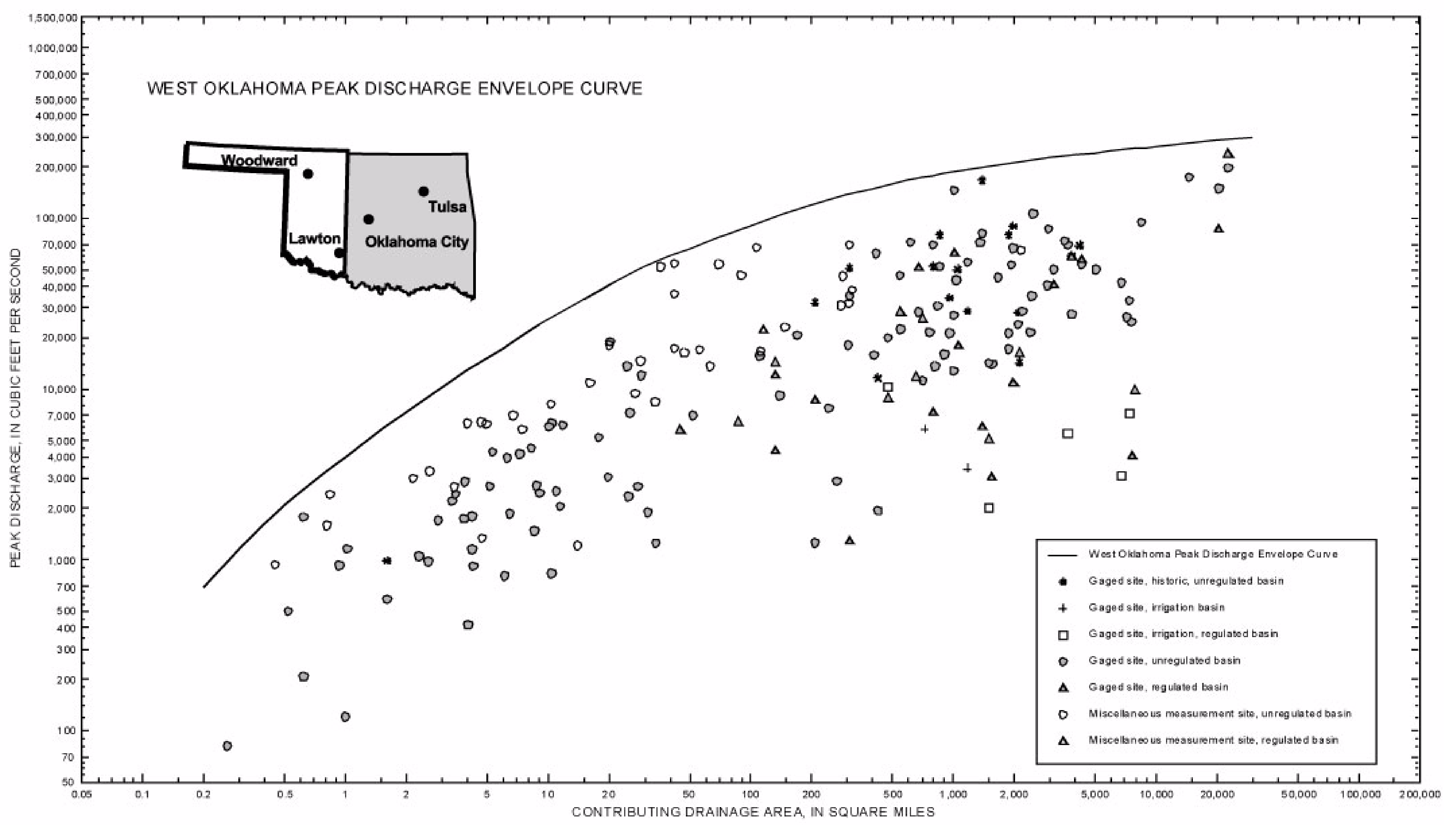

Figure 6. Oklahoma Peak Discharge Envelope Curve based on peak-discharge measurements at streamflow sites west of 98 degrees 15 minutes longitude. 
and used to visually check each of the regional potential extreme peak-discharge curves as suggested by Asquith and Slade (1995). The 100-year peak discharges are listed in table 1 (back of report). These data resulted in the slight upward adjustment of both regional curves in the area below 1.0 square mile and above 1,000 square miles.

The potential extreme peak-discharge curves developed used all peak data as of 1999 water year and will be subject to change as greater peak discharges are subsequently documented. The upward trend of the curves through time is probably due to an increased number of streamflow-gaging stations and an increased period of record (Creager, 1939). However, the rate of increase in peak discharges experienced in the United States has been slowing due to a longer period of recorded data and, perhaps, to approaching geophysical limits (Wolman and Costa, 1984; Matthai, 1969). Longer periods of record also would tend to minimize the effect of weather fluctuations.

Generally, the extreme peak-discharge measurements did define the potential extreme peak-discharge curves in figures 5 and 6 . Miscellaneous measurements of peak discharge in unregulated basins control the curve for drainage basin areas of about 200 square miles and less for the east region; a few miscellaneous measurements of peak discharge in urban basins control the curve for about 5 square miles and less. Miscellaneous measurements of peak discharge in unregulated basins control the curve for drainage basin areas of about 1,000 square miles and less for the west region. The potential extreme peak-discharge curve is defined mostly by measurements of peak discharge in unregulated basins at streamflow-gaging stations in the east region and a few measurements of peak discharge in regulated basins at streamflow-gaging stations and historical peaks, for drainage areas greater than 200 square miles (fig. 5). The potential extreme peak-discharge curve is defined by measurements of peak discharge in unregulated basins at streamflow-gaging stations and historical peaks in the west region for drainage areas greater than 1,000 square miles (fig. 6). One measurement from a regulated basin in the east region was used, Red River near Terral, Okla. (site 258, fig.1; table 1, back of report), in the west region curve. That measurement was used to provide a reasonable upper limit for the curve since most of the drainage area for the site is in the west region. A comparison of the potential extreme peak-discharge curves for two hydrologic regions (figs. 5 and 6) is shown in figure 7.

A potential extreme peak-discharge estimate for any site in a unregulated basin can be obtained from the potential extreme peak-discharge curve for the hydrologic region containing the site, if the contributing drainage area is known. Since all types of drainage basins were used to develop the curves, extreme peak-discharge estimates for sites in which there have been significant effects from manmade modification of streamflow may be obtained if caution is exercised to recognize the limitations of such estimates. For example, streams regulated by major dams are subject to reservoir operations. Urban basins with a high percentage of impervious land cover such as concrete, asphalt and buildings, when coupled with a highly localized storm, could conceivably have higher peak flow. Potential extreme peak-discharge estimates of all 514 sites are listed in tables 1 and 2 (back of report). The curves are presented in tabular form for convenience (table 4). Recurrence intervals cannot be associated with potential extreme peak-discharge estimates because the discharge data do not meet the criteria for statistical analysis (P.R. Jordan, USGS, written commun., 2001).

\section{Summary}

Knowledge of the magnitude and frequency of floods is required for the safe and economical design of highway bridges, culverts, dams, levees, and other structures on or near streams; and for flood plain management programs. The potential extreme peak discharge at a site, which is an estimate of the maximum expected peak discharge that could occur at a stream site, often is used in conjunction with flood frequency analysis to give the best evaluation of flood risk at a site. Potential extreme peak-discharge curves, derived from the relation between documented extreme peak-discharge measurements and the contributing drainage areas from a hydrologic region, are not associated with specific probabilities or frequencies, but give evidence as to the magnitude of flow that has occurred.

This report: (1) updates flood frequency estimates for gaged streamflow sites with 8 years or more of record for unregulated, regulated, and urban basins in and near Oklahoma, using data through 1999 water year; (2) presents documented extreme peak discharges for gaged and miscellaneous measurement sites; (3) presents potential extreme peak-discharge curves for unregulated basins for the State; and (4) presents potential extreme peak-discharge estimates for all the streamflow measurement sites used in this investigation.

Peak discharge and flood frequency for selected recurrence intervals from 2 to 500 years were determined for 352 gaged streamflow sites. Data through 1999 water year were used from streamflow-gaging stations with at least 8 years 


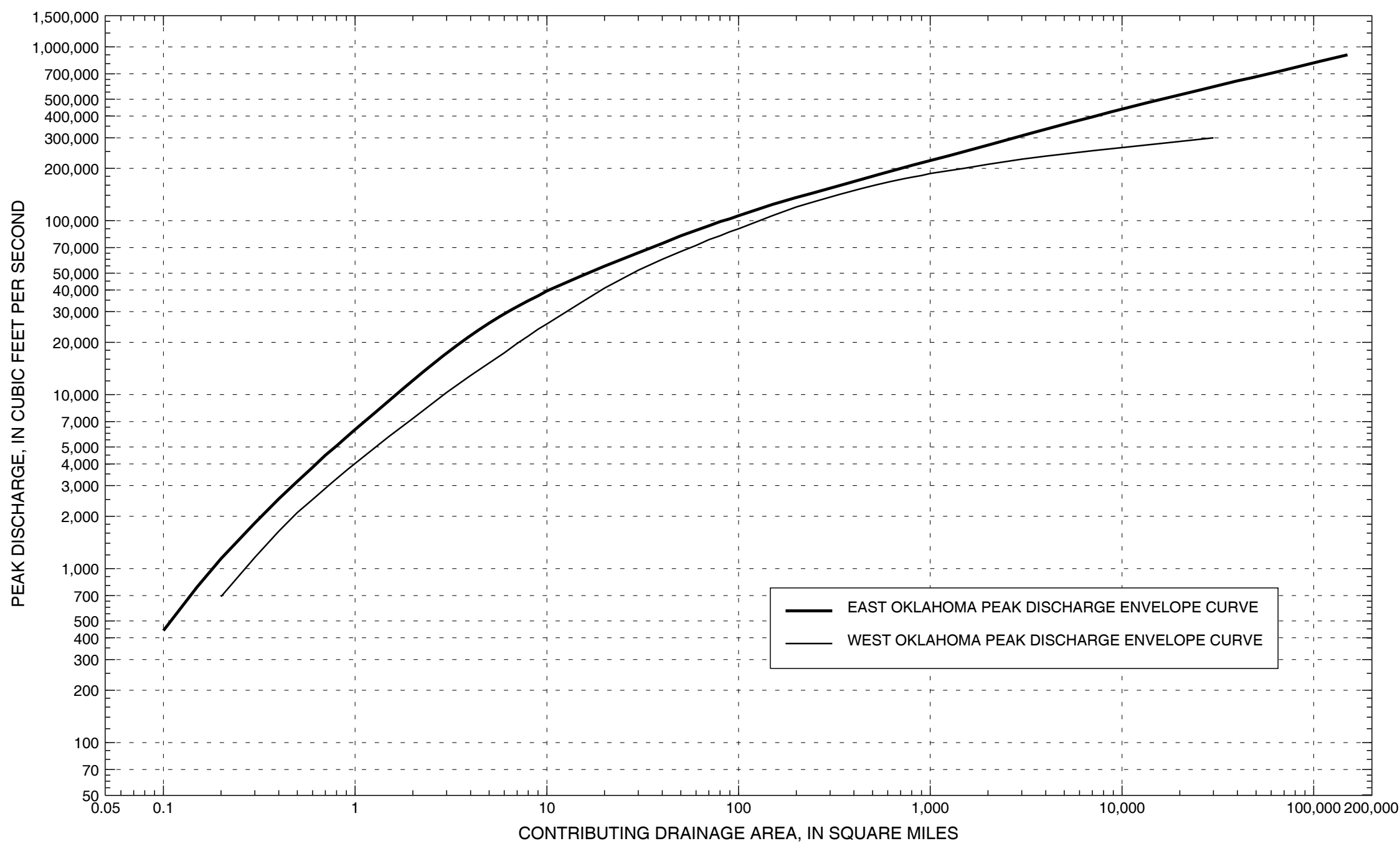

Figure 7. Comparison of East and West Oklahoma Peak Discharge Envelope Curves. 
Table 4. Oklahoma Peak Discharge Envelope Curve Data

[mi ${ }^{2}$, square miles; East, sites east of 98 degrees 15 minutes longitude; West, sites west of 98 degrees 15 minutes longitude]

\begin{tabular}{|c|c|c|c|c|c|}
\hline \multirow{2}{*}{$\begin{array}{l}\text { Contributing } \\
\text { drainage area } \\
\left(\mathrm{mi}^{2}\right)\end{array}$} & \multicolumn{2}{|c|}{ Peak discharge } & \multirow{2}{*}{$\begin{array}{l}\text { Contributing } \\
\text { drainage area } \\
\qquad\left(\mathrm{mi}^{2}\right)\end{array}$} & \multicolumn{2}{|c|}{$\begin{array}{l}\text { Peak discharge (cubic feet per } \\
\text { second) }\end{array}$} \\
\hline & East & Wesxt & & East & West \\
\hline 0.1 & 440 & & 100 & 107,000 & 90,000 \\
\hline 0.15 & 785 & & 150 & 124,000 & 107,000 \\
\hline 0.2 & 1,140 & 690 & 200 & 136,000 & 120,000 \\
\hline 0.3 & 1,830 & 1,160 & 300 & 154,000 & 137,000 \\
\hline 0.4 & 2,520 & 1,640 & 400 & 168,000 & 149,000 \\
\hline 0.5 & 3,170 & 2,100 & 500 & 180,000 & 159,000 \\
\hline 0.6 & 3,820 & 2,500 & 600 & 191,000 & 167,000 \\
\hline 0.7 & 4,490 & 2,900 & 700 & 200,000 & 173,000 \\
\hline 0.8 & 5,080 & 3,280 & 800 & 208,000 & 178,000 \\
\hline 0.9 & 5,700 & 3,670 & 900 & 215,000 & 182,000 \\
\hline 1 & 6,300 & 4,020 & 1,000 & 222,000 & 187,000 \\
\hline 1.5 & 9,220 & 5,750 & 1,500 & 250,000 & 200,000 \\
\hline 2 & 12,100 & 7,300 & 2,000 & 272,000 & 211,000 \\
\hline 3 & 17,300 & 10,300 & 3,000 & 308,000 & 226,000 \\
\hline 4 & 21,900 & 12,900 & 4,000 & 335,000 & 235,000 \\
\hline 5 & 25,800 & 15,200 & 5,000 & 360,000 & 242,000 \\
\hline 6 & 29,100 & 17,400 & 6,000 & 379,000 & 248,000 \\
\hline 7 & 32,100 & 19,700 & 7,000 & 395,000 & 253,000 \\
\hline 8 & 34,700 & 21,700 & 8,000 & 411,000 & 257,000 \\
\hline 9 & 37,000 & 23,800 & 9,000 & 425,000 & 260,000 \\
\hline 10 & 39,500 & 25,500 & 10,000 & 440,000 & 264,000 \\
\hline 15 & 47,900 & 33,700 & 15,000 & 491,000 & 276,000 \\
\hline 20 & 54,800 & 41,000 & 20,000 & 529,000 & 286,000 \\
\hline 30 & 65,400 & 52,000 & 30,000 & 590,000 & 300,000 \\
\hline 40 & 74,100 & 60,000 & 40,000 & 637,000 & \\
\hline 50 & 82,000 & 66,500 & 50,000 & 672,000 & \\
\hline 60 & 88,000 & 72,100 & 60,000 & 705,000 & \\
\hline 70 & 93,500 & 77,800 & 70,000 & 735,000 & \\
\hline 80 & 98,800 & 82,000 & 80,000 & 760,000 & \\
\hline 90 & 102,500 & 86,500 & 90,000 & 785,000 & \\
\hline & & & 100,000 & 810,000 & \\
\hline & & & 150,000 & 900,000 & \\
\hline
\end{tabular}


of record within Oklahoma or about 25 kilometers into the bordering states of Arkansas, Kansas, Missouri, New Mexico, and Texas. These sites were in unregulated basins, and basins affected by regulation, urbanization, and irrigation.

Two types of documented extreme peak discharges are presented. These are maximum peak discharges documented at 352 sites with streamflow-gaging stations within and near Oklahoma and selected large peak discharges documented at 162 selected sites in Oklahoma at miscellaneous measurement sites without streamflow-gaging stations or streamflow-gaging stations with short record, with a total of 671 measurements. The sites are fairly well distributed statewide, however many streams, large and small, have never been monitored.

Potential extreme peak-discharge curves were developed for streamflow sites in hydrologic regions of the state based on documented extreme peak discharges and the contributing drainage areas. Two hydrologic regions, east and west, were defined, using 98 degrees 15 minutes longitude as the dividing line. The relation between the estimated 100-year flood frequency peak discharge and the contributing drainage area for each of the streamflow-gaging stations also was used to check and adjust each of the regional potential extreme peak-discharge curves.

A potential extreme peak-discharge estimate for any site in a unregulated basin can be obtained from the potential extreme peak-discharge curve for the hydrologic region containing the site, if the contributing drainage area is known. However, since all types of drainage basins were used to develop the curves, extreme peak-discharge estimates for sites in which there have been significant effects from manmade modification of streamflow may be obtained if caution is exercised to recognize the limitations of such estimates.

\section{Selected References}

Asquith, W.H., and Slade, R.M., Jr., 1995, Documented and potential extreme peak discharges and relation between potential extreme peak discharges and probable maximum flood peak discharges in Texas: U.S. Geological Survey Water-Resources Investigations Report 95-4249, 58 p.

, 1997, Regional equations for estimation of peak-streamflow frequency for natural basins in Texas: U.S. Geological Survey Water-Resources Investigations Report 96-4307, $68 \mathrm{p}$.

Bergman, D.L., and Huntzinger, T.L., 1981, Rainfall-runoff hydrographs and basin characteristics data for small streams in Oklahoma: U.S. Geological Survey Open-File Report 81$824,320 \mathrm{p}$.

Bergman, D.L., and Tortorelli, R.L., 1988, Flood of May 26-27, 1984, in Tulsa, Oklahoma: U.S. Geological Survey Hydrologic Investigations Atlas HA-707, 1 sheet.

Bingham, R.H., Bergman, D.L., and Thomas, W.O., Jr., 1974, Flood of October 1973 in Enid and vicinity, north-central
Oklahoma: U.S. Geological Survey Water-Resources Investigations Report 74-27, 2 sheets, scale 1:250,000, 1:126,720.

Bradshaw, H.A., 1945, Wewoka dam failure, April 14, 1945 : Oklahoma Planning and Resources Board, Oklahoma City, Division of Water Resources Report, 57 p.

Buckner, H.D., and Kurklin, J.K., 1984, Floods in south-central Oklahoma and north-central Texas: U.S. Geological Survey Open-File Report 84-065, 112 p.

Burnham, W.C., 1939, Washita River, Hammon flood, April 34, 1934: Oklahoma Planning and Resources Board, Oklahoma City, Division of Water Resources Report, 112 p.

Chow, V.T., Maidment, D.R., and Mays, L.W., 1988, Applied hydrology: New York, McGraw-Hill, 572 p.

Corley, R.K., and Huntzinger, T.L., 1979, Flood of August 2728, 1977, West Cache Creek and Blue Beaver Creek, southwestern Oklahoma: U.S. Geological Survey Open-File Report 79-276, 1 sheet, scale 1:24,00

Costa, J.E., 1987, A comparison of the largest rainfall-runoff floods in the United States with those of the People's Republic of China and the world: Journal of Hydrology, v. 96, no. 1-4, p. 101-115.

Creager, W.P., 1939, Possible and probable future floods: Civil Engineering, v. 9, p. 668-670.

Crippen, J.R., 1982, Envelope curves for extreme flood events: American Society of Civil Engineers, Proceedings of the 1982 Journal of Hydraulic Engineering, v. 108, p. 12081212.

Crippen, J.R., and Bue, C.D., 1977, Maximum floodflows in the conterminous United States: U.S. Geological Survey WaterSupply Paper 1887, $52 \mathrm{p}$.

Dalrymple, Tate, 1960, Flood-frequency analyses: U.S. Geological Survey Water-Supply Paper 1543-A, 80 p.

Hauth, L.D., 1985, Floods in central, southwest Oklahoma, October 17-23, 1983: U.S. Geological Survey Open-File Report 85-494, $21 \mathrm{p}$.

Heimann, D.C., and Tortorelli, R.L., 1988, Statistical summaries of streamflow records in Oklahoma and in parts of Arkansas, Kansas, Missouri, and Texas through 1984: U.S. Geological Survey Water-Resources Investigations Report 87-4205, 387 p.

Helsel, D.R., and Hirsch, R.M., 1992, Studies in Environmental Science 49, Statistical Methods in Water Resources: New York, Elsevier, 522 p.

Interagency Advisory Committee on Water Data (IACWD), 1982, Guidelines for determining flow frequency: Reston, Va., U.S. Geological Survey, Office of Water Data Coordination, Hydrology Subcommittee Bulletin 17B [variously paged].

Interagency Advisory Committee on Water Data (IACWD), 1986, Feasibility of assigning a probability to the probable maximum flood: Reston, Va., U.S. Geological Survey, Office of Water Data Coordination, $79 \mathrm{p}$.

Langbein, W.B., and Iseri, K.T., 1960, General introduction and hydrologic definitions: U.S. Geological Survey WaterSupply Paper 1541-A, 29 p. 
Leopold, L.B., and Maddock, Thomas, Jr., 1954, The flood control controversy: New York, Ronald Press Co., 278 p.

Matthai, H.F., 1969, Floods of June 1965 in South Platte River Basin, Colorado: U.S. Geological Survey Water-Supply Paper 1850-B, 64 p.

National Research Council, 1988, Estimating probabilities of extreme floods, methods and recommended research: Washington, D.C., National Academy Press, 141 p.

_ 1999 , Improving American river flood frequency analyses: Washington, D.C., National Academy Press, 132 p.

Patterson, J.L., 1964, Magnitude and frequency of floods in the United States, Part 7. Lower Mississippi River Basin: U.S. Geological Survey Water-Supply Paper 1681, 636 p.

Perry, C.A., Aldridge, B.N., and Ross, H.C., 2000, Summary of significant floods in the United States, Puerto Rico and the Virgin Islands, 1970 through 1989: U.S. Geological Survey Water-Supply Paper 2502, 598 p.

Sauer, V.B., 1974, Flood characteristics of Oklahoma streams: U.S. Geological Survey Water-Resources Investigations 5273, $301 \mathrm{p}$.

Thomas, B.E., Hjalmarson, H.W. and Waltemeyer, S.D., 1994, Methods for estimating magnitude and frequency of floods in the southwestern United States: U.S. Geological Survey Open-File Report 93-419, 211 p.

Thomas, W.O., Jr., and Corley, R.K., 1973, 1971-72 Floods on Glover Creek and Little River in southeastern Oklahoma: U.S. Geological Survey Water-Resources Investigations 573, 2 sheets, scale 1:24,000.

_ 1977 , Techniques for estimating flood discharges for Oklahoma streams: U.S. Geological Survey WaterResources Investigations Report 77-54, 170 p.

Tortorelli, R.L., 1996a, Estimated flood peak discharges on Twin, Brock, and Lightning Creeks, southwest Oklahoma City, Oklahoma, May 8, 1993: U.S. Geological Survey Water-Resources Investigations Report 96-4185, 127 p.

_ 1996b, Floods of April and May 1990 on the Arkansas, Red and Trinity Rivers in Oklahoma, Texas, Arkansas, and Louisiana, in U.S. Geological Survey, 1996, Summary of floods in the United States during 1990 and 1991: U.S. Geological Survey Water-Supply Paper 2474, p. 39-56.

-1997, Techniques for estimating peak-streamflow frequency for unregulated streams and streams regulated by small floodwater retarding structures in Oklahoma: U.S. Geological Survey Water-Resources Investigations Report 97-4202, 39 p.

Tortorelli, R.L., and Bergman, D.L., 1985. Techniques for estimating flood peak discharges for unregulated streams and streams regulated by small floodwater retarding structures in Oklahoma: U.S. Geological Survey Water-Resources Investigations Report 84-4358, 85 p.

Tortorelli, R.L., Cooter, E.J., and Schuelin, J.W., 1991,Oklahoma--Floods and droughts, in U.S. Geological Survey, 1991, National Water Summary 1988-1989: U.S. Geological Survey Water-Supply Paper 2375, p. 451-458.
U.S. Army Corps of Engineers, 1990, After action flood report, flood of April-May 1990 -- Southeastern Oklahoma, northeastern Texas: Tulsa District, 28 p.

U.S. Department of Agriculture, Soil Conservation Service, 1970, Storm Report, October 7, 8, 1970 (six sub-watersheds of Washita River) between Pauls Valley and Tishimingo, Oklahoma: $26 \mathrm{p}$.

U.S. Department of Commerce, 1958, Rainfall and floods of April, May, June 1957 in the south-central States: Weather Bureau Technical Paper 33, 350 p.

_ 1952, Kansas-Missouri floods of June-July 1951:

Weather Bureau Technical Paper 17, 105 p.

U.S. Geological Survey, 1954, Floods of May 1951 in western Oklahoma and northwestern Texas: U.S. Geological Survey Water-Supply Paper 1227-B, p. 135-199.

Wahl, K.L., and Tortorelli, R.L., 1997, Changes in flow in the Beaver-North Canadian River basin upstream from Canton Lake, western Oklahoma: Geological Survey WaterResources Investigations Report 96-4304, 58 p.

Walters, D.M., and Tortorelli, R.L., 1998, Oklahoma floods of May 8-14 and September 25-27, 1993, in U.S. Geological

Survey, 1998, Summary of Floods in the United States, January 1992 through September 1993: U.S. Geological Survey Water-Supply Paper 2499, p. 231-237.

Weiss, D.L., and Sullivan, C.L., 1958, Floods of April-May 1957 in Oklahoma and western Arkansas: U.S. Geological Survey Open-File Report 57-127, 21 p.

Westfall, A.O., and Patterson, J.L., 1964, Floods in Oklahoma, magnitude and frequency: U.S. Geological Survey Open-File Report 64-170, 105 p.

Wolman, M.G., and Costa, J.E., 1984, Envelope curves for extreme flood events--Discussion: American Society of Engineers, Proceedings of the 1984 Journal of Hydraulic Engineering, v. 110, p. 77-78.

Wright, J., 1990, April-May 1990 flood event at Bureau of Reclamation reservoirs (flood control): U.S. Bureau of Reclamation, Oklahoma-Texas Project Office Memorandum, 2 p. 


\section{Supplemental Information}


Table 1. Documented and potential extreme peak discharges and flood frequency estimates for selected streamflow-gaging stations with at least

[CONT, continuous record site; CSG, crest-stage partial record site; H, historic, I, irrigation; $\mathrm{N}$, unregulated; R, regulated; U, urban; ; $\mathrm{ft}^{3} / \mathrm{s}$, cubic feet per second Ck, creek; St, Street; blw, below; SWS, Subwatershed; Ave, Avenue; Lk, Lake; OKC, Oklahoma City; R., River; WY, water ye

\begin{tabular}{|c|c|c|c|c|c|c|c|}
\hline \multirow[b]{2}{*}{$\begin{array}{c}\text { Site } \\
\text { number } \\
\text { (fig. 1) }\end{array}$} & \multirow[b]{2}{*}{$\begin{array}{l}\text { Station } \\
\text { number }\end{array}$} & \multirow[b]{2}{*}{ Station name } & \multirow[b]{2}{*}{$\begin{array}{l}\text { Type of } \\
\text { station } \\
\text { (CONT/ } \\
\text { CSG) }\end{array}$} & \multicolumn{3}{|c|}{ Documented extreme peak discharge } & \multirow{2}{*}{ 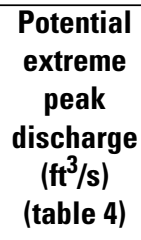 } \\
\hline & & & & $\begin{array}{l}\text { Type of } \\
\text { record (H/ } \\
\text { I/N// } \\
\text { R/U) }\end{array}$ & Date & $\begin{array}{c}\text { Discharge } \\
\left(\mathrm{ft}^{3} / \mathrm{s}\right)\end{array}$ & \\
\hline \multirow[t]{2}{*}{1} & 07146500 & Arkansas River at Arkansas City, Kans. & CONT & $\mathrm{N}$ & $06 / 10 / 23$ & 103,000 & 619,000 \\
\hline & & & & $\mathrm{R}$ & $11 / 03 / 98$ & 97,400 & \\
\hline \multirow[t]{2}{*}{2} & 07147800 & Walnut River at Winfield, Kans. & CONT & $\mathrm{N}$ & 04/23/44 & $105,000^{\mathrm{a}, \mathrm{b}}$ & 267,000 \\
\hline & & & & $\mathrm{R}$ & $11 / 02 / 98$ & 91,600 & \\
\hline 3 & 07148100 & Grouse Creek near Dexter, Kans. & CSG & $\mathrm{N}$ & $07 / 03 / 76$ & 51,000 & 129,000 \\
\hline 4 & 07148140 & Arkansas River near Ponca City, Okla. & CONT & $\mathrm{R}$ & $05 / 14 / 93$ & $62,900^{\mathrm{c}}$ & 632,000 \\
\hline \multirow[t]{2}{*}{5} & 07148350 & Salt Fork Arkansas River near Winchester, Okla. & CONT & $\mathrm{HN}$ & 05/00/57 & 80,000 & 180,000 \\
\hline & & & & $\mathrm{N}$ & 08/19/61 & 52,200 & \\
\hline \multirow[t]{2}{*}{6} & 07148400 & Salt Fork Arkansas River near Alva, Okla. & CONT & $\mathrm{N}$ & 10/23/41 & $27,000^{\mathrm{a}}$ & 187,000 \\
\hline & & & & $\mathrm{N}$ & $10 / 10 / 85$ & 12,800 & \\
\hline 7 & 07149000 & Medicine Lodge River near Kiowa, Kans. & CONT & $\mathrm{N}$ & 10/22/41 & $16,000^{\mathrm{a}}$ & 182,000 \\
\hline 8 & 07149500 & Salt Fork Arkansas River near Cherokee, Okla. & CONT & $\mathrm{N}$ & 10/23/41 & $35,000^{\mathrm{a}}$ & 218,000 \\
\hline \multirow[t]{2}{*}{9} & 07150500 & Salt Fork Arkansas River near Jet, Okla. & CONT & $\mathrm{N}$ & $05 / 19 / 38$ & 25,900 & 313,000 \\
\hline & & & & $\mathrm{R}$ & $04 / 02 / 73$ & 10,600 & \\
\hline 10 & 07150580 & Sand Creek Tributary near Kremlin, Okla. & CSG & $\mathrm{N}$ & $10 / 11 / 73$ & $12,000^{\mathrm{d}}$ & 32,600 \\
\hline 11 & 07150870 & Salt Fork Arkansas River Tributary near Eddy, Okla. & CSG & $\mathrm{N}$ & 09/06/69 & 1,320 & 13,900 \\
\hline \multirow{2}{*}{12} & 07151000 & Salt Fork of Arkansas River at Tonkawa, Okla. & CONT & $\mathrm{N}$ & 05/20/38 & $40,800^{\mathrm{a}}$ & 348,000 \\
\hline & & & & $\mathrm{R}$ & $10 / 11 / 73$ & $97,300^{\mathrm{d}}$ & \\
\hline \multirow[t]{2}{*}{13} & 07151500 & Chikaskia River near Corbin, Kans. & CONT & $\mathrm{HN}$ & 06/09/23 & $60,000^{\mathrm{a}}$ & 208,000 \\
\hline & & & & $\mathrm{N}$ & $10 / 11 / 85$ & 39,300 & \\
\hline \multirow[t]{2}{*}{14} & 07152000 & Chikaskia River near Blackwell, Okla. & CONT & HN & $06 / 10 / 23$ & $100,000^{\mathrm{a}}$ & 266,000 \\
\hline & & & & $\mathrm{N}$ & 06/22/42 & 85,000 & \\
\hline 15 & 07152360 & Elm Creek near Foraker, Okla. & CSG & $\mathrm{N}$ & $06 / 24 / 69$ & 9,200 & 52,300 \\
\hline 16 & 07152410 & Rock Creek near Shidler, Okla. & CSG & $\mathrm{N}$ & $05 / 18 / 65$ & 2,780 & 37,300 \\
\hline \multirow[t]{2}{*}{17} & 07152500 & Arkansas River at Ralston, Okla. & CONT & $\mathrm{N}$ & $10 / 13 / 73$ & $211,000^{d}$ & 661,000 \\
\hline & & & & $\mathrm{R}$ & $10 / 04 / 86$ & 174,000 & \\
\hline 18 & 07152520 & Black Bear Creek Tributary near Garber, Okla. & CSG & $\mathrm{N}$ & $08 / 14 / 74$ & 1,310 & 6,120 \\
\hline 19 & 07152842 & Subwatershed W-4 near Morrison, Okla. & CONT & $\mathrm{N}$ & $04 / 18 / 57$ & 496 & 1,970 \\
\hline 20 & 07152846 & Subwatershed W-3 near Morrison, Okla. & CONT & $\mathrm{N}$ & $07 / 15 / 51$ & 440 & 716 \\
\hline \multirow[t]{2}{*}{21} & 07153000 & Black Bear Creek at Pawnee, Okla. & CONT & $\mathrm{N}$ & $10 / 03 / 59$ & 30,200 & 188,000 \\
\hline & & & & $\mathrm{R}$ & $10 / 05 / 86$ & 19,200 & \\
\hline \multirow[t]{2}{*}{22} & 07153100 & Ranch Creek at Cleveland Dam, Okla. & CONT & $\mathrm{HN}$ & 09/04/40 & 32,400 & 56,800 \\
\hline & & & & $\mathrm{R}$ & $10 / 02 / 59$ & 11,800 & \\
\hline 23 & 07153500 & Dry Cimarron River near Guy, N. Mex. & CONT & $\mathrm{N}$ & $08 / 21 / 65$ & $46,100^{\mathrm{b}}$ & 163,000 \\
\hline 24 & 07154400 & Carrizozo Creek near Kenton, Okla. & CSG & $\mathrm{N}$ & 07/06/58 & 15,600 & 93,700 \\
\hline 25 & 07154500 & Cimarron River near Kenton, Okla. & CONT & $\mathrm{N}$ & $10 / 17 / 65$ & 43,400 & 188,000 \\
\hline 26 & 07154650 & Tesesquite Creek near Kenton, Okla. & CSG & $\mathrm{N}$ & 08/06/71 & 7,250 & 46,900 \\
\hline \multirow{2}{*}{$\begin{array}{l}26 \\
27 \\
27\end{array}$} & 07155000 & Cimarron River abv Ute Ck near Boise City, Okla. & CONT & $\mathrm{HN}$ & $04 / 20 / 42$ & $80,000^{a}$ & 208,000 \\
\hline & & & & $\mathrm{N}$ & 05/15/51 & $17,200^{\mathrm{e}}$ & \\
\hline 28 & 07155100 & Cold Springs Creek near Wheeless, Okla. & CSG & $\mathrm{N}$ & $08 / 21 / 65$ & 2,520 & 27,100 \\
\hline 29 & 07155590 & Cimarron River near Elkhart, Kans. & CONT & $\mathrm{N}$ & 05/26/77 & 21,500 & 217,000 \\
\hline \multirow[t]{2}{*}{30} & 07156900 & Cimarron River near Forgan, Okla. & CONT & $\mathrm{HN}$ & 00/00/42 & 69,000 & 237,000 \\
\hline & & & & $\mathrm{N}$ & 10/20/65 & 21,200 & \\
\hline 31 & 07157000 & Cimarron River near Mocane, Okla. & CONT & $\mathrm{N}$ & $05 / 17 / 51$ & $53,400^{\mathrm{e}}$ & 237,000 \\
\hline
\end{tabular}

8 years of annual peak-discharge data from unregulated, regulated, and urban basins within and near Oklahoma

mi² $^{2}$, square mile; E, sites east of 98 degrees 15 minutes longitude; W, sites west of 98 degrees 15 minutes longitude; LPIII, Log-Pearson Type III; abv, above;

\begin{tabular}{|c|c|c|c|c|c|c|c|c|c|c|c|c|}
\hline \multirow{2}{*}{$\begin{array}{l}\text { Site } \\
\text { number } \\
\text { (fig. 1) }\end{array}$} & \multirow{2}{*}{$\begin{array}{c}\text { Contrib- } \\
\text { uting } \\
\text { drainage } \\
\text { area }\left(\mathrm{mi}^{2}\right)\end{array}$} & \multirow{2}{*}{ Latitude } & \multirow{2}{*}{ Longitude } & \multirow{2}{*}{$\begin{array}{l}\text { Hydrologic } \\
\text { region } \\
(E / W)\end{array}$} & \multirow{2}{*}{$\begin{array}{c}\text { Type } \\
\text { basin } \\
\text { (N//R/R/ } \\
\text { U) }\end{array}$} & \multicolumn{7}{|c|}{$\begin{array}{l}\text { LPIII flood frequency estimates } \\
\text { Peak discharge for indicated recurrence interval }\left(\mathrm{ft}^{3} / \mathrm{s}\right)\end{array}$} \\
\hline & & & & & & $2 \mathrm{yr}$ & $5 \mathrm{yr}$ & $10 \mathrm{yr}$ & $25 \mathrm{yr}$ & $50 \mathrm{yr}$ & $100 \mathrm{yr}$ & $500 \mathrm{yr}$ \\
\hline \multirow[t]{2}{*}{1} & 36,106 & 0370323 & 0970332 & E & $\mathrm{N}$ & 14,900 & 31,000 & 44,600 & 65,000 & 82,200 & 101,000 & 152,000 \\
\hline & & & & & $\mathrm{R}$ & 22,900 & 44,100 & 61,200 & 86,000 & 106,000 & 128,000 & 186,000 \\
\hline \multirow[t]{2}{*}{2} & 1,880 & 0371327 & 0965940 & E & $\mathrm{N}$ & 18,100 & 34,000 & 46,700 & 65,100 & 80,200 & 96,500 & 139,000 \\
\hline & & & & & $\mathrm{R}$ & 19,100 & 38,100 & 54,300 & 78,600 & 99,500 & 123,000 & 186,000 \\
\hline 3 & 170 & 0371338 & 0964244 & E & $\mathrm{N}$ & 8,370 & 16,700 & 23,900 & 34,800 & 44,400 & 55,100 & 84,900 \\
\hline 4 & 38,923 & 0364136 & 0965548 & $\mathrm{E}$ & $\mathrm{R}$ & 18,200 & 29,000 & 37,200 & 48,400 & 57,400 & 67,000 & 91,800 \\
\hline 5 & 856 & 0365742 & 0984655 & w & $\mathrm{N}$ & 6,690 & 16,100 & 25,100 & 39,800 & 53,300 & 69,100 & 115,000 \\
\hline 6 & 1,009 & 0364854 & 0983852 & w & $\mathrm{N}$ & 7,200 & 15,100 & 21,600 & 30,800 & 38,400 & 46,400 & 66,700 \\
\hline 7 & 903 & 0370217 & 0982804 & w & $\mathrm{N}$ & 3,120 & 5,620 & 7,720 & 10,900 & 13,700 & 16,800 & 25,700 \\
\hline 8 & 2,439 & 0364906 & 0981908 & w & $\mathrm{N}$ & 13,600 & 23,700 & 31,500 & 42,500 & 51,400 & 61,000 & 85,600 \\
\hline 9 & 3,194 & 0364509 & 0980743 & E & $\mathrm{R}$ & 3,320 & 6,070 & 8,050 & 10,600 & 12,500 & 14,400 & 18,800 \\
\hline 10 & 7.21 & 0363300 & 0974838 & E & $\mathrm{N}$ & 384 & 731 & 1,050 & 1,580 & 2,070 & 2,670 & 4,560 \\
\hline 11 & 2.35 & 0364142 & 0972530 & E & $\mathrm{N}$ & 254 & 524 & 774 & 1,180 & 1,560 & 2,020 & 3,400 \\
\hline 12 & 4,520 & 0364019 & 0971833 & E & $\mathrm{R}$ & 13,000 & 25,700 & 36,600 & 53,000 & 67,200 & 83,000 & 127,000 \\
\hline 13 & 794 & 0370744 & 0973604 & E & $\mathrm{N}$ & 9,100 & 18,600 & 26,800 & 39,400 & 50,400 & 62,700 & 96,800 \\
\hline 14 & 1,859 & 0364841 & 0971637 & E & $\mathrm{N}$ & 18,700 & 38,000 & 55,200 & 82,200 & 106,000 & 134,000 & 215,000 \\
\hline 15 & 18.2 & 0365208 & 0963650 & E & $\mathrm{N}$ & 2,180 & 4,640 & 6,860 & 10,400 & 13,600 & 17,100 & 27,600 \\
\hline 16 & 9.13 & 0364450 & 0963730 & E & $\mathrm{N}$ & 1,630 & 2,090 & 2,380 & 2,730 & 2,990 & 3,230 & 3,780 \\
\hline \multirow[t]{2}{*}{17} & 46,850 & 0363015 & 0964341 & E & $\mathrm{N}$ & 56,900 & 110,000 & 152,000 & 211,000 & 259,000 & 310,000 & 438,000 \\
\hline & & & & & $\mathrm{R}$ & 47,600 & 87,200 & 117,000 & 158,000 & 190,000 & 223,000 & 303,000 \\
\hline 18 & 0.97 & 0362325 & 0973720 & E & $\mathrm{N}$ & 90 & 290 & 547 & 1,100 & 1,740 & 2,640 & 6,290 \\
\hline 19 & 0.32 & 0362107 & 0970402 & E & $\mathrm{N}$ & 132 & 228 & 303 & 409 & 495 & 587 & 827 \\
\hline 20 & 0.14 & 0362050 & 0970402 & E & $\mathrm{N}$ & 65 & 157 & 247 & 397 & 536 & 700 & 1,190 \\
\hline \multirow[t]{2}{*}{21} & 576 & 0362037 & 0964757 & E & $\mathrm{N}$ & 6,710 & 11,700 & 16,000 & 22,700 & 28,800 & 35,900 & 57,000 \\
\hline & & & & & $\mathrm{R}$ & 5,390 & 9,310 & 12,300 & 16,400 & 19,600 & 23,000 & 31,600 \\
\hline 22 & 21.9 & 0361700 & 0963435 & E & $\mathrm{R}$ & 1,480 & 3,800 & 5,840 & 8,860 & 11,300 & 13,900 & 20,300 \\
\hline 23 & 545 & 0365915 & 1032525 & W & $\mathrm{N}$ & 2,860 & 6,760 & 10,800 & 17,900 & 25,100 & 34,100 & 64,300 \\
\hline 24 & 111 & 0365255 & 1030105 & w & $\mathrm{N}$ & 1,720 & 4,440 & 7,170 & 11,800 & 16,100 & 21,300 & 36,800 \\
\hline 25 & 1,038 & 0365536 & 1025731 & w & $\mathrm{N}$ & 4,900 & 11,200 & 17,200 & 27,400 & 37,000 & 48,400 & 83,600 \\
\hline 26 & 25.4 & 0365352 & 1025404 & W & $\mathrm{N}$ & 1,400 & 4,050 & 6,780 & 11,400 & 15,700 & 20,800 & 35,600 \\
\hline 27 & 1,879 & 0365446 & 1023708 & W & $\mathrm{N}$ & 8,600 & 16,000 & 21,800 & 30,100 & 36,800 & 43,900 & 62,000 \\
\hline 28 & 11.0 & 0364620 & 1024816 & W & $\mathrm{N}$ & 89 & 419 & 938 & 2,200 & 3,800 & 6,200 & 16,600 \\
\hline 29 & 2,406 & 0370730 & 1015350 & w & $\mathrm{N}$ & 1,290 & 4,110 & 7,280 & 13,000 & 18,600 & 25,600 & 47,000 \\
\hline 30 & 4,220 & 0370040 & 1002929 & w & $\mathrm{N}$ & 861 & 3,130 & 6,160 & 12,700 & 20,300 & 31,000 & 73,300 \\
\hline 31 & 4,305 & 0365833 & 1001850 & w & $\mathrm{N}$ & 5,210 & 11,800 & 18,600 & 30,900 & 43,300 & 59,200 & 114,000 \\
\hline
\end{tabular}




\begin{tabular}{|c|c|c|c|c|c|c|c|}
\hline \multirow[b]{2}{*}{$\begin{array}{c}\text { Site } \\
\text { number } \\
\text { (fig. 1) }\end{array}$} & \multirow[b]{2}{*}{$\begin{array}{l}\text { Station } \\
\text { number }\end{array}$} & \multirow[b]{2}{*}{ Station name } & \multirow[b]{2}{*}{$\begin{array}{l}\text { Type of } \\
\text { station } \\
\text { (CONT/ } \\
\text { CSG) }\end{array}$} & \multicolumn{3}{|c|}{ Documented extreme peak discharge } & \multirow[b]{2}{*}{$\begin{array}{c}\text { Potential } \\
\text { extreme } \\
\text { peak } \\
\text { discharge } \\
\left.\text { (ft' } \text { frs }^{3}\right) \\
\text { (table 4) }\end{array}$} \\
\hline & & & & $\begin{array}{l}\text { Type of } \\
\text { record (H/ } \\
\text { //N// } \\
\text { R/U) }\end{array}$ & Date & $\begin{array}{c}\text { Discharge } \\
\left(\mathrm{ft}^{3} / \mathbf{s}\right)\end{array}$ & \\
\hline 32 & 07157500 & Crooked Creek near Englewood, Kans. & CONT & $\mathrm{N}$ & $05 / 20 / 55$ & $13,600^{\mathrm{a}}$ & 179,000 \\
\hline 33 & 07157550 & West Fork Creek near Knowles, Okla. & CSG & $\mathrm{N}$ & $08 / 14 / 67$ & 1,150 & 13,400 \\
\hline 34 & 07157700 & Keiger Creek near Ashland, Kans. & CSG & $\mathrm{N}$ & $07 / 21 / 61$ & 1,250 & 55,200 \\
\hline 35 & 07157950 & Cimarron River near Buffalo, Okla. & CONT & $\mathrm{N}$ & $09 / 26 / 73$ & 26,400 & 254,000 \\
\hline 36 & 07157960 & Buffalo Creek near Lovedale, Okla. & CONT & $\mathrm{N}$ & $08 / 09 / 67$ & 15,800 & 150,000 \\
\hline 37 & 07158000 & Cimarron River near Waynoka, Okla. & CONT & $\mathrm{N}$ & $05 / 16 / 57$ & $94,500^{\mathrm{a}}$ & 259,000 \\
\hline 38 & 07158020 & Cimarron River Tributary near Lone Wolf, Okla. & CSG & $\mathrm{N}$ & $11 / 02 / 74$ & 921 & 13,500 \\
\hline \multirow[t]{2}{*}{39} & 07158080 & Sand Creek Tributary near Waynoka, Okla. & CSG & HN & $07 / 04 / 51$ & 990 & 6,090 \\
\hline & & & & $\mathrm{N}$ & $08 / 21 / 70$ & 587 & \\
\hline 40 & 07158120 & Cimarron River Tributary near Isabella, Okla. & CSG & $\mathrm{N}$ & $05 / 07 / 69$ & 207 & 2,580 \\
\hline 41 & 07158180 & Salt Creek Tributary near Okeene, Okla. & CSG & $\mathrm{N}$ & 09/20/74 & 4,500 & 22,200 \\
\hline 42 & 07158400 & Salt Creek near Okeene, Okla. & CONT & $\mathrm{N}$ & $09 / 19 / 74$ & 12,700 & 135,000 \\
\hline 43 & 07158500 & Preacher Creek near Dover, Okla. & CSG & $\mathrm{N}$ & $05 / 15 / 57$ & $6,420^{\mathrm{a}}$ & 47,100 \\
\hline 44 & 07158550 & Turkey Creek Tributary near Goltry, Okla. & CSG & $\mathrm{N}$ & $05 / 26 / 76$ & 5,050 & 26,100 \\
\hline \multirow[t]{2}{*}{45} & 07159000 & Turkey Creek near Drummond, Okla. ${ }^{1}$ & CSG & HN & $00 / 00 / 32$ & 30,000 & 145,000 \\
\hline & & & & $\mathrm{N}$ & $10 / 11 / 73$ & $36,300^{\mathrm{d}}$ & \\
\hline 46 & 07159100 & Cimarron River near Dover, Okla. & CONT & $\mathrm{N}$ & 10/03/86 & 123,000 & 448,000 \\
\hline \multirow{2}{*}{47} & 07159200 & Kingfisher Creek near Kingfisher, Okla. ${ }^{1}$ & CSG & HN & $06 / 23 / 48$ & $55,000^{\mathrm{a}}$ & 126,000 \\
\hline & & & & $\mathrm{N}$ & 05/27/77 & 20,700 & \\
\hline 48 & 07159750 & Cottonwood Creek near Seward, Okla. & CONT & $\mathrm{R}$ & 06/09/95 & 43,500 & 157,000 \\
\hline 49 & 07159810 & Watershed W-IV near Guthrie, Okla. & CONT & $\mathrm{N}$ & $00 / 00 / 49$ & 271 & 716 \\
\hline \multirow{2}{*}{50} & 07160000 & Cimarron River near Guthrie, Okla. & CONT & HN & $05 / 00 / 35$ & 90,000 & 460,000 \\
\hline & & & & $\mathrm{N}$ & $05 / 17 / 57$ & $158,000^{\mathrm{a}}$ & \\
\hline 51 & 07160500 & Skeleton Creek near Lovell, Okla. & CONT & $\mathrm{N}$ & $05 / 16 / 57$ & $75,200^{\mathrm{a}, \mathrm{b}}$ & 169,000 \\
\hline 52 & 07160550 & West Beaver Creek near Orlando, Okla. & CSG & $\mathrm{N}$ & $05 / 07 / 82$ & 4,400 & 46,100 \\
\hline 53 & 07161000 & Cimarron River at Perkins, Okla. & CONT & $\mathrm{N}$ & 10/04/86 & 162,000 & 470,000 \\
\hline 54 & 07161450 & Cimarron River near Ripley, Okla. ${ }^{2}$ & CONT & $\mathrm{N}$ & $05 / 10 / 93$ & $141,000^{c}$ & 471,000 \\
\hline \multirow[t]{2}{*}{55} & 07163000 & Council Creek near Stillwater, Okla. & CONT & $\mathrm{HN}$ & $04 / 27 / 12$ & 14,400 & 66,300 \\
\hline & & & & $\mathrm{N}$ & 10/02/59 & $25,000^{\mathrm{b}}$ & \\
\hline 56 & 07163020 & Corral Creek near Yale, Okla. & CSG & $\mathrm{N}$ & $09 / 21 / 65$ & 1,260 & 16,700 \\
\hline 57 & 07163500 & Cimarron River at Oilton, Okla. & CONT & $\mathrm{N}$ & $06 / 21 / 35$ & 72,300 & 478,000 \\
\hline 58 & 07164000 & Cimarron River at Mannford, Okla. & CONT & $\mathrm{N}$ & $05 / 18 / 57$ & 145,000 & 478,000 \\
\hline \multirow[t]{3}{*}{59} & 07164500 & Arkansas River at Tulsa, Okla. & CONT & HN & $06 / 13 / 23$ & 244,000 & 711,000 \\
\hline & & & & $\mathrm{N}$ & $10 / 05 / 59$ & 246,000 & \\
\hline & & & & & 10/05/86 & 307,000 & \\
\hline 60 & 07164600 & Joe Creek at 61st Street at Tulsa, Okla. & CONT & $\mathrm{U}$ & 06/09/95 & 11,100 & 43,200 \\
\hline \multirow[t]{3}{*}{61} & 07165500 & Polecat Creek below Heyburn Reservoir & CONT & HN & $09 / 04 / 40$ & $26,000^{\mathrm{a}}$ & 115,000 \\
\hline & & near Heyburn, Okla. & & $\mathrm{N}$ & $05 / 19 / 49$ & 17,300 & \\
\hline & & & & $\mathrm{R}$ & $11 / 04 / 74$ & 2,080 & \\
\hline 62 & 07165550 & Snake Creek near Bixby, Okla. ${ }^{1}$ & CSG & $\mathrm{N}$ & 06/09/74 & 9,280 & 82,000 \\
\hline 63 & 07165562 & Haikey Ck at 101 st St South at Tulsa, Okla. & CONT & $\mathrm{U}$ & 10/05/98 & 6,910 & 51,800 \\
\hline 64 & 07165565 & Little Haikey Ck at 101 st St South at Tulsa, Okla. & CONT & $\mathrm{U}$ & 10/05/98 & 2,310 & 27,300 \\
\hline 65 & 07165570 & Arkansas River near Haskell, Okla. & CONT & $\mathrm{R}$ & 10/05/86 & 259,000 & 714,000 \\
\hline 66 & 07170500 & Verdigris River at Independence, Kans. & CONT & $\mathrm{N}$ & $04 / 17 / 45$ & $117,000^{\mathrm{a}}$ & 304,000 \\
\hline
\end{tabular}

\begin{tabular}{|c|c|c|c|c|c|c|c|c|c|c|c|c|}
\hline \multirow{2}{*}{$\begin{array}{l}\text { Site } \\
\text { number } \\
\text { (fig. 1) }\end{array}$} & \multirow{2}{*}{$\begin{array}{c}\text { Contrib- } \\
\text { uting } \\
\text { drainage } \\
\text { area }\left(\mathrm{mi}^{2}\right)\end{array}$} & \multirow{2}{*}{ Latitude } & \multirow{2}{*}{ Longitude } & \multirow{2}{*}{$\begin{array}{l}\text { Hydrologic } \\
\text { region (E/ } \\
\text { W) }\end{array}$} & \multirow{2}{*}{$\begin{array}{c}\text { Type } \\
\text { basin } \\
\text { (N///R/ } \\
\text { U) }\end{array}$} & \multicolumn{7}{|c|}{$\begin{array}{l}\text { LPIII flood frequency estimates } \\
\text { Peak discharge for indicated recurrence interval }\left(\mathrm{ft}^{3} / \mathrm{s}\right)\end{array}$} \\
\hline & & & & & & $2 \mathrm{yr}$ & $5 \mathrm{yr}$ & $10 \mathrm{yr}$ & $25 \mathrm{yr}$ & $50 \mathrm{yr}$ & $100 \mathrm{yr}$ & $500 \mathrm{yr}$ \\
\hline 32 & 813 & 0370154 & 1001229 & $\mathrm{~W}$ & $\mathrm{~N}$ & 902 & 3,440 & 6,520 & 12,400 & 18,300 & 25,600 & 48,600 \\
\hline 33 & 4.22 & 0365230 & 1000720 & $\mathrm{w}$ & $\mathrm{N}$ & 106 & 271 & 435 & 713 & 974 & 1,280 & 2,220 \\
\hline 34 & 34.0 & 0371136 & 0995448 & w & $\mathrm{N}$ & 391 & 686 & 905 & 1,200 & 1,440 & 1,680 & 2,270 \\
\hline 35 & 7,191 & 0365107 & 0991854 & w & $\mathrm{N}$ & 3,410 & 8,480 & 13,100 & 20,100 & 26,200 & 32,700 & 50,000 \\
\hline 36 & 408 & 0364614 & 0992200 & w & $\mathrm{N}$ & 1,050 & 4,110 & 7,980 & 15,700 & 23,800 & 34,200 & 68,800 \\
\hline 37 & 8,504 & 0363102 & 0985245 & w & $\mathrm{N}$ & 14,400 & 32,400 & 46,800 & 66,600 & 82,000 & 97,700 & 134,000 \\
\hline 38 & 4.26 & 0362425 & 0984410 & w & $\mathrm{N}$ & 534 & 771 & 929 & 1,130 & 1,280 & 1,420 & 1,770 \\
\hline 39 & 1.61 & 0363540 & 0984400 & w & $\mathrm{N}$ & 146 & 360 & 570 & 923 & 1,260 & 1,650 & 2,850 \\
\hline 40 & 0.62 & 0361630 & 0982100 & W & $\mathrm{N}$ & 83 & 143 & 190 & 255 & 308 & 365 & 513 \\
\hline 41 & 8.23 & 0360300 & 0981900 & $\mathrm{w}$ & $\mathrm{N}$ & 660 & 1,960 & 3,500 & 6,540 & 9,810 & 14,200 & 30,100 \\
\hline 42 & 196 & 0360611 & 0981136 & E & $\mathrm{N}$ & 4,590 & 7,130 & 9,060 & 11,800 & 14,000 & 16,500 & 22,900 \\
\hline 43 & 14.5 & 0360230 & 0980048 & E & $\mathrm{N}$ & 200 & 521 & 897 & 1,640 & 2,440 & 3,520 & 7,600 \\
\hline 44 & 5.08 & 0362840 & 0980805 & E & $\mathrm{N}$ & 342 & 999 & 1,760 & 3,230 & 4,790 & 6,840 & 14,100 \\
\hline 45 & 248 & 0361905 & 0980003 & E & $\mathrm{N}$ & 2,630 & 7,200 & 12,200 & 21,500 & 31,100 & 43,300 & 85,000 \\
\hline 46 & 10,787 & 0355706 & 0975451 & E & $\mathrm{N}$ & 26,700 & 51,200 & 71,700 & 102,000 & 128,000 & 157,000 & 237,000 \\
\hline 47 & 157 & 0355003 & 0980357 & E & $\mathrm{N}$ & 3,070 & 9,820 & 18,300 & 36,000 & 55,800 & 83,400 & 190,000 \\
\hline 48 & 320 & 0354849 & 0972840 & E & $\mathrm{R}$ & 8,220 & 19,800 & 30,400 & 46,800 & 61,000 & 76,800 & 119,000 \\
\hline 49 & 0.14 & 0354847 & 0972414 & E & $\mathrm{N}$ & 30 & 80 & 137 & 250 & 371 & 534 & 1,140 \\
\hline 50 & 11,966 & 0355514 & 0972532 & E & $\mathrm{N}$ & 30,200 & 58,000 & 78,600 & 106,000 & 127,000 & 147,000 & 196,000 \\
\hline 51 & 410 & 0360336 & 0973505 & E & $\mathrm{N}$ & 5,320 & 14,200 & 24,400 & 43,900 & 64,900 & 92,800 & 195,000 \\
\hline 52 & 13.9 & 0360845 & 0972805 & E & $\mathrm{N}$ & 972 & 2,190 & 3,380 & 5,400 & 7,330 & 9,680 & 17,100 \\
\hline 53 & 12,926 & 0355727 & 0970154 & E & $\mathrm{N}$ & 31,200 & 61,800 & 86,200 & 121,000 & 149,000 & 178,000 & 252,000 \\
\hline 54 & 13,053 & 0355909 & 0965443 & E & $\mathrm{N}$ & 33,200 & 65,500 & 90,800 & 126,000 & 154,000 & 183,000 & 254,000 \\
\hline 55 & 31.0 & 0360658 & 0965203 & E & $\mathrm{N}$ & 2,150 & 4,660 & 7,190 & 11,700 & 16,200 & 21,900 & 41,500 \\
\hline 56 & 2.89 & 0360750 & 0964950 & E & $\mathrm{N}$ & 582 & 908 & 1,160 & 1,530 & 1,850 & 2,190 & 3,150 \\
\hline 57 & 13,743 & 0360538 & 0963452 & E & $\mathrm{N}$ & 37,500 & 50,400 & 58,600 & 68,700 & 76,000 & 83,200 & 99,400 \\
\hline 58 & 13,923 & 0360932 & 0962354 & E & $\mathrm{N}$ & 33,100 & 61,000 & 82,300 & 112,000 & 135,000 & 160,000 & 220,000 \\
\hline 59 & 62,074 & 0360826 & 0960022 & E & $\mathrm{N}$ & 80,000 & 140,000 & 183,000 & 239,000 & 282,000 & 324,000 & 422,000 \\
\hline & & & & & $\mathrm{R}$ & 42,900 & 82,800 & 117,000 & 169,000 & 215,000 & 266,000 & 413,000 \\
\hline 60 & 12.2 & 0360432 & 0955737 & E & $\mathrm{U}$ & 5,750 & 8,020 & 9,570 & 11,600 & 13,100 & 14,700 & 18,500 \\
\hline 61 & 123 & 0355642 & 0961739 & E & $\mathrm{N}$ & 8,820 & 16,900 & 24,400 & 36,600 & 48,100 & 61,900 & 105,000 \\
\hline & & & & & $\mathrm{R}$ & 1,390 & 1,890 & 2,160 & 2,450 & 2,630 & 2,780 & 3,080 \\
\hline 62 & 50.0 & 0354908 & 0955318 & E & $\mathrm{N}$ & & & 7,930 & 11,200 & 14,100 & 17,400 & 26,900 \\
\hline 63 & 17.8 & 0360101 & 0955055 & E & U & & 4,990 & 6,420 & 8,380 & 9,940 & 11,600 & 15,700 \\
\hline 64 & 5.45 & 0360103 & 0955138 & E & $\mathrm{U}$ & 1,080 & 1,560 & 1,910 & 2,400 & 2,800 & 3,220 & 4,330 \\
\hline 65 & 62,932 & 0354915 & 0953819 & E & $\mathrm{R}$ & 52,400 & 93,600 & 129,000 & 185,000 & 236,000 & 295,000 & 471,000 \\
\hline 6 & 2,892 & 0371326 & 0954043 & $\mathrm{E}$ & $\mathrm{N}$ & 28,100 & 49,300 & 66,100 & 90,200 & 110,000 & 132,000 & 190,000 \\
\hline
\end{tabular}




\begin{tabular}{|c|c|c|c|c|c|c|c|}
\hline \multirow[b]{2}{*}{$\begin{array}{c}\text { Site } \\
\text { number } \\
\text { (fig. 1) }\end{array}$} & \multirow[b]{2}{*}{$\begin{array}{l}\text { Station } \\
\text { number }\end{array}$} & \multirow[b]{2}{*}{ Station name } & \multirow[b]{2}{*}{$\begin{array}{l}\text { Type of } \\
\text { station } \\
\text { (CONT/ } \\
\text { CSG) }\end{array}$} & \multicolumn{3}{|c|}{ Documented extreme peak discharge } & \multirow[b]{2}{*}{$\begin{array}{c}\text { Potential } \\
\text { extreme } \\
\text { peak } \\
\text { discharge } \\
\left(\mathrm{ft}^{3} / \mathrm{s}\right) \\
(\text { table 4) }\end{array}$} \\
\hline & & & & $\begin{array}{c}\text { Type of } \\
\text { record (H/ } \\
\text { 1/N/ } \\
\text { R/U) }\end{array}$ & Date & $\begin{array}{c}\text { Discharge } \\
\left(\mathrm{ft}^{3} / \mathbf{s}\right)\end{array}$ & \\
\hline 66 & & & & $\mathrm{R}$ & $10 / 04 / 86$ & 109,000 & \\
\hline 67 & 07170800 & Mud Creek near Mound City, Kans. & CSG & $\mathrm{N}$ & $07 / 03 / 76$ & 7,500 & 22,800 \\
\hline \multirow[t]{2}{*}{68} & 07171000 & Verdigris River near Lenapah, Okla. & CONT & $\mathrm{N}$ & $05 / 20 / 43$ & $137,000^{\text {a }}$ & 325,000 \\
\hline & & & & $\mathrm{R}$ & 10/05/86 & 81,500 & \\
\hline 69 & 07171120 & Clear Creek Tributary near Hollow, Okla. & CSG & $\mathrm{N}$ & $03 / 08 / 74$ & 1,040 & 13,100 \\
\hline 70 & 07171400 & Verdigris River near Oologah, Okla. & CONT & $\mathrm{R}$ & $10 / 14 / 86$ & 53,700 & 343,000 \\
\hline 71 & 07171700 & Spring Branch near Cedar Vale, Kans. & CSG & $\mathrm{N}$ & 10/02/86 & 3,650 & 17,800 \\
\hline 72 & 07171800 & Cedar Creek Tributary near Hooser, Kans. & CSG & $\mathrm{N}$ & $10 / 03 / 86$ & 720 & 3,560 \\
\hline \multirow{2}{*}{73} & 07171900 & Grant Creek near Wauneta, Kans. & CSG & $\mathrm{N}$ & 09/13/61 & 9,000 & 54,800 \\
\hline & & & & $\mathrm{R}$ & $06 / 22 / 77$ & 6,000 & \\
\hline \multirow[t]{2}{*}{74} & 07172000 & Caney River near Elgin, Kans. & CONT & $\mathrm{N}$ & $09 / 13 / 61$ & $62,000^{\mathrm{b}}$ & 173,000 \\
\hline & & & & $\mathrm{R}$ & 10/03/86 & 104,000 & \\
\hline \multirow[t]{2}{*}{75} & 07173000 & Caney River near Hulah, Okla. & CONT & $\mathrm{N}$ & $04 / 10 / 44$ & $51,000^{\mathrm{a}}$ & 203,000 \\
\hline & & & & $\mathrm{R}$ & $10 / 03 / 86$ & 58,000 & \\
\hline 76 & 07174000 & Little Caney River near Copan, Okla. & CONT & $\mathrm{N}$ & $04 / 10 / 44$ & $36,400^{\mathrm{a}}$ & 171,000 \\
\hline \multirow{2}{*}{77} & 07174200 & Little Caney River blw Cotton Ck, near Copan, Okla. ${ }^{3}$ & CONT & HN & $04 / 00 / 44$ & 43,100 & 180,000 \\
\hline & & & & $\mathrm{R}$ & $03 / 10 / 74$ & 33,200 & \\
\hline 78 & 07174400 & Caney River abv Coon Creek at Bartlesville, Okla. & CONT & $\mathrm{R}$ & 10/04/86 & 94,500 & 244,000 \\
\hline 79 & 07174570 & Dry Hollow near Pawhuska, Okla. & CSG & $\mathrm{N}$ & $07 / 14 / 65$ & 660 & 10,200 \\
\hline 80 & 07174600 & Sand Creek at Okesa, Okla. & CONT & $\mathrm{N}$ & 05/09/93 & $20,200^{\mathrm{c}}$ & 120,000 \\
\hline 81 & 07174700 & Caney River near Ochelata, Okla. & CONT & $\mathrm{N}$ & $06 / 13 / 57$ & 33,800 & 261,000 \\
\hline 82 & 07174720 & Hogshooter Creek Tributary near Bartlesville, Okla. & CSG & $\mathrm{N}$ & $06 / 24 / 69$ & 919 & 5,940 \\
\hline 83 & 07175000 & Double Creek SWS 5 near Ramona, Okla. & CONT & $\mathrm{R}$ & $06 / 23 / 57$ & $3,580^{\mathrm{b}}$ & 14,100 \\
\hline \multirow[t]{2}{*}{84} & 07175500 & Caney River near Ramona, Okla. & CONT & $\mathrm{N}$ & $10 / 03 / 45$ & $38,500^{\mathrm{a}}$ & 270,000 \\
\hline & & & & $\mathrm{R}$ & $10 / 05 / 86$ & 85,600 & \\
\hline \multirow[t]{2}{*}{85} & 07176000 & Verdigris River near Claremore, Okla. & CONT & $\mathrm{N}$ & $05 / 21 / 43$ & $182,000^{\mathrm{a}}$ & 388,000 \\
\hline & & & & $\mathrm{R}$ & 10/12/86 & 78,400 & \\
\hline 86 & 07176465 & Birch Creek blw Birch Lake near Barnsdall, Okla. & CONT & $\mathrm{R}$ & 10/07/86 & 2,070 & 91,300 \\
\hline \multirow[t]{2}{*}{87} & 07176500 & Bird Creek at Avant, Okla. & CONT & $\mathrm{N}$ & 10/02/59 & 32,400 & 163,000 \\
\hline & & & & $\mathrm{R}$ & $06 / 10 / 85$ & 27,900 & \\
\hline 88 & 07176800 & Candy Creek near Wolco, Okla. & CONT & $\mathrm{N}$ & $03 / 10 / 74$ & 9,520 & 65,900 \\
\hline 89 & 07177000 & Hominy Creek near Skiatook, Okla. & CONT & $\mathrm{N}$ & 10/03/59 & 35,600 & 160,000 \\
\hline \multirow[t]{2}{*}{90} & 07177500 & Bird Creek near Sperry, Okla. & CONT & $\mathrm{N}$ & 10/03/59 & 90,000 & 215,000 \\
\hline & & & & $\mathrm{R}$ & $05 / 10 / 93$ & $30,600^{\mathrm{c}}$ & \\
\hline 91 & 07177650 & Flat Rock Creek at Cincinnati Ave at Tulsa, Okla. & CONT & $\mathrm{U}$ & 05/04/99 & 4,580 & 35,200 \\
\hline 92 & 07177800 & Coal Creek at Tulsa, Okla. & CONT & $\mathrm{U}$ & $06 / 23 / 95$ & 5,190 & 33,500 \\
\hline 93 & 07178000 & Bird Creek near Owasso, Okla. & CONT & $\mathrm{R}$ & $05 / 11 / 93$ & 29,200 & 223,000 \\
\hline \multirow[t]{2}{*}{94} & 07178040 & Mingo Creek at 46th Street North at Tulsa, Okla. & CONT & $\mathrm{HU}$ & $05 / 27 / 84$ & $47,500^{\mathrm{f}}$ & 88,000 \\
\hline & & & & $\mathrm{U}$ & $08 / 20 / 89$ & 9,920 & \\
\hline 95 & 07178200 & Bird Creek at State Highway 266 near Catoosa, Okla. & CONT & $\mathrm{R}$ & $05 / 11 / 93$ & $27,400^{\mathrm{c}}$ & 228,000 \\
\hline \multirow[t]{3}{*}{96} & 07178600 & Verdigris River near Inola, Okla. & CONT & HN & $05 / 21 / 43$ & $224,000^{\mathrm{a}}$ & 410,000 \\
\hline & & & & $\mathrm{N}$ & $05 / 12 / 61$ & 118,000 & \\
\hline & & & & $\mathrm{R}$ & $05 / 01 / 70$ & 39,600 & \\
\hline 97 & 07178640 & Bull Creek near Inola, Okla. & CSG & $\mathrm{N}$ & $06 / 03 / 73$ & 1,570 & 41,300 \\
\hline 98 & 07184500 & Labette Creek near Oswego, Kans. & CSG & HN & 05/00/35 & 21,000 & 138,000 \\
\hline
\end{tabular}

\begin{tabular}{|c|c|c|c|c|c|c|c|c|c|c|c|c|}
\hline \multirow{2}{*}{$\begin{array}{c}\text { Site } \\
\text { number } \\
\text { (fig. 1) }\end{array}$} & \multirow{2}{*}{$\begin{array}{c}\text { Contrib- } \\
\text { uting } \\
\text { drainage } \\
\text { area }\left(\mathrm{mi}^{2}\right)\end{array}$} & \multirow{2}{*}{ Latitude } & \multirow{2}{*}{ Longitude } & \multirow{2}{*}{$\begin{array}{l}\text { Hydrologic } \\
\text { region (E/ } \\
\text { W) }\end{array}$} & \multirow{2}{*}{$\begin{array}{c}\text { Type } \\
\text { basin } \\
\text { (N///R/ } \\
\text { U) }\end{array}$} & \multicolumn{7}{|c|}{$\begin{array}{l}\text { LPIII flood frequency estimates } \\
\text { Peak discharge for indicated recurrence interval }\left(\mathrm{ft}^{3} / \mathrm{s}\right)\end{array}$} \\
\hline & & & & & & $2 \mathrm{yr}$ & $5 \mathrm{yr}$ & $10 \mathrm{yr}$ & $25 \mathrm{yr}$ & $50 \mathrm{yr}$ & $100 \mathrm{yr}$ & $500 \mathrm{yr}$ \\
\hline 66 & & & & & $\mathrm{R}$ & 22,000 & 34,900 & 45,200 & 60,100 & 72,700 & 86,800 & 126,000 \\
\hline 67 & 4.22 & 0371138 & 0952652 & E & $\mathrm{N}$ & 1,270 & 2,180 & 2,880 & 3,850 & 4,640 & 5,480 & 7,650 \\
\hline \multirow[t]{2}{*}{68} & 3,639 & 0365104 & 0953509 & E & $\mathrm{N}$ & 33,800 & 58,000 & 77,800 & 107,000 & 132,000 & 161,000 & 240,000 \\
\hline & & & & & $\mathrm{R}$ & 32,400 & 47,500 & 58,100 & 72,000 & 82,800 & 93,900 & 121,000 \\
\hline 69 & 2.19 & 0365250 & 0951600 & E & $\mathrm{N}$ & 423 & 613 & 748 & 925 & 1,060 & 1,210 & 1,560 \\
\hline 70 & 4,339 & 0362514 & 0954103 & E & $\mathrm{R}$ & 20,500 & 27,900 & 32,600 & 38,300 & 42,300 & 46,200 & 55,000 \\
\hline 71 & 3.10 & 0370648 & 0962729 & E & $\mathrm{N}$ & 840 & 2,160 & 3,310 & 4,960 & 6,280 & 7,630 & 10,800 \\
\hline 72 & 0.56 & 0370627 & 0963427 & E & $\mathrm{N}$ & 148 & 334 & 488 & 706 & 880 & 1,060 & 1,490 \\
\hline 73 & 20.0 & 0370634 & 0962355 & E & $\mathrm{R}$ & 2,570 & 3,980 & 4,960 & 6,210 & 7,150 & 8,090 & 10,300 \\
\hline \multirow[t]{2}{*}{74} & 445 & 0370013 & 0961854 & E & $\mathrm{N}$ & 13,900 & 28,400 & 38,800 & 52,100 & 61,600 & 70,600 & 89,800 \\
\hline & & & & & $\mathrm{R}$ & 16,100 & 29,000 & 38,500 & 51,100 & 60,700 & 70,400 & 93,300 \\
\hline \multirow[t]{2}{*}{75} & 733 & 0365537 & 0960506 & E & $\mathrm{N}$ & 14,900 & 25,600 & 32,900 & 42,100 & 48,800 & 55,300 & 69,700 \\
\hline & & & & & $\mathrm{R}$ & 3,540 & 6,830 & 10,200 & 16,200 & 22,400 & 30,500 & 59,800 \\
\hline 76 & 424 & 0365815 & 0955605 & E & $\mathrm{N}$ & 10,900 & 20,100 & 26,800 & 35,400 & 42,000 & 48,400 & 63,100 \\
\hline \multirow[t]{2}{*}{77} & 502 & 0365342 & 0955809 & E & $\mathrm{N}$ & 12,700 & 20,400 & 25,800 & 33,000 & 38,600 & 44,200 & 57,900 \\
\hline & & & & & $\mathrm{R}$ & 6,740 & 12,500 & 18,100 & 27,800 & 37,300 & 49,400 & 90,600 \\
\hline 78 & 1,392 & 0364520 & 0955819 & E & $\mathrm{R}$ & 8,720 & 20,200 & 32,700 & 56,600 & 82,200 & 116,000 & 244,000 \\
\hline 79 & 1.67 & 0364530 & 0961230 & E & $\mathrm{N}$ & 320 & 607 & 822 & 1,110 & 1,330 & 1,550 & 2,070 \\
\hline 80 & 139 & 0364310 & 0960756 & E & $\mathrm{N}$ & 8,260 & 13,300 & 16,600 & 20,400 & 23,100 & 25,600 & 30,900 \\
\hline 81 & 1,753 & 0363826 & 0955602 & E & $\mathrm{R}$ & 14,100 & 22,500 & 27,700 & 33,800 & 37,900 & 41,600 & 49,400 \\
\hline 82 & 0.94 & 0364340 & 0955052 & E & $\mathrm{N}$ & 353 & 517 & 618 & 737 & 818 & 895 & 1,060 \\
\hline 83 & 2.39 & 0363050 & 0955625 & E & $\mathrm{R}$ & 1,020 & 2,500 & 3,580 & 4,870 & 5,730 & 6,490 & 7,890 \\
\hline 84 & 1,955 & 0363032 & 0955030 & E & $\mathrm{R}$ & 19,100 & 34,200 & 48,000 & 70,800 & 92,500 & 119,000 & 204,000 \\
\hline \multirow[t]{2}{*}{85} & 6,534 & 0361825 & 0954152 & E & $\mathrm{N}$ & 43,900 & 73,900 & 96,400 & 127,000 & 152,000 & 178,000 & 243,000 \\
\hline & & & & & $\mathrm{R}$ & 24,300 & 34,500 & 41,000 & 49,000 & 54,900 & 60,600 & 73,600 \\
\hline 86 & 66.0 & 0363200 & 0960943 & E & $\mathrm{R}$ & 846 & 1,460 & 1,850 & 2,330 & 2,660 & 2,960 & 3,590 \\
\hline \multirow[t]{2}{*}{87} & 364 & 0362912 & 0960350 & E & $\mathrm{N}$ & 12,500 & 19,300 & 23,900 & 29,700 & 34,000 & 38,200 & 47,900 \\
\hline & & & & & $\mathrm{R}$ & 16,400 & 23,000 & 27,200 & 32,300 & 35,900 & 39,500 & 47,400 \\
\hline 88 & 30.6 & 0363206 & 0960254 & E & $\mathrm{N}$ & 5,190 & 7,910 & 9,700 & 11,900 & 13,500 & 15,100 & 18,600 \\
\hline 89 & 340 & 0362055 & 0960635 & E & $\mathrm{N}$ & 8,300 & 12,800 & 16,500 & 21,900 & 26,600 & 31,900 & 46,900 \\
\hline \multirow[t]{2}{*}{90} & 905 & 0361642 & 0955714 & E & $\mathrm{N}$ & 14,200 & 25,600 & 35,900 & 52,900 & 69,000 & 88,600 & 152,000 \\
\hline & & & & & $\mathrm{R}$ & 16,900 & 24,000 & 28,800 & 35,100 & 39,900 & 44,700 & 56,500 \\
\hline 91 & 8.20 & 0361255 & 0955942 & E & $\mathrm{U}$ & 1,910 & 3,050 & 3,870 & 4,980 & 5,830 & 6,700 & 8,860 \\
\hline 92 & 7.53 & 0361140 & 0955450 & E & $\mathrm{U}$ & 1,970 & 3,320 & 4,500 & 6,420 & 8,190 & 10,300 & 16,900 \\
\hline 93 & 1,022 & 0361455 & 0955206 & E & $\mathrm{R}$ & 16,300 & 21,700 & 25,200 & 29,700 & 33,000 & 36,200 & 44,000 \\
\hline 94 & 59.9 & 0361314 & 0955130 & E & $\mathrm{U}$ & 5,770 & 8,370 & 11,400 & 17,600 & 24,500 & 34,400 & 76,600 \\
\hline 95 & 1,103 & 0361323 & 0954909 & E & $\mathrm{R}$ & 17,400 & 22,000 & 24,600 & 27,400 & 29,200 & 30,900 & 34,300 \\
\hline 96 & 7,911 & 0360951 & 0953711 & E & $\mathrm{N}$ & 50,800 & 89,300 & 120,000 & 163,000 & 198,000 & 237,000 & 338,000 \\
\hline 97 & 11.1 & 0360850 & 0952705 & E & $\mathrm{N}$ & 901 & 1,410 & 1,780 & 2,280 & 2,670 & 3,090 & 4,140 \\
\hline 98 & 211 & 0371130 & 0951130 & E & $\mathrm{N}$ & 8,310 & 12,900 & 16,100 & 20,100 & 23,100 & 26,000 & 32,900 \\
\hline
\end{tabular}




\begin{tabular}{|c|c|c|c|c|c|c|c|}
\hline \multirow[b]{2}{*}{$\begin{array}{c}\text { Site } \\
\text { number } \\
\text { (fig. 1) }\end{array}$} & \multirow[b]{2}{*}{$\begin{array}{l}\text { Station } \\
\text { number }\end{array}$} & \multirow[b]{2}{*}{ Station name } & \multirow[b]{2}{*}{$\begin{array}{l}\text { Type of } \\
\text { station } \\
\text { (CNNT/ } \\
\text { CSG) }\end{array}$} & \multicolumn{3}{|c|}{ Documented extreme peak discharge } & \multirow[b]{2}{*}{$\begin{array}{c}\text { Potential } \\
\text { extreme } \\
\text { peak } \\
\text { discharge } \\
\text { (ttit/s) } \\
\text { (table 4) }\end{array}$} \\
\hline & & & & $\begin{array}{l}\text { Type of } \\
\text { record (H/ } \\
\text { I/N/ } \\
\text { R/U) }\end{array}$ & Date & $\begin{array}{c}\text { Discharge } \\
\left(\mathrm{ft}^{3} / \mathbf{s}\right)\end{array}$ & \\
\hline 98 & & & & $\mathrm{~N}$ & $06 / 22 / 48$ & $30,000^{\mathrm{a}}$ & \\
\hline 99 & 07184600 & Fly Creek near Faulkner, Kans. & CSG & $\mathrm{N}$ & 07/03/76 & 28,000 & 62,200 \\
\hline 100 & 07185000 & Neosho River near Commerce, Okla. & CONT & $\mathrm{N}$ & 07/15/51 & $267,000^{a, b}$ & 377,000 \\
\hline 101 & 07185095 & Tar Creek at 22nd Street Bridge, Miami, Okla. & CONT & $\mathrm{U}$ & 09/25/93 & $12,400^{\mathrm{c}}$ & 77,700 \\
\hline 102 & 07186000 & Spring River near Waco, Mo. ${ }^{4}$ & CONT & $\mathrm{N}$ & 09/26/93 & 151,000 & 231,000 \\
\hline 103 & 07186400 & Center Creek near Cartersville, Mo. & CONT & $\mathrm{N}$ & 07/03/76 & 36,300 & 142,000 \\
\hline 104 & 07187000 & Shoal Creek above Joplin, Mo. & CONT & $\mathrm{N}$ & $05 / 18 / 43$ & $62,100^{\mathrm{a}}$ & 171,000 \\
\hline 105 & 07188000 & Spring River near Quapaw, Okla. & CONT & $\mathrm{N}$ & 09/26/93 & $230,000^{\mathrm{c}}$ & 290,000 \\
\hline 106 & 07188140 & Flint Branch near Peoria, Okla. & CSG & $\mathrm{N}$ & $06 / 13 / 64$ & 4,400 & 25,400 \\
\hline 107 & 07188500 & Lost Creek at Seneca, Mo. ${ }^{5}$ & CSG & $\mathrm{N}$ & 10/02/59 & 20,000 & 75,700 \\
\hline 108 & 07188900 & Butler Creek Tributary near Gravette, Ark. & CSG & $\mathrm{N}$ & $05 / 19 / 61$ & 562 & 6,060 \\
\hline 109 & 07189000 & Elk River near Tiff City, Mo. & CONT & $\mathrm{N}$ & $04 / 19 / 41$ & $137,000^{a, b}$ & 213,000 \\
\hline \multirow[t]{2}{*}{110} & 07189480 & Wolf Creek near Grove, Okla. & CSG & $\mathrm{HN}$ & 05/00/43 & 7,500 & 32,600 \\
\hline & & & & $\mathrm{N}$ & $02 / 01 / 68$ & 1,500 & \\
\hline 111 & 07189500 & Neosho River near Grove, Okla. & CONT & $\mathrm{N}$ & $04 / 15 / 27$ & $133,000^{\mathrm{a}}$ & 440,000 \\
\hline 112 & 07189700 & Horse Creek at Afton, Okla. & CSG & $\mathrm{N}$ & 07/03/76 & 2,690 & 56,800 \\
\hline \multirow[t]{2}{*}{113} & 07190500 & Neosho River near Langley, Okla. & CONT & $\mathrm{HN}$ & 06/00/35 & 150,000 & 443,000 \\
\hline & & & & $\mathrm{R}$ & 05/20/43 & 300,000 & \\
\hline 114 & 07190600 & Big Cabin Creek near Pyramid Corners, Okla. ${ }^{6}$ & CSG & $\mathrm{N}$ & $03 / 108 / 74$ & 18,800 & 94,100 \\
\hline \multirow[t]{2}{*}{115} & 07191000 & Big Cabin Creek near Big Cabin, Okla. & CONT & $\mathrm{HN}$ & $05 / 18 / 43$ & $63,000^{\mathrm{a}}$ & 174,000 \\
\hline & & & & $\mathrm{N}$ & $10 / 03 / 59$ & 52,000 & \\
\hline 116 & 07191220 & Spavinaw Creek near Sycamore, Okla. & CONT & $\mathrm{N}$ & 07/27/75 & 39,800 & 118,000 \\
\hline 117 & 07191260 & Brushy Creek near Jay, Okla. & CSG & $\mathrm{N}$ & $11 / 24 / 73$ & 4,640 & 49,000 \\
\hline \multirow[t]{3}{*}{118} & 07191500 & Neosho River near Chouteau, Okla. & CONT & $\mathrm{HN}$ & $04 / 19 / 27$ & 165,000 & 456,000 \\
\hline & & & & $\mathrm{R}$ & 05/20/43 & 400,000 & \\
\hline & & & & $\mathrm{R}$ & $06 / 11 / 95$ & 164,000 & \\
\hline \multirow[t]{2}{*}{119} & 07192000 & Pryor Creek near Pryor, Okla. & CONT & $\mathrm{HN}$ & $05 / 10 / 43$ & 19,000 & 141,000 \\
\hline & & & & $\mathrm{N}$ & $10 / 03 / 59$ & 32,000 & \\
\hline \multirow[t]{2}{*}{120} & 07192500 & Neosho River near Wagoner, Okla. & CONT & $\mathrm{HN}$ & 04/16/27 & 170,000 & 464,000 \\
\hline & & & & $\mathrm{R}$ & $05 / 21 / 43$ & $400,000^{\mathrm{a}}$ & \\
\hline \multirow[t]{2}{*}{121} & 07193500 & Neosho River below Fort Gibson Lake & CONT & HR & $05 / 21 / 43$ & 400,000 & 465,000 \\
\hline & & near Fort Gibson, Okla. & & $\mathrm{R}$ & $05 / 26 / 57$ & 223,000 & \\
\hline \multirow[t]{3}{*}{122} & 07194500 & Arkansas River near Muskogee, Okla. & CONT & $\mathrm{HN}$ & 05/00/1898 & 384,000 & 770,000 \\
\hline & & & & $\mathrm{N}$ & $05 / 21 / 43$ & 700,000 & \\
\hline & & & & $\mathrm{R}$ & $05 / 26 / 57$ & 366,000 & \\
\hline 123 & 07194515 & Mill Creek near Park Hill, Okla. & CSG & $\mathrm{N}$ & $04 / 19 / 68$ & 1,860 & 15,100 \\
\hline 124 & 07195000 & Osage Creek near Elm Springs, Ark. & CONT & $\mathrm{N}$ & 05/10/50 & $22,500^{\mathrm{a}}$ & 117,000 \\
\hline 125 & 07195200 & Brush Creek Tributary near Tonitown, Ark. & CSG & $\mathrm{N}$ & 07/23/59 & 278 & 2,310 \\
\hline 126 & 07195450 & Ballard Creek at Summers, Ark. & CSG & $\mathrm{N}$ & $11 / 19 / 85$ & 5,100 & 47,200 \\
\hline 127 & 07195500 & Illinois River near Watts, Okla. & CONT & $\mathrm{N}$ & $07 / 25 / 60$ & 68,000 & 194,000 \\
\hline 128 & 07195800 & Flint Creek at Springtown, Ark. & CONT & $\mathrm{N}$ & 06/08/74 & 14,600 & 46,600 \\
\hline 129 & 07195855 & Flint Creek near West Siloam Springs, Okla. & CONT & $\mathrm{R}$ & 06/30/99 & 6,860 & 87,900 \\
\hline 130 & 07196000 & Flint Creek near Kansas, Okla. & CONT & $\mathrm{N}$ & $06 / 108 / 74$ & 44,400 & 110,000 \\
\hline
\end{tabular}

\begin{tabular}{|c|c|c|c|c|c|c|c|c|c|c|c|c|}
\hline \multirow{2}{*}{$\begin{array}{c}\text { Site } \\
\text { number } \\
\text { (fig. 1) }\end{array}$} & \multirow{2}{*}{$\begin{array}{c}\text { Contrib- } \\
\text { uting } \\
\text { drainage } \\
\text { area }\left(\mathrm{mi}^{2}\right)\end{array}$} & \multirow{2}{*}{ Latitude } & \multirow{2}{*}{ Longitude } & \multirow{2}{*}{$\begin{array}{l}\text { Hydrologic } \\
\text { region (E/ } \\
\text { W) }\end{array}$} & \multirow{2}{*}{$\begin{array}{c}\text { Type } \\
\text { basin } \\
\text { (N///R/ } \\
\text { U) }\end{array}$} & \multicolumn{7}{|c|}{$\begin{array}{l}\text { LPIII flood frequency estimates } \\
\text { Peak discharge for indicated recurrence interval }\left(\mathrm{ft}^{3} / \mathrm{s}\right)\end{array}$} \\
\hline & & & & & & $2 \mathrm{yr}$ & $5 \mathrm{yr}$ & $10 \mathrm{yr}$ & $25 \mathrm{yr}$ & $50 \mathrm{yr}$ & $100 \mathrm{yr}$ & $500 \mathrm{yr}$ \\
\hline 99 & 27.0 & 0370615 & 0945621 & $\mathrm{E}$ & $\mathrm{N}$ & 4,170 & 11,000 & 18,000 & 30,000 & 41,500 & 55,300 & 97,500 \\
\hline 100 & 5,876 & 0365543 & 0945726 & E & $\mathrm{N}$ & 34,200 & 59,800 & 82,200 & 118,000 & 150,000 & 188,000 & 302,000 \\
\hline & & & & & $\mathrm{R}$ & 37,900 & 57,000 & 71,200 & 90,700 & 106,000 & 123,000 & 167,000 \\
\hline 101 & 44.7 & 0365400 & 0945205 & E & $\mathrm{U}$ & 3,090 & 5,860 & 8,400 & 12,600 & 16,500 & 21,300 & 36,200 \\
\hline 102 & 1,164 & 0371444 & 0943358 & E & $\mathrm{N}$ & 18,700 & 34,400 & 47,100 & 65,800 & 81,600 & 98,900 & 146,000 \\
\hline 103 & 232 & 0370826 & 0942257 & E & $\mathrm{N}$ & 5,620 & 11,500 & 16,700 & 24,900 & 32,200 & 40,500 & 64,500 \\
\hline 104 & 427 & 0370123 & 0943558 & E & $\mathrm{N}$ & 7,350 & 15,000 & 21,700 & 32,000 & 41,000 & 51,200 & 79,700 \\
\hline 105 & 2,510 & 0365604 & 0944449 & E & $\mathrm{N}$ & 35,500 & 64,000 & 86,900 & 120,000 & 148,000 & 178,000 & 258,000 \\
\hline 106 & 4.90 & 0365225 & 0944135 & E & $\mathrm{N}$ & 786 & 1,480 & 2,060 & 2,940 & 3,690 & 4,530 & 6,860 \\
\hline 107 & 42.0 & 0365028 & 0943630 & E & $\mathrm{N}$ & 890 & 3,140 & 6,010 & 11,900 & 18,500 & 27,500 & 60,400 \\
\hline 108 & 0.96 & 0362651 & 0942636 & E & $\mathrm{N}$ & 101 & 296 & 495 & 828 & 1,130 & 1,490 & 2,480 \\
\hline 109 & 872 & 0363753 & 0943512 & E & $\mathrm{N}$ & 20,400 & 40,500 & 56,500 & 79,200 & 97,600 & 117,000 & 166,000 \\
\hline 110 & 7.21 & 0363720 & 0944450 & E & $\mathrm{N}$ & 1,130 & 1,620 & 1,960 & 2,410 & 2,750 & 3,090 & 3,940 \\
\hline 111 & 9,969 & 0363645 & 0944925 & E & $\mathrm{N}$ & 62,200 & 100,000 & 126,000 & 157,000 & 180,000 & 202,000 & 252,000 \\
\hline 112 & 21.9 & 0364150 & 0945720 & E & $\mathrm{N}$ & 1,680 & 2,120 & 2,380 & 2,700 & 2,920 & 3,130 & 3,610 \\
\hline 113 & 10,335 & 0362620 & 0950254 & E & $\mathrm{R}$ & 53,800 & 108,000 & 152,000 & 213,000 & 262,000 & 314,000 & 443,000 \\
\hline 114 & 71.1 & 0364806 & 0950948 & E & $\mathrm{N}$ & 4,710 & 8,470 & 11,400 & 15,700 & 19,200 & 23,000 & 32,900 \\
\hline 115 & 450 & 0363406 & 0950907 & E & $\mathrm{N}$ & 16,600 & 29,000 & 38,500 & 51,800 & 62,500 & 73,900 & 103,000 \\
\hline 116 & 133 & 0362007 & 0943827 & E & $\mathrm{N}$ & 3,480 & 8,730 & 13,600 & 21,000 & 27,500 & 34,600 & 53,500 \\
\hline 117 & 15.8 & 0362515 & 0944610 & E & $\mathrm{N}$ & 843 & 2,160 & 3,530 & 5,910 & 8,230 & 11,100 & 20,100 \\
\hline 118 & 11,534 & 0361346 & 0951057 & E & $\mathrm{R}$ & 56,400 & 90,200 & 117,000 & 156,000 & 189,000 & 226,000 & 327,000 \\
\hline 119 & 229 & 0361652 & 0951932 & E & $\mathrm{N}$ & 5,180 & 11,600 & 18,100 & 29,100 & 40,000 & 53,300 & 96,700 \\
\hline 120 & 12,307 & 0355544 & 0951608 & E & $\mathrm{R}$ & 115,000 & 221,000 & 285,000 & 352,000 & 392,000 & 424,000 & 479,000 \\
\hline 121 & 12,495 & 0355110 & 0951344 & E & $\mathrm{R}$ & 47,400 & 82,100 & 108,000 & 145,000 & 174,000 & 205,000 & 284,000 \\
\hline 122 & 84,133 & 0354610 & 0951749 & E & $\mathrm{N}$ & 161,000 & 236,000 & 284,000 & 342,000 & 383,000 & 422,000 & 509,000 \\
\hline 123 & 2.57 & 0354837 & 0950407 & E & $\mathrm{N}$ & 433 & 864 & 1,230 & 1,790 & 2,270 & 2,810 & 4,300 \\
\hline 124 & 130 & 0361319 & 0941718 & $\mathrm{E}$ & $\mathrm{N}$ & 4,980 & 10,200 & 14,800 & 21,800 & 27,900 & 34,700 & 53,800 \\
\hline 125 & 0.37 & 0361038 & 0941640 & E & $\mathrm{N}$ & 63 & 164 & 268 & 449 & 624 & 837 & 1,500 \\
\hline 126 & 14.6 & 0355842 & 0942956 & E & $\mathrm{N}$ & 1,700 & 4,010 & 6,040 & 9,090 & 11,600 & 14,400 & 21,600 \\
\hline 127 & 635 & 0360748 & 0943419 & E & $\mathrm{N}$ & 18,900 & 33,300 & 43,400 & 56,200 & 65,700 & 75,000 & 96,000 \\
\hline 128 & 14.2 & 0361520 & 0942550 & E & $\mathrm{N}$ & 786 & 2,090 & 3,430 & 5,780 & 8,060 & 10,800 & 19,500 \\
\hline 129 & 59.8 & 0361258 & 0943615 & E & $\mathrm{R}$ & 1,270 & 3,300 & 5,260 & 8,450 & 11,300 & 14,600 & 23,800 \\
\hline 130 & 110 & 0361111 & 0944224 & E & $\mathrm{N}$ & 3.950 & 9,750 & 15,200 & 23,700 & 31,300 & 39.800 & 63,300 \\
\hline
\end{tabular}




\begin{tabular}{|c|c|c|c|c|c|c|c|}
\hline \multirow[b]{2}{*}{$\begin{array}{c}\text { Site } \\
\text { number } \\
\text { (fig. 1) }\end{array}$} & \multirow[b]{2}{*}{$\begin{array}{l}\text { Station } \\
\text { number }\end{array}$} & \multirow[b]{2}{*}{ Station name } & \multirow[b]{2}{*}{$\begin{array}{l}\text { Type of } \\
\text { station } \\
\text { (CONT/ } \\
\text { CSG) }\end{array}$} & \multicolumn{3}{|c|}{ Documented extreme peak discharge } & \multirow[b]{2}{*}{$\begin{array}{c}\text { Potentia } \\
\text { extreme } \\
\text { peak } \\
\text { discharg } \\
\text { (ft/3/s) } \\
\text { (table 4) }\end{array}$} \\
\hline & & & & 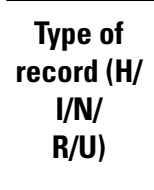 & Date & $\begin{array}{c}\text { Discharge } \\
\left(\mathbf{f t}^{3} / \mathbf{s}\right)\end{array}$ & \\
\hline 131 & 07196380 & Steely Hollow near Tahlequah, Okla. & CSG & $\mathrm{N}$ & $06 / 08 / 74$ & 5,000 & 20,000 \\
\hline 132 & 07196500 & Illinois River near Tahlequah, Okla. & CONT & $\begin{array}{c}\mathrm{HN} \\
\mathrm{N}\end{array}$ & $\begin{array}{l}01 / 00 / 16 \\
05 / 10 / 50\end{array}$ & $\begin{array}{l}112,000 \\
150,000 \text { a,b }\end{array}$ & 219,000 \\
\hline 133 & 07196900 & Baron Fork at Dutch Mills, Ark. & CONT & $\mathrm{N}$ & $11 / 18 / 85$ & 20,900 & 74,600 \\
\hline 134 & 07197000 & Baron Fork at Eldon, Okla. & CONT & $\mathrm{N}$ & $05 / 03 / 90$ & 50,600 & 155,000 \\
\hline 135 & 07198000 & Illinois River near Gore, Okla. ${ }^{7}$ & CONT & $\begin{array}{l}\mathrm{N} \\
\mathrm{R}\end{array}$ & $\begin{array}{l}05 / 11 / 50 \\
06 / 109 / 57\end{array}$ & $\begin{array}{c}180,000^{\mathrm{a}} \\
18,100\end{array}$ & 256,000 \\
\hline 136 & 07198500 & Dirty Creek near Warner, Okla. & CONT & $\begin{array}{c}\mathrm{HN} \\
\mathrm{N}\end{array}$ & $\begin{array}{l}02 / 00 / 38 \\
05 / 10 / 43\end{array}$ & $\begin{array}{l}19,300 \\
42,000^{a}\end{array}$ & 141,000 \\
\hline 137 & 07228290 & Rough Creek near Thomas, Okla. & CSG & $\mathrm{N}$ & 05/23/81 & 6,270 & 26,200 \\
\hline 138 & 07228450 & Deer Creek Tributary near Hydro, Okla. & CSG & $\mathrm{N}$ & $09 / 21 / 65$ & 1,050 & 8,230 \\
\hline 139 & 07228500 & Canadian River at Bridgeport, Okla. & CONT & $\begin{array}{l}\mathrm{N} \\
\mathrm{R}\end{array}$ & $\begin{array}{l}06 / 23 / 48 \\
05 / 17 / 82\end{array}$ & $\begin{array}{r}150,000 \\
86,100\end{array}$ & 287,000 \\
\hline 140 & 07228930 & Worley Creek near Tuttle, Okla. & CSG & $\mathrm{N}$ & 10/20/83 & 3,000 & 41,500 \\
\hline 141 & 07228960 & Canadian River Tributary near Newcastle, Okla. & CSG & $\mathrm{N}$ & $04 / 14 / 65$ & 1,460 & 18,800 \\
\hline 142 & 07229100 & Canadian River near Noble, Okla. & CONT & $\mathrm{R}$ & $09 / 22 / 65$ & 35,500 & 536,000 \\
\hline 143 & 07229200 & Canadian River at Purcell, Okla. & CONT & $\mathrm{R}$ & 05/29/87 & 102,000 & 536,000 \\
\hline 144 & 07229220 & Walnut Creek near Blanchard, Okla. & CSG & $\mathrm{N}$ & $11 / 20 / 63$ & 887 & 7,820 \\
\hline 145 & 07229300 & Walnut Creek near Purcell, Okla. & CONT & $\mathrm{N}$ & 10/20/83 & $67,700 \mathrm{~g}$ & 136,000 \\
\hline 146 & 07229420 & Julian Creek Tributary near Asher, Okla. & CSG & $\mathrm{N}$ & $05 / 13 / 68$ & 2,000 & 13,600 \\
\hline 147 & 07229430 & Arbeca Creek near Allen, Okla. & CSG & $\mathrm{N}$ & $10 / 08 / 70$ & 2,600 & 13,500 \\
\hline 148 & 07230000 & Little River blw Lk Thunderbird near Norman, Okla. ${ }^{8}$ & CONT & $\begin{array}{l}\mathrm{N} \\
\mathrm{R}\end{array}$ & $\begin{array}{l}05 / 25 / 57 \\
051\end{array}$ & $\begin{array}{c}34,600^{\mathrm{a}} \\
1.40\end{array}$ & 146,000 \\
\hline 149 & 07230500 & Little River near Tecumseh, Okla. & CONT & $\begin{array}{c}\mathrm{R} \\
\mathrm{HN} \\
\mathrm{N}\end{array}$ & $\begin{array}{l}05 / 10 / 90 \\
06 / / 00 / 32 \\
05 / 25 / 57\end{array}$ & $\begin{array}{l}1,450 \\
60,000^{\mathrm{a}} \\
623,400\end{array}$ & 175,000 \\
\hline & & & & $\begin{array}{l}\mathrm{N} \\
\mathrm{R}\end{array}$ & $0505 / 153 / 97$ & $14,000^{\mathrm{h}}$ & \\
\hline 150 & 07231000 & Little River near Sasakwa, Okla. & CONT & HN & $06 / 00 / 39$ & 33,000 & 213,000 \\
\hline & & & & $\mathrm{N}$ & $05 / 11 / 50$ & $44,600^{\mathrm{a}}$ & \\
\hline & & & & $\mathrm{R}$ & $05 / 01 / 85$ & 18,500 & \\
\hline 151 & 07231320 & Leader Creek Tributary near Atwood, Okla. & CSG & $\mathrm{N}$ & $10 / 08 / 70$ & 1,470 & 4,610 \\
\hline 152 & 07231500 & Canadian River at Calvin, Okla. & CONT & HN & $08 / 07 / 06$ & 128,000 & 548,000 \\
\hline & & & & $\mathrm{HN}$ & $05 / 31 / 37$ & 135,000 & \\
\hline & & & & $\mathrm{N}$ & $05 / 11 / 50$ & 174,000 & \\
\hline & & & & $\mathrm{R}$ & 05/29/87 & 154,000 & \\
\hline 153 & 07231560 & Middle Creek near Carson, Okla. & CSG & $\mathrm{N}$ & $05 / 13 / 68$ & 5,460 & 33,100 \\
\hline 154 & 07231950 & Pine Creek near Higgins, Okla. & CSG & $\mathrm{N}$ & 03/27/77 & 18,000 & 39,500 \\
\hline 155 & 07232000 & Gaines Creek near Krebs, Okla. & CONT & $\mathrm{HN}$ & $02 / 18 / 38$ & $70,000^{\mathrm{a}}$ & 190,000 \\
\hline & & & & $\mathrm{N}$ & $05 / 11 / 43$ & 62,000 & \\
\hline 156 & 07232500 & Beaver River near Guymon, Okla. ${ }^{9}$ & CONT & $\mathrm{HN}$ & 06/00/37 & 28,600 & 192,000 \\
\hline & & & & $\mathrm{N}$ & $06 / 15 / 64$ & 55,400 & \\
\hline & & & & I & $09 / 15 / 88$ & 3,410 & \\
\hline 157 & 07232650 & Aqua Frio Creek near Felt, Okla. & CSG & $\mathrm{N}$ & $08 / 19 / 65$ & 1,900 & 52,800 \\
\hline 158 & 07232900 & Coldwater Creek near Guymon, Okla. ${ }^{9}$ & CONT & I & $06 / 20 / 82$ & 5,800 & 174,000 \\
\hline 159 & 07233000 & Coldwater Creek near Hardesty, Okla. ${ }^{9}$ & CONT & $\mathrm{N}$ & $06 / 25 / 47$ & 21,500 & 176,000 \\
\hline 160 & 07233500 & Palo Duro Creek Near Spearman, Tex. & CONT & HN & $09 / 04 / 38$ & $34,000^{\mathrm{a}}$ & 185,000 \\
\hline & & & & $\mathrm{N}$ & 10/07/46 & 21,200 & \\
\hline
\end{tabular}

\begin{tabular}{|c|c|c|c|c|c|c|c|c|c|c|c|c|}
\hline \multirow{2}{*}{$\begin{array}{l}\text { Site } \\
\text { number } \\
\text { (fig. 1) }\end{array}$} & \multirow{2}{*}{$\begin{array}{c}\text { Contrib- } \\
\text { uting } \\
\text { drainage } \\
\text { area }\left(\mathrm{mi}^{2}\right)\end{array}$} & \multirow{2}{*}{ Latitude } & \multirow{2}{*}{ Longitude } & \multirow{2}{*}{$\begin{array}{l}\text { Hydrologic } \\
\text { region (E/ } \\
\text { W) }\end{array}$} & \multirow{2}{*}{$\begin{array}{c}\text { Type basin } \\
\text { (N///R/ } \\
\text { U) }\end{array}$} & \multicolumn{7}{|c|}{$\begin{array}{l}\text { LPIII flood frequency estimates } \\
\text { Peak discharge for indicated recurrence interval }\left(\mathrm{tt}^{3} / \mathrm{s}\right)\end{array}$} \\
\hline & & & & & & $2 \mathrm{yr}$ & $5 \mathrm{yr}$ & $10 \mathrm{yr}$ & $25 \mathrm{yr}$ & $50 \mathrm{yr}$ & $100 \mathrm{yr}$ & $500 \mathrm{yr}$ \\
\hline 131 & 3.59 & 0355837 & 0945524 & E & $\mathrm{N}$ & 536 & 1,760 & 3,190 & 5,880 & 8,630 & 12,100 & 23,300 \\
\hline 132 & 959 & 0355522 & 0945524 & E & $\mathrm{N}$ & 19,800 & 39,100 & 54,900 & 78,100 & 97,500 & 119,000 & 174,000 \\
\hline 133 & 40.6 & 0355248 & 0942911 & E & $\mathrm{N}$ & 7,110 & 14,000 & 19,400 & 26,900 & 32,700 & 38,800 & 53,400 \\
\hline 134 & 307 & 0355516 & 0945018 & E & $\mathrm{N}$ & 15,200 & 26,300 & 34,300 & 44,800 & 52,900 & 61,000 & 80,200 \\
\hline \multirow[t]{2}{*}{135} & 1,626 & 0353423 & 0950407 & E & $\mathrm{N}$ & 31,300 & 64,300 & 94,300 & 143,000 & 187,000 & 239,000 & 395,000 \\
\hline & & & & & $\mathrm{R}$ & 8,180 & 11,900 & 14,300 & 17,100 & 19,100 & 21,000 & 25,100 \\
\hline 136 & 227 & 0353318 & 0951828 & E & $\mathrm{N}$ & 9,170 & 23,700 & 39,200 & 67,400 & 96,000 & 132,000 & 254,000 \\
\hline 137 & 10.4 & 0354808 & 0984715 & w & $\mathrm{N}$ & 794 & 2,170 & 3,690 & 6,500 & 9,390 & 13,100 & 25,600 \\
\hline 138 & 2.31 & 0353210 & 0982850 & W & $\mathrm{N}$ & 304 & 539 & 741 & 1,060 & 1,340 & 1,670 & 2,640 \\
\hline \multirow[t]{2}{*}{139} & 20,475 & 0353237 & 0981903 & W & $\mathrm{N}$ & 26,200 & 45,300 & 60,000 & 80,900 & 97,900 & 116,000 & 164,000 \\
\hline & & & & & $\mathrm{R}$ & 16,200 & 31,400 & 43,800 & 61,700 & 76,600 & 92,700 & 135,000 \\
\hline 140 & 11.2 & 0351728 & 0974510 & E & $\mathrm{N}$ & 1,260 & 2,130 & 2,800 & 3,770 & 4,570 & 5,450 & 7,780 \\
\hline 141 & 3.32 & 0351727 & 0973720 & E & $\mathrm{N}$ & 710 & 1,180 & 1,550 & 2,090 & 2,550 & 3,060 & 4,470 \\
\hline 142 & 21,110 & 0350455 & 0972252 & E & $\mathrm{R}$ & 15,700 & 24,100 & 30,800 & 40,400 & 48,400 & 57,300 & 81,800 \\
\hline 143 & 21,138 & 0350050 & 0972050 & E & $\mathrm{R}$ & 21,400 & 43,200 & 62,700 & 93,700 & 122,000 & 154,000 & 250,000 \\
\hline 144 & 1.26 & 0350720 & 0974210 & E & $\mathrm{N}$ & 378 & 666 & 906 & 1,270 & 1,590 & 1,960 & 3,000 \\
\hline 145 & 202 & 0345956 & 0972200 & E & $\mathrm{N}$ & 8,750 & 16,900 & 24,500 & 37,400 & 49,700 & 64,800 & 114,000 \\
\hline 146 & 2.28 & 0345909 & 0965848 & E & $\mathrm{N}$ & 400 & 746 & 1,070 & 1,610 & 2,140 & 2,780 & 4,870 \\
\hline 147 & 2.26 & 0345410 & 0962320 & E & $\mathrm{N}$ & 660 & 1,210 & 1,690 & 2,490 & 3,220 & 4,100 & 6,830 \\
\hline \multirow[t]{2}{*}{148} & 257 & 0351318 & 0971249 & E & $\mathrm{N}$ & 5,300 & 8,500 & 11,200 & 15,500 & 19,400 & 23,900 & 37,700 \\
\hline & & & & & $\mathrm{R}$ & 667 & 960 & 1,120 & 1,300 & 1,410 & 1,510 & 1,690 \\
\hline \multirow[t]{2}{*}{149} & 456 & 0351021 & 0965554 & E & $\mathrm{N}$ & 9,200 & 16,800 & 24,000 & 36,500 & 48,800 & 64,100 & 116,000 \\
\hline & & & & & $\mathrm{R}$ & 5,040 & 7,230 & 8,810 & 11,000 & 12,600 & 14,400 & 19,000 \\
\hline \multirow[t]{2}{*}{150} & 865 & 0345902 & 0963301 & E & $\mathrm{N}$ & 15,200 & 26,900 & 36,700 & 51,500 & 64,500 & 79,300 & 122,000 \\
\hline & & & & & $\mathrm{R}$ & 7,820 & 11,900 & 14,600 & 17,700 & 19,900 & 22,000 & 26,600 \\
\hline 151 & 0.72 & 0345710 & 0962040 & E & $\mathrm{N}$ & 300 & 590 & 868 & 1,330 & 1,780 & 2,330 & 4,110 \\
\hline \multirow{2}{*}{152} & 23,151 & 0345840 & 0961436 & E & $\mathrm{N}$ & 60,400 & 94,700 & 121,000 & 157,000 & 186,000 & 218,000 & 300,000 \\
\hline & & & & & $\mathrm{R}$ & 53,300 & 88,900 & 114,000 & 147,000 & 172,000 & 198,000 & 258,000 \\
\hline 153 & 7.40 & 0351110 & 0960420 & E & $\mathrm{N}$ & 1,650 & 3,030 & 4,260 & 6,200 & 7,980 & 10,100 & 16,400 \\
\hline 154 & 9.99 & 0344740 & 0952050 & E & $\mathrm{N}$ & 4,150 & 7,940 & 11,200 & 16,300 & 20,800 & 26,000 & 40,900 \\
\hline 155 & 588 & 0345900 & 0953700 & E & $\mathrm{N}$ & 11,600 & 20,500 & 28,300 & 40,900 & 52,500 & 66,300 & 108,000 \\
\hline \multirow[t]{2}{*}{156} & 1,175 & 0364317 & 1012921 & W & $\mathrm{N}$ & 8,580 & 21,400 & 33,100 & 51,000 & 66,300 & 82,900 & 126,000 \\
\hline & & & & & I & 181 & 1,160 & 2,510 & 5,020 & 7,340 & 9,910 & 16,300 \\
\hline 157 & 31.0 & 0363323 & 10247 & $\mathrm{~W}$ & $\mathrm{~N}$ & 131 & 706 & 1,690 & 4,270 & 7,750 & 13,200 & 38,600 \\
\hline 158 & 725 & 0363419 & 1012252 & w & I & 223 & 979 & 1,980 & 4,010 & 6,150 & 8,890 & 17,900 \\
\hline 159 & 767 & 0363838 & 1011238 & W & $\mathrm{N}$ & 2,720 & 7,460 & 12,300 & 20,500 & 28,200 & 37,200 & 64,100 \\
\hline 160 & 960 & 0361208 & 1011820 & $\mathrm{w}$ & $\mathrm{N}$ & 2,400 & 6,510 & 11,200 & 20,100 & 29,500 & 42,000 & 86,700 \\
\hline
\end{tabular}




\begin{tabular}{|c|c|c|c|c|c|c|c|}
\hline \multirow[b]{2}{*}{$\begin{array}{c}\text { Site } \\
\text { number } \\
\text { (fig. 1) }\end{array}$} & \multirow[b]{2}{*}{$\begin{array}{l}\text { Station } \\
\text { number }\end{array}$} & \multirow[b]{2}{*}{ Station name } & \multirow[b]{2}{*}{$\begin{array}{l}\text { Type of } \\
\text { station } \\
\text { (CNNT/ } \\
\text { CSG) }\end{array}$} & \multicolumn{3}{|c|}{ Documented extreme peak discharge } & \multirow[b]{2}{*}{$\begin{array}{c}\text { Potential } \\
\text { extreme } \\
\text { peak } \\
\text { discharge } \\
\left(\mathbf{f t}^{3} / \mathrm{s}\right) \\
\text { (table 4) }\end{array}$} \\
\hline & & & & $\begin{array}{c}\text { Type of } \\
\text { record (H/ } \\
\text { I/N/ } \\
\text { R/U) }\end{array}$ & Date & $\begin{array}{c}\text { Discharge } \\
\left(\mathrm{ft}^{3} / \mathbf{s}\right)\end{array}$ & \\
\hline 161 & 07233850 & Sharp Creek Tributary near Turpin, Okla. & CSG & $\mathrm{N}$ & $03 / 23 / 73$ & 120 & 4,020 \\
\hline \multirow{2}{*}{162} & 07234000 & Beaver River at Beaver, Okla. ${ }^{9}$ & CONT & $\mathrm{N}$ & $10 / 08 / 46$ & $70,000^{\mathrm{a}}$ & 232,000 \\
\hline & & & & IR & 06/10/83 & 5,510 & \\
\hline 163 & 07234050 & North Fork Clear Creek near Balko, Okla. & CSG & $\mathrm{N}$ & 08/22/65 & 1,800 & 13,400 \\
\hline 164 & 07234100 & Clear Creek near Elmwood, Okla. & CONT & $\mathrm{N}$ & $05 / 17 / 89$ & 20,700 & 112,000 \\
\hline 165 & 07234150 & White Woman Creek Tributary near Doarrouzett, Tex. & CONT & $\mathrm{N}$ & $08 / 31 / 66$ & 416 & 13,000 \\
\hline 166 & 07234290 & Clear Creek Tributary near Catesby, Okla. & CSG & $\mathrm{N}$ & 06/09/68 & 1,480 & 22,900 \\
\hline 167 & 07234500 & Beaver River near Fort Supply, Okla. & CONT & $\mathrm{N}$ & 10/09/46 & $50,000^{\mathrm{a}}$ & 242,000 \\
\hline \multirow[t]{3}{*}{168} & 07235000 & Wolf Creek at Lipscomb, Tex. ${ }^{9}$ & CONT & $\mathrm{N}$ & 10/21/41 & $20,000^{\mathrm{a}}$ & 156,000 \\
\hline & & & & $\mathrm{R}$ & $05 / 31 / 63$ & 8,790 & \\
\hline & & & & IR & 09/19/96 & 10,300 & \\
\hline 169 & 07235700 & Table 2. Little Wolf Creek near Gage, Okla. & CSG & $\mathrm{N}$ & $05 / 13 / 69$ & 5,200 & 37,800 \\
\hline 170 & 07236000 & Wolf Creek near Fargo, Okla. ${ }^{9}$ & CONT & $\mathrm{N}$ & 06/23/57 & $81,600^{\mathrm{a}}$ & 197,000 \\
\hline \multirow[t]{3}{*}{171} & 07237000 & Wolf Creek near Fort Supply, Okla. ${ }^{9}$ & CONT & $\mathrm{N}$ & $06 / 24 / 39$ & 14,200 & 200,000 \\
\hline & & & & $\mathrm{R}$ & 05/19/57 & 5,020 & \\
\hline & & & & IR & $05 / 13 / 79$ & 2,010 & \\
\hline \multirow[t]{2}{*}{172} & 07237500 & North Canadian River at Woodward, Okla. ${ }^{9}$ & CONT & $\mathrm{N}$ & $10 / 10 / 46$ & 42,000 & 252,000 \\
\hline & & & & IR & 05/23/89 & 3,090 & \\
\hline 173 & 07237750 & Cottonwood Creek near Vici, Okla. & CSG & $\mathrm{N}$ & $05 / 13 / 75$ & 2,050 & 28,000 \\
\hline 174 & 07237800 & Bent Creek near Seiling, Okla. ${ }^{1}$ & CSG & $\mathrm{N}$ & 08/27/74 & 9,120 & 103,000 \\
\hline \multirow[t]{2}{*}{175} & 07238000 & North Canadian River near Seiling, Okla. ${ }^{9}$ & CONT & $\mathrm{N}$ & $05 / 19 / 51$ & 33,000 & 255,000 \\
\hline & & & & IR & 09/23/97 & 7,200 & \\
\hline \multirow[t]{2}{*}{176} & 07239000 & North Canadian River at Canton, Okla. & CONT & $\mathrm{N}$ & $10 / 12 / 46$ & $24,800^{\mathrm{a}}$ & 255,000 \\
\hline & & & & $\mathrm{R}$ & $06 / 11 / 49$ & 4,020 & \\
\hline 177 & 07239050 & North Canadian River Tributary near Eagle City, Okla. & CSG & $\mathrm{N}$ & $06 / 11 / 67$ & 501 & 2,180 \\
\hline 178 & 07239300 & North Canadian R. blw Weavers Ck near Watonga, Okla. & CONT & $\mathrm{R}$ & 10/03/86 & 9,740 & 256,000 \\
\hline 179 & 07239450 & North Canadian River near Calumet, Okla. & CONT & $\mathrm{R}$ & 05/10/93 & 9,310 & 412,000 \\
\hline \multirow[t]{2}{*}{180} & 07239500 & North Canadian River near El Reno, Okla. & CONT & $\mathrm{N}$ & $10 / 28 / 41$ & $15,000^{\mathrm{a}}$ & 413,000 \\
\hline & & & & $\mathrm{R}$ & 05/10/93 & $14,600^{\mathrm{c}}$ & \\
\hline \multirow[t]{2}{*}{181} & 07241000 & North Canadian River below Lake Overholser & CONT & HR & 10/00/23 & 135,000 & 416,000 \\
\hline & & near Oklahoma City, Okla. & & $\mathrm{R}$ & $06 / 11 / 95$ & 19,500 & \\
\hline \multirow[t]{2}{*}{182} & 07241500 & North Canadian River near Oklahoma City, Okla. ${ }^{10}$ & CONT & $\mathrm{HR}$ & 06/03/32 & 100,000 & 417,000 \\
\hline & & & & $\mathrm{R}$ & 10/30/41 & 16,700 & \\
\hline 183 & 07241520 & North Canadian River at Britton Rd at OKC, Okla. & CONT & $\mathrm{R}$ & 05/09/93 & $38,100^{\mathrm{c}}$ & 418,000 \\
\hline 184 & 07241550 & North Canadian River near Harrah, Okla. & CONT & $\mathrm{R}$ & $05 / 29 / 87$ & 27,200 & 419,000 \\
\hline 185 & 07241880 & Sand Creek near Cromwell, Okla. & CSG & $\mathrm{N}$ & 04/30/85 & 3,840 & 38,200 \\
\hline 186 & 07242000 & North Canadian River near Wetumka, Okla. & CONT & $\mathrm{R}$ & $04 / 15 / 45$ & $66,000^{\mathrm{a}}$ & 431,000 \\
\hline 187 & 07242160 & Alabama Creek near Weleetka, Okla. & CSG & $\mathrm{N}$ & $10 / 01 / 86$ & 4,910 & 50,000 \\
\hline 188 & 07242180 & Stidham Creek Tributary near Dustin, Okla. & CSG & $\mathrm{N}$ & $05 / 13 / 68$ & 622 & 15,000 \\
\hline \multirow[t]{2}{*}{189} & 07242350 & Deep Fork near Arcadia, Okla. & CONT & $\mathrm{U}$ & $11 / 02 / 74$ & 14,300 & 109,000 \\
\hline & & & & UR & $06 / 28 / 89$ & 1,470 & \\
\hline \multirow{2}{*}{190} & 07242380 & Deep Fork near Warwick, Okla. & CONT & $\mathrm{N}$ & 10/21/83 & 28,700 & 183,000 \\
\hline & & & & $\mathrm{R}$ & 06/09/95 & 34,600 & \\
\hline 191 & 07242500 & Bellcow Creek at Chandler, Okla. & CONT & $\mathrm{N}$ & $05 / 23 / 52$ & $2,910^{\mathrm{a}}$ & 78,800 \\
\hline
\end{tabular}

\begin{tabular}{|c|c|c|c|c|c|c|c|c|c|c|c|c|}
\hline \multirow{2}{*}{$\begin{array}{c}\text { Site } \\
\text { number } \\
\text { (fig. 1) }\end{array}$} & \multirow{2}{*}{$\begin{array}{c}\text { Contrib- } \\
\text { uting } \\
\text { drainage } \\
\text { area }\left(\mathrm{mi}^{2}\right)\end{array}$} & \multirow{2}{*}{ Latitude } & \multirow{2}{*}{ Longitude } & \multirow{2}{*}{$\begin{array}{l}\text { Hydro- } \\
\text { logic } \\
\text { region (E/ } \\
\text { W) }\end{array}$} & \multirow{2}{*}{$\begin{array}{c}\text { Type } \\
\text { basin (N/ } \\
\text { I/R/ } \\
\text { U) }\end{array}$} & \multicolumn{7}{|c|}{$\begin{array}{l}\text { LPIII flood frequency estimates } \\
\text { Peak discharge for indicated recurrence interval }\left(\mathrm{ft}^{3} / \mathrm{s}\right)\end{array}$} \\
\hline & & & & & & $2 \mathrm{yr}$ & $5 \mathrm{yr}$ & $10 \mathrm{yr}$ & $25 \mathrm{yr}$ & $50 \mathrm{yr}$ & $100 \mathrm{yr}$ & $500 \mathrm{yr}$ \\
\hline 161 & 1.00 & 0365150 & 1005445 & $\mathrm{~W}$ & $\mathrm{~N}$ & 34 & 71 & 101 & 145 & 181 & 221 & 323 \\
\hline \multirow{2}{*}{162} & 3,685 & 0364920 & 1003108 & w & $\mathrm{N}$ & 9,100 & 18,500 & 27,300 & 41,800 & 55,600 & 72,100 & 124,000 \\
\hline & & & & & IR & 838 & 2,310 & 3,600 & 5,450 & 6,890 & 8,350 & 11,700 \\
\hline 163 & 4.22 & 0363701 & 1003950 & w & $\mathrm{N}$ & 59 & 306 & 691 & 1,590 & 2,660 & 4,180 & 10,100 \\
\hline 164 & 170 & 0363852 & 1003007 & w & $\mathrm{N}$ & 1,290 & 4,710 & 9,040 & 17,700 & 27,100 & 39,400 & 82,400 \\
\hline 165 & 4.03 & 0362400 & 1001630 & w & $\mathrm{N}$ & 78 & 210 & 346 & 584 & 813 & 1,090 & 1,950 \\
\hline 166 & 8.57 & 0362930 & 0995720 & $\mathrm{w}$ & $\mathrm{N}$ & 113 & 462 & 920 & 1,860 & 2,870 & 4,210 & 8,800 \\
\hline 167 & 5,068 & 0363530 & 0993530 & w & $\mathrm{N}$ & 9,640 & 19,400 & 27,800 & 40,500 & 51,400 & 63,600 & 97,200 \\
\hline \multirow{2}{*}{168} & 475 & 0361416 & 1001630 & w & $\mathrm{R}$ & 3,240 & 7,040 & 10,100 & 14,400 & 17,900 & 21,400 & 30,000 \\
\hline & & & & & IR & 259 & 1,530 & 3,830 & 10,100 & 18,800 & 32,800 & 100,000 \\
\hline 169 & 17.8 & 0361426 & 0994630 & $\mathrm{w}$ & $\mathrm{N}$ & 495 & 1,400 & 2,380 & 4,170 & 5,980 & 8,250 & 15,700 \\
\hline 170 & 1,386 & 0362357 & 0993722 & $\mathrm{w}$ & $\mathrm{N}$ & 4,300 & 9,040 & 13,500 & 20,800 & 27,700 & 35,900 & 61,400 \\
\hline \multirow{2}{*}{171} & 1,498 & 0363400 & 0993305 & $\mathrm{w}$ & $\mathrm{R}$ & 924 & 2,170 & 3,170 & 4,550 & 5,600 & 6,650 & 9,020 \\
\hline & & & & & IR & 306 & 735 & 1,160 & 1,900 & 2,600 & 3,460 & 6,140 \\
\hline \multirow{2}{*}{172} & 6,777 & 0362612 & 0991641 & w & $\mathrm{N}$ & 5,330 & 12,400 & 19,400 & 31,300 & 42,600 & 56,200 & 98,700 \\
\hline & & & & & IR & 759 & 1,460 & 1,980 & 2,680 & 3,220 & 3,760 & 5,030 \\
\hline 173 & 11.5 & 0360845 & 0991200 & W & $\mathrm{N}$ & 436 & 1,010 & 1,540 & 2,380 & 3,130 & 3,980 & 6,380 \\
\hline 174 & 139 & 0361126 & 0990036 & w & $\mathrm{N}$ & 2,280 & 4,370 & 6,080 & 8,610 & 10,700 & 13,100 & 19,300 \\
\hline \multirow{2}{*}{175} & 7,414 & 0361100 & 0985515 & w & $\mathrm{N}$ & 4,250 & 8,800 & 13,200 & 20,800 & 28,200 & 37,400 & 67,600 \\
\hline & & & & & IR & 2,010 & 3,720 & 5,050 & 6,900 & 8,390 & 9,960 & 13,900 \\
\hline \multirow[t]{2}{*}{176} & 7,601 & 0360437 & 0983547 & w & $\mathrm{N}$ & 8,000 & 13,800 & 18,500 & 25,600 & 31,700 & 38,500 & 57,800 \\
\hline & & & & & $\mathrm{R}$ & 1,100 & 1,610 & 2,080 & 2,880 & 3,650 & 4,610 & 7,810 \\
\hline 177 & 0.52 & 0355530 & 0983500 & w & $\mathrm{N}$ & 89 & 228 & 378 & 649 & 924 & 1,270 & 2,460 \\
\hline 178 & 7,837 & 0354843 & 0982514 & w & $\mathrm{R}$ & 2,300 & 4,040 & 5,270 & 6,860 & 8,040 & 9,210 & 11,900 \\
\hline 179 & 8,063 & 0353701 & 0980354 & E & $\mathrm{R}$ & 3,060 & 5,270 & 7,090 & 9,830 & 12,200 & 14,900 & 22,400 \\
\hline \multirow{2}{*}{180} & 8,143 & 0353347 & 0975726 & E & $\mathrm{N}$ & 4,780 & 7,180 & 9,000 & 11,600 & 13,700 & 16,000 & 22,200 \\
\hline & & & & & $\mathrm{R}$ & 3,220 & 5,760 & 7,790 & 10,800 & 13,200 & 16,000 & 23,200 \\
\hline 181 & 8,323 & 0352843 & 0973947 & E & $\mathrm{R}$ & 3,490 & 9,110 & 14,500 & 23,200 & 30,900 & 39,600 & 63,900 \\
\hline 182 & 8,455 & 0352940 & 0972540 & E & $\mathrm{R}$ & 4,860 & 8,190 & 11,800 & 18,600 & 26,100 & 36,400 & 77,600 \\
\hline 183 & 8,514 & 0353356 & 0972201 & E & $\mathrm{R}$ & 12,700 & 24,200 & 33,200 & 45,900 & 56,100 & 66,900 & 93,900 \\
\hline 184 & 8,602 & 0353001 & 0971137 & E & $\mathrm{R}$ & 6,450 & 11,700 & 16,200 & 23,300 & 29,700 & 37,000 & 58,800 \\
\hline 185 & 9.48 & 0352056 & 0962940 & E & $\mathrm{N}$ & 1,400 & 2,160 & 2,760 & 3,610 & 4,340 & 5,130 & 7,310 \\
\hline 186 & 9,391 & 0351556 & 0961221 & E & $\mathrm{R}$ & 11,900 & 19,800 & 26,100 & 35,300 & 43,100 & 51,800 & 75,700 \\
\hline 187 & 16.5 & 0352144 & 0960855 & E & $\mathrm{N}$ & 2,180 & 3,350 & 4,220 & 5,460 & 6,470 & 7,560 & 10,500 \\
\hline 188 & 2.56 & 0351716 & 0960305 & E & $\mathrm{N}$ & 363 & 523 & 637 & 792 & 914 & 1,040 & 1,370 \\
\hline 189 & 105 & 0353850 & 0972135 & E & $\mathrm{N}$ & 6,380 & 11,200 & 14,200 & 17,600 & 19,900 & 21,900 & 25,800 \\
\hline 190 & 532 & 0354051 & 0970029 & E & $\mathrm{R}$ & 11,700 & 19,300 & 25,300 & 34,200 & 41,700 & 50,100 & 73,200 \\
\hline 191 & 46.0 & 0354208 & 0965320 & E & $\mathrm{N}$ & 2,030 & 2,560 & 2,910 & 3,350 & 3,680 & 4,010 & 4,810 \\
\hline
\end{tabular}




\begin{tabular}{|c|c|c|c|c|c|c|c|}
\hline \multirow[b]{2}{*}{$\begin{array}{c}\text { Site } \\
\text { number } \\
\text { (fig. 1) }\end{array}$} & \multirow[b]{2}{*}{$\begin{array}{l}\text { Station } \\
\text { number }\end{array}$} & \multirow[b]{2}{*}{ Station name } & \multirow[b]{2}{*}{$\begin{array}{l}\text { Type of } \\
\text { station } \\
\text { (CONT/ } \\
\text { CSG) }\end{array}$} & \multicolumn{3}{|c|}{ Documented extreme peak discharge } & \multirow[b]{2}{*}{$\begin{array}{l}\text { Potential } \\
\text { extreme } \\
\text { peak } \\
\text { discharge } \\
\left(\mathrm{trth}^{3} / \mathrm{s}\right) \\
\text { (table 4) }\end{array}$} \\
\hline & & & & $\begin{array}{l}\text { Type of } \\
\text { record (H/ } \\
\text { I/// } / \\
\text { R/U) }\end{array}$ & Date & $\begin{array}{c}\text { Discharge } \\
\left(\mathrm{ft}^{3} / \mathrm{s}\right)\end{array}$ & \\
\hline 192 & 07243000 & Dry Creek near Kendrick, Okla. & CONT & $\mathrm{N}$ & $11 / 02 / 74$ & 18,000 & 93,000 \\
\hline 193 & 07243500 & Deep Fork near Beggs, Okla. & CONT & $\mathrm{N}$ & $05 / 11 / 43$ & $66,800^{\mathrm{a}}$ & 273,000 \\
\hline 194 & 07243550 & Adams Creek near Beggs, Okla. & CSG & $\begin{array}{l}\mathrm{R} \\
\mathrm{N}\end{array}$ & $\begin{array}{l}03 / 16 / 90 \\
06 / 08 / 74\end{array}$ & $\begin{array}{r}37,000 \\
3,390\end{array}$ & 28,800 \\
\hline 195 & 07244000 & Deep Fork near Dewar, Okla. & CONT & $\begin{array}{c}\mathrm{HN} \\
\mathrm{N}\end{array}$ & $\begin{array}{l}10 / 0 / 08 \\
04 / 16 / 45\end{array}$ & $\begin{array}{l}85,000 \\
57,400\end{array}$ & 283,000 \\
\hline 196 & 07244790 & Brooken Creek near Enterprise, Okla. & CSG & $\mathrm{N}$ & $05 / 13 / 68$ & 4,200 & 28,000 \\
\hline 197 & 07245000 & Canadian River near Whiteffield, Okla. & CONT & $\begin{array}{l}\mathrm{N} \\
\mathrm{R}\end{array}$ & $\begin{array}{l}05 / 10 / 43 \\
05 / 03 / 90\end{array}$ & $\begin{array}{l}281,000^{h} \\
241,000^{\mathrm{h}}\end{array}$ & 627,000 \\
\hline 198 & 07245090 & Vian Creek near Vian, Okla. & CSG & $\mathrm{N}$ & 04/20/66 & 7,320 & 54,200 \\
\hline 199 & 07245500 & Sallisaw Creek near Sallisaw, Okla. & CONT & $\begin{array}{l}\mathrm{B} \\
\mathrm{N}\end{array}$ & $\begin{array}{l}04 / 15 / 45 \\
102670\end{array}$ & $110,000^{a, b}$ & 132,000 \\
\hline 200 & 07246600 & Cache Creek near Cowlington, Okla. & CSG & $\mathrm{N}$ & $04 / 05 / 64$ & 3,070 & 55,400 \\
\hline 201 & 07246610 & Pecan Creek near Spiro, Okla. & CSG & $\mathrm{N}$ & 05/13/68 & 602 & 5,700 \\
\hline 202 & 07246630 & Big Black Fox Creek near Long, Okla. & CSG & $\mathrm{N}$ & $10 / 26 / 70$ & 1,760 & 26,900 \\
\hline 203 & 07247000 & Poteau River at Cauthron, Ark. & CONT & $\begin{array}{l}\mathrm{N} \\
\mathrm{R}\end{array}$ & $\begin{array}{l}05 / 20 / 60 \\
05 / 03 / 90\end{array}$ & $\begin{array}{l}32,200 \\
24,000\end{array}$ & 137,000 \\
\hline \multirow{2}{*}{204} & 07247500 & Fourche Maline near Red Oak, Okla. & CONT & $\begin{array}{l}\mathrm{R} \\
\mathrm{N}\end{array}$ & $\begin{array}{l}05 / 103 / 90 \\
05 / 19 / 60\end{array}$ & $\begin{array}{l}24,000 \\
41,500\end{array}$ & 114,000 \\
\hline & & & & $\mathrm{R}$ & $11 / 24773$ & 17,800 & \\
\hline \multirow[t]{2}{*}{205} & 07248500 & Poteau River near Wister, Okla. & CONT & $\mathrm{N}$ & $05 / 16 / 45$ & $78,600^{\mathrm{a}}$ & 222,000 \\
\hline & & & & $\begin{array}{c}\mathrm{R} \\
\mathrm{HR}\end{array}$ & $\begin{array}{l}05 / 27 / 57 \\
05 / 00 / 90\end{array}$ & $\begin{array}{l}11,300 \\
25,000^{\mathrm{i}}\end{array}$ & \\
\hline \multirow[t]{2}{*}{206} & 07249000 & Poteau River at Poteau, Okla. & CONT & $\mathrm{HN}$ & $06 / 18 / 35$ & 100,000 & 235,000 \\
\hline & & & & $\mathrm{N}$ & $02 / 19 / 38$ & 73,000 & \\
\hline 207 & 07249300 & James Fork near Midland, Ark. ${ }^{11}$ & CSG & $\mathrm{N}$ & $05 / 14 / 68$ & 25,400 & 77,300 \\
\hline 208 & 07249400 & James Fork near Hackett, Ark. & CONT & $\mathrm{N}$ & $05 / 14 / 68$ & 30,000 & 123,000 \\
\hline 209 & 07249413 & Poteau River near Panama, Okla. & CONT & $\mathrm{R}$ & 05/03/90 & 74,600 & 262,000 \\
\hline 210 & 07249500 & Cove Creek near Lee Creek, Ark. ${ }^{1}$ & CSG & $\mathrm{N}$ & $05 / 05 / 60$ & $33,600^{\mathrm{b}}$ & 70,000 \\
\hline 211 & 07249650 & Mountain Fork near Evansville, A & CSG & $\mathrm{N}$ & $10 / 26 / 70$ & 5,120 & 35,000 \\
\hline 212 & 07249950 & Webber Creek Tributary near Ced & CSG & $\mathrm{N}$ & $10 / 26 / 70$ & 274 & 2,110 \\
\hline \multirow[t]{2}{*}{213} & 07249985 & Lee Creek near Short, Okla. ${ }^{12}$ & CONT & $\mathrm{HN}$ & $\begin{array}{l}04 / 15 / 45 \\
050660\end{array}$ & $\begin{array}{c}112,000^{\mathrm{a}, \mathrm{b}} \\
8060\end{array}$ & 170,000 \\
\hline & & & & $\mathrm{N}$ & 05/06/60 & 80,600 & \\
\hline \multirow[t]{2}{*}{214} & 07250550 & $\begin{array}{l}\text { Arkansas River at James W. Trimble Lock \& Dam } \\
\text { near Van Buren, Ark. }{ }^{13}\end{array}$ & CONT & $\begin{array}{c}\mathrm{N} \\
\mathrm{HN}\end{array}$ & $\begin{array}{l}06 / 19 / 35 \\
05 / 12 / 43\end{array}$ & $\begin{array}{l}418,000 \\
850,000\end{array}$ & 861,000 \\
\hline & & & & $\mathrm{R}$ & 05/05/90 & $401,000^{\mathrm{h}}$ & \\
\hline 215 & 07299540 & Prairie Dog Fork Red River near Childress, Tex. & CONT & $\mathrm{N}$ & $05 / 28 / 78$ & 86,400 & 225,000 \\
\hline 216 & 07299570 & Red River ne: & CON & $\mathrm{N}$ & 05/2: & 73,5 & 231,000 \\
\hline 217 & 07299670 & Groesbeck Creek at State Highway 6 near Quanah, Tex. & CONT & $\mathrm{N}$ & 10/20/83 & 18,000 & 137,000 \\
\hline 218 & 07299705 & Bitter Creek near Hollis, Okla. & CSG & $\mathrm{N}$ & 06/01/68 & 830 & 26,200 \\
\hline \multirow[t]{2}{*}{219} & 07300000 & Salt Fork Red River near Wellington, Tex. ${ }^{14}$ & CONT & $\mathrm{N}$ & 05/16/57 & $146,000^{\mathrm{b}}$ & 187,000 \\
\hline & & & & $\mathrm{R}$ & 04/20/77 & 62,100 & \\
\hline 220 & 07300150 & Bear Creek near Vinson, Okla. & CSG & $\mathrm{N}$ & 05/19/77 & 4,160 & 20,200 \\
\hline 221 & 07300500 & Salt Fork Red River at Magnum, Okla. & CONT & $\mathrm{N}$ & 05/16/57 & $72,000^{\mathrm{a}}$ & 196,000 \\
\hline 222 & 07301110 & Salt Fork Red River near Elmer, Okla. & CONT & $\mathrm{N}$ & 10/20/83 & 44,900 & 204,000 \\
\hline 223 & 07301300 & North Fork Red River near Shamrock, Tex. & CONT & $\mathrm{N}$ & 06/07/67 & 11,200 & 173,000 \\
\hline
\end{tabular}

\begin{tabular}{|c|c|c|c|c|c|c|c|c|c|c|c|c|}
\hline \multirow{2}{*}{$\begin{array}{c}\text { Site } \\
\text { number } \\
\text { (fig. 1) }\end{array}$} & \multirow{2}{*}{$\begin{array}{c}\text { Contrib- } \\
\text { uting } \\
\text { drainage } \\
\text { area }\left(\mathrm{mi}^{2}\right)\end{array}$} & \multirow{2}{*}{ Latitude } & \multirow{2}{*}{ Longitude } & \multirow{2}{*}{$\begin{array}{l}\text { Hydrologic } \\
\text { region (E/ } \\
\text { W) }\end{array}$} & \multirow{2}{*}{$\begin{array}{c}\text { Type } \\
\text { basin (N/ } \\
\text { //R/ } \\
\text { U) }\end{array}$} & \multicolumn{7}{|c|}{$\begin{array}{l}\text { LPIII flood frequency estimates } \\
\text { Peak discharge for indicated recurrence interval (ft } 3 / \mathbf{s})\end{array}$} \\
\hline & & & & & & $2 \mathrm{yr}$ & $5 \mathrm{yr}$ & $10 \mathrm{yr}$ & $25 \mathrm{yr}$ & $50 \mathrm{yr}$ & $100 \mathrm{yr}$ & $500 \mathrm{yr}$ \\
\hline 192 & 69.0 & 0354655 & 0965114 & $E$ & $\mathrm{~N}$ & 3,870 & 6,940 & 9,640 & 13,900 & 17,800 & 22,400 & 36,200 \\
\hline \multirow{2}{*}{193} & 2.018 & 0354026 & 0960406 & $\mathrm{E}$ & $\mathrm{N}$ & 9.440 & 22.600 & 36,900 & 63.800 & 92,100 & 130,000 & 265,000 \\
\hline & & & & & $\mathrm{R}$ & 10,300 & 19,500 & 27,200 & 38,600 & 48,400 & 59,200 & 88,700 \\
\hline 194 & 5.90 & 0354453 & 0960207 & E & $\mathrm{N}$ & 1,090 & 2,100 & 2,980 & 4,360 & 5,590 & 7,010 & 11,200 \\
\hline 195 & 2,307 & 0352843 & 0955257 & $\mathrm{E}$ & $\mathrm{N}$ & 11,100 & 23,600 & 35,600 & 56,000 & 75,400 & 99,200 & 175,000 \\
\hline 196 & 5.66 & 0351450 & 0952250 & E & $\mathrm{N}$ & 1,840 & 3,390 & 4,690 & 6,660 & 8,380 & 10,300 & 15,800 \\
\hline \multirow{2}{*}{197} & 37,876 & 0351550 & 0951421 & E & $\mathrm{N}$ & 100,000 & 175,000 & 233,000 & 315,000 & 382,000 & 453,000 & 640,000 \\
\hline & & & & & $\mathrm{R}$ & 30,300 & 51,400 & 70,800 & 103,000 & 134,000 & 172,000 & 296,000 \\
\hline 198 & 19.6 & 0353214 & 0945815 & E & $\mathrm{N}$ & 2,840 & 4,240 & 5,270 & 6,670 & 7,790 & 8,980 & 12,000 \\
\hline \multirow[t]{2}{*}{199} & 182 & 0352752 & 0945143 & E & $\mathrm{N}$ & 12,700 & 28,500 & 43,900 & 70,100 & 95,200 & 126,000 & 223,000 \\
\hline & & & & & $\mathrm{R}$ & 6,110 & 8,290 & 9,540 & 10,900 & 11,800 & 12,700 & 14,300 \\
\hline 200 & 20.6 & 0351710 & 0944535 & E & $\mathrm{N}$ & 1,600 & 2,120 & 2,470 & 2,920 & 3,260 & 3,610 & 4,440 \\
\hline 201 & 0.90 & 0351440 & 0944435 & E & $\mathrm{N}$ & 265 & 407 & 511 & 654 & 767 & 886 & 1,190 \\
\hline 202 & 5.32 & 0353115 & 0943710 & $\mathrm{E}$ & $\mathrm{N}$ & 865 & 1,410 & 1,810 & 2,370 & 2,800 & 3,260 & 4,430 \\
\hline \multirow[t]{2}{*}{203} & 203 & 0345508 & 0941755 & E & $\mathrm{N}$ & 11,000 & 19,800 & 26,900 & 37,400 & 46,300 & 56,100 & 83,000 \\
\hline & & & & & $\mathrm{R}$ & 9,480 & 13,100 & 15,500 & 18,600 & 21,000 & 23,400 & 29,100 \\
\hline \multirow[t]{2}{*}{204} & 122 & 0345445 & 0950920 & E & $\mathrm{N}$ & 6,560 & 14,100 & 21,200 & 32,900 & 43,800 & 56,900 & 97,300 \\
\hline & & & & & $\mathrm{R}$ & 3,610 & 6,090 & 8,060 & 10,900 & 13,300 & 16,000 & 23,200 \\
\hline \multirow[t]{2}{*}{205} & 993 & 0345615 & 0944254 & E & $\mathrm{N}$ & 25,900 & 51,200 & 73,000 & 107,000 & 137,000 & 170,000 & 267,000 \\
\hline & & & & & $\mathrm{R}$ & 6,560 & 7,740 & 8,500 & 9,420 & 10,100 & 10,800 & 12,300 \\
\hline 206 & 1,240 & 0350335 & 0943610 & E & $\mathrm{N}$ & 22,400 & 46,000 & 67,600 & 103,000 & 135,000 & $\begin{array}{r}172,000 \\
\end{array}$ & 286,000 \\
\hline 207 & 44.0 & 0350427 & 0942020 & $\mathrm{E}$ & $\mathrm{N}$ & 4.570 & 9.440 & 14,000 & 21,600 & 28.700 & 37,200 & 64.000 \\
\hline 208 & 147 & 0350945 & 0942425 & E & $\mathrm{N}$ & 6,590 & 11,100 & 14,700 & 20,000 & 24,300 & 29,200 & 42,300 \\
\hline 209 & 1,767 & 0350956 & 0943910 & E & $\mathrm{R}$ & 22,800 & 39,400 & 52,100 & 69,600 & 83,800 & 98,700 & 137,000 \\
\hline 210 & 35.3 & 0354320 & 0942428 & E & $\mathrm{N}$ & 4,910 & 9,900 & 14,400 & 21,800 & 28,500 & 36,500 & 60,500 \\
\hline 211 & 8.15 & 0354223 & 0942857 & E & $\mathrm{N}$ & 1,240 & 2,450 & 3,470 & 5,040 & 6,390 & 7,920 & 12,200 \\
\hline 212 & 0.34 & 0353600 & 0942249 & E & $\mathrm{N}$ & 34 & 84 & 139 & 239 & 342 & 475 & 937 \\
\hline 213 & 420 & 0353109 & 0942758 & E & $\mathrm{N}$ & 23,900 & 41,200 & 55,000 & 75,100 & 92,000 & 111,000 & 161,000 \\
\hline \multirow{2}{*}{214} & 128,306 & 0352056 & 0941754 & E & $\mathrm{N}$ & 218,000 & 351,000 & 448,000 & 577,000 & 679,000 & 784,000 & $1,040,000$ \\
\hline & & & & & $\mathrm{R}$ & 163,000 & 230,000 & 277,000 & 340,000 & 390,000 & 442,000 & 572,000 \\
\hline 215 & 2,956 & 0343409 & 1001137 & W & $\mathrm{N}$ & 15,800 & 31,200 & 44,200 & 63,600 & 80,300 & 98,800 & 149,000 \\
\hline 216 & 3,552 & 0342447 & 0994403 & W & $\mathrm{N}$ & 20,200 & 35,600 & 47,900 & 65,700 & 80,400 & 96,500 & 139,000 \\
\hline 217 & 303. & 0342116 & 0994424 & W & $\mathrm{N}$ & 1,980 & 5,060 & 8,220 & 13,800 & 19,100 & 25,700 & 46,400 \\
\hline & 10.4 & 0344240 & 0995735 & $\mathrm{w}$ & $\mathrm{N}$ & 129 & 389 & 674 & 1,190 & 1,690 & 2,310 & 4,250 \\
\hline \multirow[t]{2}{*}{219} & 1,013 & 0345727 & 1001314 & w & $\mathrm{N}$ & 18,700 & 39,900 & 60,800 & 97,100 & 133,000 & 177,000 & 326,000 \\
\hline & & & & & $\mathrm{R}$ & 6,960 & 20,500 & 36,300 & 67,300 & 101,000 & 145,000 & 304,000 \\
\hline 220 & 7.24 & 0345401 & 0995854 & $\mathrm{w}$ & $\mathrm{N}$ & 661 & 1,620 & 2,570 & 4,180 & 5,700 & 7,530 & 13,100 \\
\hline 221 & 1,357 & & 0993030 & W & $\mathrm{N}$ & 11,800 & 24,500 & 35,200 & 50,900 & 64,100 & 78,500 & 116,000 \\
\hline 222 & 1,669 & 0342844 & 0992255 & w & $\mathrm{N}$ & 8,970 & 19,300 & 28,700 & 43,700 & 57,200 & 72,700 & 118,000 \\
\hline 223 & 703 & 0351551 & 1001429 & w & $\mathrm{R}$ & 3,500 & 7,430 & 11,300 & 17,900 & 24,400 & 32,400 & 59,000 \\
\hline
\end{tabular}




\begin{tabular}{|c|c|c|c|c|c|c|c|}
\hline \multirow[b]{2}{*}{$\begin{array}{l}\text { Site } \\
\text { number } \\
\text { (fig. 1) }\end{array}$} & \multirow[b]{2}{*}{$\begin{array}{l}\text { Station } \\
\text { number }\end{array}$} & \multirow[b]{2}{*}{ Station name } & \multirow[b]{2}{*}{$\begin{array}{l}\text { Type of } \\
\text { station } \\
\text { (CNNT/ } \\
\text { CSG) }\end{array}$} & \multicolumn{3}{|c|}{ Documented extreme peak discharge } & \multirow[b]{2}{*}{$\begin{array}{c}\text { Potential } \\
\text { extreme } \\
\text { peak } \\
\text { discharge } \\
\left(\mathbf{f t}^{3} / \mathrm{s}\right) \\
\text { (table 4) }\end{array}$} \\
\hline & & & & 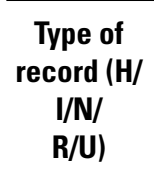 & Date & $\begin{array}{c}\text { Discharge } \\
\left(f \mathrm{tt}^{3} / \mathrm{s}\right)\end{array}$ & \\
\hline 223 & & & & $\mathrm{R}$ & $06 / 03 / 95$ & 25,600 & \\
\hline 224 & 07301410 & Sweetwater Creek near Kelton, Tex. & CONT & $\mathrm{N}$ & 05/20/77 & 2,890 & 131,000 \\
\hline 225 & 07301420 & Sweetwater Creek near Sweetwater, Okla. & CONT & $\begin{array}{l}\mathrm{HN} \\
\mathrm{N}\end{array}$ & $\begin{array}{l}04 / 29 / 54 \\
06 / 03 / 95\end{array}$ & $\begin{array}{r}11,600 \\
1,940\end{array}$ & 151,000 \\
\hline 226 & 07301455 & Turkey Creek near Erick, Okla. & CSG & $\mathrm{N}$ & 00/00/74 & 3,040 & 40,700 \\
\hline 227 & 07301480 & Short Creek near Sayre, Okla. & CSG & $\mathrm{N}$ & 05/19/77 & 2,450 & 24,000 \\
\hline 228 & 07301495 & Indian Creek near Carter, Okla. & CSG & $\mathrm{N}$ & 10/09/68 & 2,350 & 46,400 \\
\hline 229 & 07301500 & North Fork Red River near Carter, Okla. 15 & CONT & $\mathrm{N}$ & 05/26/59 & 53,400 & 210,000 \\
\hline 230 & 07302000 & North Fork Red River near Granite, Okla. & CONT & $\begin{array}{l}\mathrm{HN} \\
\mathrm{N}\end{array}$ & $\begin{array}{l}05 / 18 / 35 \\
04 / 27 / 42\end{array}$ & $\begin{array}{l}28,000 \\
23,900\end{array}$ & 212,000 \\
\hline 231 & 07303000 & $\begin{array}{l}\text { North Fork Red River below Altus Dam } \\
\text { near Lugert, Okla. }\end{array}$ & CONT & $\begin{array}{c}\mathrm{HN} \\
\mathrm{R}\end{array}$ & $\begin{array}{l}05 / 16 / 28 \\
05 / 18 / 51\end{array}$ & $\begin{array}{l}14,300 \\
16,100^{\mathrm{e}}\end{array}$ & 213,000 \\
\hline 232 & 07303400 & Elm Fork of North Fork Red River near Carl, Okla. & CONT & $\mathrm{N}$ & $06 / 13 / 95$ & 62,300 & 151,000 \\
\hline 233 & 07303450 & Deer Creek near Plainview, Okla. & CSG & $\mathrm{N}$ & 06/02/73 & 2,680 & 49,700 \\
\hline 234 & 07303500 & Elm Fork of North Fork Red River near Magnum, Okla. & CONT & $\mathrm{N}$ & $05 / 12 / 47$ & $30,600^{\mathrm{a}}$ & 180,000 \\
\hline 235 & 07304500 & Elk Creek near Hobart, Okla. & CONT & $\begin{array}{l}\mathrm{N} \\
\mathrm{R}\end{array}$ & $\begin{array}{l}10 / 04 / 55 \\
10 / 03 / 86\end{array}$ & $\begin{array}{l}22,400^{\mathrm{a}} \\
28,000\end{array}$ & 163,000 \\
\hline 236 & 07305000 & North Fork Red River near Headrick, Okla. & CONT & $\begin{array}{l}\mathrm{HN} \\
\mathrm{N}\end{array}$ & $\begin{array}{l}05 / 18 / 35 \\
06 / 10 / 41\end{array}$ & $\begin{array}{l}60,000 \\
27,400\end{array}$ & 234,000 \\
\hline 237 & 07305500 & West Otter Ck at Snyder Lk near Mountain Park, Okla. & CONT & $\begin{array}{l}\mathrm{R} \\
\mathrm{R} \\
\mathrm{R}\end{array}$ & $\begin{array}{l}10 / / 04 / 86 \\
06 / 1 / 633 \\
05 / 2 / 87\end{array}$ & $\begin{array}{l}59,000 \\
14,200^{\mathrm{a}} \\
4,300\end{array}$ & 101,000 \\
\hline 238 & 07307028 & North Fork Red River near Tipton, Okla. & CONT & $\mathrm{R}$ & 10/05/86 & 57,200 & 237,000 \\
\hline 239 & 07307800 & Pease River near Childress, Tex. & CONT & $\mathrm{N}$ & 06/05/95 & 28,500 & 214,000 \\
\hline 240 & 07308000 & Pease River near Cromwell, Tex. & CONT & $\mathrm{N}$ & 06/06/41 & $106,000^{\mathrm{a}}$ & 218,000 \\
\hline 241 & 07308200 & Pease River near Vernon, Tex. & CONT & $\mathrm{N}$ & 10/02/83 & 40,500 & 225,000 \\
\hline 242 & 07308500 & Red River near Burkburnett, Tex. & CONT & $\mathrm{N}$ & 06/06/95 & 174,000 & 275,000 \\
\hline 243 & 07309480 & Canyon Creek near Medicine Park, Okla. & CSG & $\mathrm{N}$ & 07/03/67 & 2,200 & 11,200 \\
\hline 244 & 07311000 & East Cache Creek near Walters, Okla. & CONT & $\begin{array}{l}\mathrm{N} \\
\mathrm{R}\end{array}$ & $\begin{array}{l}05 / 18 / 51 \\
10 / 21 / 83\end{array}$ & $\begin{array}{l}28,200^{\mathrm{a}, \mathrm{e}} \\
50,900\end{array}$ & 172,000 \\
\hline 245 & 07311200 & Blue Beaver Creek near Cache, Okla. & CONT & $\mathrm{N}$ & 08/28/77 & $13,600^{\mathrm{j}}$ & 46,100 \\
\hline 246 & 07311410 & Red Creek near Snyder, Okla. & CSG & $\mathrm{N}$ & $07 / 01 / 68$ & 800 & 17,700 \\
\hline 247 & 07311420 & Deadman Creek Tributary at Manitou, Okla. & CSG & $\mathrm{N}$ & $07 / 13 / 68$ & 980 & 9,010 \\
\hline 248 & 07311500 & Deep Red Creek near Randlett, Okla. & CONT & $\mathrm{N}$ & 10/20/83 & 72,300 & 168,000 \\
\hline 249 & 07312200 & Beaver Creek near Electra, Tex. & CONT & $\mathrm{R}$ & 03/17/61 & 11,700 & 170,000 \\
\hline 250 & 07312500 & Wichita River at Wichita Falls, Tex. & CONT & $\begin{array}{c}\mathrm{HN} \\
\mathrm{R}\end{array}$ & $\begin{array}{l}05 / 08 / 15 \\
10 / 03 / 41\end{array}$ & $\begin{array}{l}50,000 \\
17,800\end{array}$ & 188,000 \\
\hline 251 & 07312850 & Nine Mile Beaver Creek near Elgin, Okla. & CSG & $\mathrm{N}$ & 10/20/83 & $3,950^{\mathrm{g}}$ & 18,100 \\
\hline 252 & 07312950 & Little Beaver Creek near Marlow, Okla. & CSG & $\mathrm{N}$ & $05 / 31 / 68$ & 6,400 & 70,100 \\
\hline 253 & 07313000 & Little Beaver Creek near Duncan, Okla. 16 & CONT & $\mathrm{N}$ & $05 / 25 / 57$ & $47,500^{\mathrm{a}}$ & 128,000 \\
\hline 254 & 07313500 & Beaver Creek near Waurika, Okla. & CONT & $\begin{array}{l}\mathrm{HN} \\
\mathrm{N}\end{array}$ & $\begin{array}{l}05 / 18 / 51 \\
05 / 20 / 55\end{array}$ & $\begin{array}{l}65,300^{\mathrm{a}} \\
32,200\end{array}$ & 187,000 \\
\hline & & & & $\mathrm{R}$ & $06 / 109 / 87$ & 2,710 & \\
\hline 255 & 07313600 & Cow Creek near Waurika, Okla. ${ }^{1}$ & CSG & $\begin{array}{c}\mathrm{HN} \\
\mathrm{N}\end{array}$ & $\begin{array}{l}05 / 19 / 55 \\
05 / 24 / 75\end{array}$ & $\begin{array}{r}29,500 \\
7,420\end{array}$ & 134,000 \\
\hline
\end{tabular}

\begin{tabular}{|c|c|c|c|c|c|c|c|c|c|c|c|c|}
\hline \multirow{2}{*}{$\begin{array}{c}\text { Site } \\
\text { number } \\
\text { (fig. 1) }\end{array}$} & \multirow{2}{*}{$\begin{array}{c}\text { Contributing } \\
\text { drainage area } \\
\left(\mathrm{mi}^{2}\right)\end{array}$} & \multirow{2}{*}{ Latitude } & \multirow{2}{*}{ Longitude } & \multirow{2}{*}{$\begin{array}{c}\text { Hydrologic } \\
\text { region (E/ } \\
\text { W) }\end{array}$} & \multirow{2}{*}{$\begin{array}{c}\text { Type } \\
\text { basin (N/ } \\
\text { I/R/ } \\
\text { U) }\end{array}$} & \multicolumn{7}{|c|}{$\begin{array}{l}\text { LPIII flood frequency estimates } \\
\text { Peak discharge for indicated recurrence interval (ftr } 3 / \mathrm{s})\end{array}$} \\
\hline & & & & & & $2 \mathrm{yr}$ & $5 \mathrm{yr}$ & $10 \mathrm{yr}$ & $25 \mathrm{yr}$ & $50 \mathrm{yr}$ & $100 \mathrm{yr}$ & $500 \mathrm{yr}$ \\
\hline 224 & 267 & 0352823 & 1000714 & $\mathrm{~W}$ & $\mathrm{~N}$ & 465 & 1,010 & 1,520 & 2,320 & 3,060 & 3,900 & 6,380 \\
\hline 225 & 424 & 0352520 & 0995808 & W & $\mathrm{N}$ & 263 & 680 & 1,180 & 2,200 & 3,370 & 5,020 & 11,800 \\
\hline 226 & 19.8 & 0351205 & 0994755 & $\mathrm{w}$ & $\mathrm{N}$ & 1,020 & 1,940 & 2,720 & 3,910 & 4,940 & 6,110 & 9,410 \\
\hline 227 & 9.12 & 0351820 & 0993915 & $\mathrm{w}$ & $\mathrm{N}$ & 441 & 923 & 1,386 & 2,170 & 2,930 & 3,860 & 6,860 \\
\hline 228 & 24.9 & 0351730 & 0993035 & $\mathrm{w}$ & $\mathrm{N}$ & 724 & 1,330 & 1,850 & 2,660 & 3,380 & 4,200 & 6,600 \\
\hline 229 & 1,938 & 0351005 & 0993025 & w & $\mathrm{N}$ & 6,540 & 14,300 & 21,200 & 31,800 & 41,000 & 51,300 & 79,500 \\
\hline 230 & 2,095 & 0345824 & 0992000 & $\mathrm{w}$ & $\mathrm{N}$ & 9,320 & 15,300 & 19,700 & 25,600 & 30,200 & 34,900 & 46,700 \\
\hline 231 & 2,116 & 0345322 & 0991824 & w & $\mathrm{R}$ & 155 & 2,760 & 7,570 & 16,300 & 23,300 & 29,800 & 41,000 \\
\hline 232 & 416 & 0350042 & 0995412 & W & $\mathrm{N}$ & 4,980 & 10,100 & 15,100 & 23,500 & 31,600 & 41,500 & 73,800 \\
\hline 233 & 27.8 & 0350250 & 0994610 & $\mathrm{w}$ & $\mathrm{N}$ & 929 & 1,660 & 2,250 & 3,110 & 3,830 & 4,630 & 6,760 \\
\hline 234 & 838 & 0345536 & 0993000 & W & $\mathrm{N}$ & 7,400 & 15,000 & 21,100 & 29,800 & 36,900 & 44,400 & 63,500 \\
\hline \multirow[t]{2}{*}{235} & 549 & 0345451 & 0990649 & w & $\mathrm{N}$ & 4,080 & 6,930 & 9,150 & 12,300 & 14,900 & 17,700 & 25,200 \\
\hline & & & & & $\mathrm{R}$ & 5,610 & 11,700 & 17,400 & 27,100 & 36,200 & 47,200 & 82,000 \\
\hline \multirow[t]{2}{*}{236} & 3,845 & 0343804 & 0990547 & W & $\mathrm{N}$ & 17,600 & 28,000 & 35,900 & 47,300 & 56,600 & 66,800 & 94,000 \\
\hline & & & & & $\mathrm{R}$ & 12,300 & 22,800 & 31,000 & 42,800 & 52,500 & 62,900 & 89,900 \\
\hline 237 & 132 & 0344402 & 0985910 & w & $\mathrm{R}$ & 145 & 440 & 813 & 1,610 & 2,540 & 3,860 & 9,270 \\
\hline 238 & 4,292 & 0343025 & 0991228 & w & $\mathrm{R}$ & 12,600 & 20,800 & 27.800 & 38,600 & 48,400 & 59,800 & 94,200 \\
\hline 239 & 2,195 & 0341339 & 1000424 & w & $\mathrm{N}$ & 6,550 & 11,600 & 15,500 & 20,800 & 25,100 & 29,600 & 40,900 \\
\hline 240 & 2,478 & 0340545 & 0994347 & w & $\mathrm{N}$ & 39,400 & 64,700 & 82,300 & 105,000 & 121,000 & 138,000 & 176,000 \\
\hline 241 & 2,929 & 0341045 & 0991640 & W & $\mathrm{N}$ & 10,300 & 20,700 & 28,900 & 40,200 & 49,100 & 58,300 & 80,600 \\
\hline 242 & 14,634 & 0340636 & 0983153 & $\mathrm{w}$ & $\mathrm{N}$ & 30,900 & 60,300 & 84,600 & 121,000 & 151,000 & 185,000 & 276,000 \\
\hline 243 & 3.35 & 0344955 & 0983210 & w & $\mathrm{N}$ & 829 & 1,930 & 2,900 & 4,370 & 5,620 & 6,980 & 10,600 \\
\hline \multirow{2}{*}{244} & 675 & 0342144 & 0981656 & W & $\mathrm{N}$ & 7,450 & 12,500 & 16,400 & 21,800 & 26,300 & 31,200 & 44,000 \\
\hline & & & & & $\mathrm{R}$ & 5,570 & 12,400 & 19,100 & 30,600 & 41,800 & 55,500 & 99,700 \\
\hline 245 & 24.6 & 0343724 & 0983348 & w & $\mathrm{N}$ & 1,780 & 3,650 & 5,260 & 7,720 & 9,860 & 12,200 & 18,900 \\
\hline 246 & 6.12 & 0344058 & 0985140 & W & $\mathrm{N}$ & 142 & 344 & 533 & 831 & 1,090 & 1,390 & 2,220 \\
\hline 247 & 2.57 & 0343017 & 0985901 & w & $\mathrm{N}$ & 373 & 780 & 1,130 & 1,640 & 2,080 & 2,560 & 3,850 \\
\hline 248 & 617 & 0341315 & 0982710 & w & $\mathrm{N}$ & 7,850 & 17,200 & 25,700 & 39,300 & 51,600 & 65,800 & 107,000 \\
\hline 249 & 652 & 0335421 & 0985417 & w & $\mathrm{R}$ & 2,880 & 5,050 & 6,840 & 9,530 & 11,800 & 14,400 & 21,800 \\
\hline 250 & 1,054 & 0335434 & 0983200 & w & $\mathrm{R}$ & 4,130 & 6,910 & 8,800 & 11,200 & 12,900 & 14,600 & 18,300 \\
\hline 251 & 6.29 & 0344640 & 0981525 & W & $\mathrm{N}$ & 730 & 1,790 & 2,860 & 4,710 & 6,510 & 8,700 & 15,700 \\
\hline 252 & 35.4 & 0344057 & 0980031 & E & $\mathrm{N}$ & 807 & 2,000 & 3,330 & 5,880 & 8,600 & 12,200 & 25,700 \\
\hline 253 & 158 & 0342935 & 0980650 & E & $\mathrm{N}$ & 14,100 & 26,700 & 36,700 & 51,100 & 62,900 & 75,600 & 109,000 \\
\hline \multirow{2}{*}{254} & 563 & 0341300 & 0980257 & E & $\mathrm{N}$ & 4,030 & 10,200 & 17,100 & 30,800 & 45,900 & 66,200 & 144,000 \\
\hline & & & & & $\mathrm{R}$ & 1,660 & 2,600 & 2,920 & 3,130 & 3,200 & 3,240 & 3,270 \\
\hline \multirow[t]{2}{*}{255} & 193 & 0341055 & 0980005 & E & $\mathrm{N}$ & 2,260 & 5,760 & 9,520 & 16,400 & 23,500 & 32,600 & 64,000 \\
\hline & & & & & $\mathrm{R}$ & 3,520 & 7,880 & 12,000 & 18,800 & 25,200 & 32,700 & 55,500 \\
\hline
\end{tabular}




\begin{tabular}{|c|c|c|c|c|c|c|c|}
\hline \multirow[b]{2}{*}{$\begin{array}{c}\text { Site } \\
\text { number } \\
\text { (fig. 1) }\end{array}$} & \multirow[b]{2}{*}{$\begin{array}{l}\text { Station } \\
\text { number }\end{array}$} & \multirow[b]{2}{*}{ Station name } & \multirow[b]{2}{*}{$\begin{array}{l}\text { Type of } \\
\text { station } \\
\text { (CONT/ } \\
\text { CSG) }\end{array}$} & \multicolumn{3}{|c|}{ Documented extreme peak discharge } & \multirow[b]{2}{*}{$\begin{array}{c}\text { Potential } \\
\text { extreme } \\
\text { peak } \\
\text { discharge } \\
\left(\mathrm{ftt}^{3} / \mathrm{s}\right) \\
(\text { table 4) }\end{array}$} \\
\hline & & & & $\begin{array}{c}\text { Type of } \\
\text { record (H/ } \\
\text { I/N/ } \\
\text { R/U) }\end{array}$ & Date & $\begin{array}{c}\text { Discharge } \\
\left(\mathrm{ft}^{3} / \mathrm{s}\right)\end{array}$ & \\
\hline 255 & & & & $\mathrm{R}$ & 10/20/83 & $18,100^{g}$ & \\
\hline 256 & 07314900 & Little Witchita River above Henrietta, Tex. & CONT & $\begin{array}{l}R \\
R\end{array}$ & $\begin{array}{l}05 / 01 / 66 \\
05 / 03 / 90\end{array}$ & $\begin{array}{c}7,630 \\
14,200^{\mathrm{h}}\end{array}$ & 224,000 \\
\hline 257 & 07315200 & East Fork Little Wichita River near Henrietta, Tex. & CONT & $\mathrm{N}$ & $10 / 13 / 81$ & $32,500^{\mathrm{k}}$ & 131,000 \\
\hline 258 & 07315500 & Red River near Terral, Okla. & CONT & $\begin{array}{l}\mathrm{N} \\
\mathrm{R}\end{array}$ & $\begin{array}{l}06 / 06 / 41 \\
06 / 07 / 95\end{array}$ & $\begin{array}{l}197,000 \\
236,000\end{array}$ & 546,000 \\
\hline 259 & 07315680 & Cottonwood Creek Tributary near Loco, Okla. & CSG & $\mathrm{N}$ & $06 / 22 / 65$ & 2,100 & 10,600 \\
\hline 260 & 07315700 & Mud Creek near Courtney, Okla. & CONT & $\begin{array}{c}\mathrm{HN} \\
\mathrm{N}\end{array}$ & $\begin{array}{l}05 / 00 / 57 \\
05 / 03 / 90\end{array}$ & $\begin{array}{l}30,000 \\
49,600\end{array}$ & 188,000 \\
\hline 261 & 07315880 & Demijohn Creek near Wilson, Okla. & CSG & $\begin{array}{c}\mathrm{N} \\
\mathrm{HN}\end{array}$ & $\begin{array}{l}04 / 12 / 67 \\
10 / 00 / 81\end{array}$ & $\begin{array}{l}2,300 \\
6,040\end{array}$ & 28,200 \\
\hline 262 & 07316000 & Red River near Gainesville, Tex. & CONT & $\begin{array}{l}\mathrm{N} \\
\mathrm{R}\end{array}$ & $\begin{array}{l}06 / 09 / 41 \\
05 / 31 / 87\end{array}$ & $\begin{array}{l}168,000 \\
265,000\end{array}$ & 559,000 \\
\hline 263 & 07316130 & Wilson Creek near McMillan, Okla. & CSG & $\begin{array}{c}\mathrm{N} \\
\mathrm{HN}\end{array}$ & $\begin{array}{l}05 / 23 / 75 \\
10 / 00 / 81\end{array}$ & $\begin{array}{l}1,380 \\
1,980 \mathrm{k}\end{array}$ & 17,100 \\
\hline 264 & 07316140 & Brier Creek near Powell, Okla. & CSG & $\mathrm{N}$ & $10 / 14 / 81$ & $14,100^{\mathrm{k}}$ & 42,900 \\
\hline 265 & 07316500 & Washita River near Cheyenne, Okla. & CONT & $\begin{array}{c}\mathrm{HN} \\
\mathrm{N} \\
\mathrm{R}\end{array}$ & $\begin{array}{l}04 / 03 / 34 \\
04 / 29 / 54 \\
04 / 22 / 90\end{array}$ & $\begin{array}{r}52,000 \\
69,800 \\
7250\end{array}$ & 178,000 \\
\hline & & & & $\mathrm{R}$ & $04 / 22 / 90$ & 7,250 & \\
\hline 266 & 07317500 & Sandstone Creek SWS 16A near Cheyenne, Okla. ${ }^{17}$ & CONT & $\mathrm{N}$ & $05 / 26 / 59$ & 2,710 & 23,300 \\
\hline 267 & 07318000 & Sandstone Creek SWS 16 near Cheyenne, Okla. ${ }^{17}$ & CONT & $\mathrm{N}$ & $05 / 23 / 54$ & 18,900 & 41,700 \\
\hline 268 & 07318500 & Sandstone Creek SWS 14 near Cheyenne, Okla. ${ }^{17}$ & CONT & $\mathrm{N}$ & $04 / 18 / 57$ & 1,160 & 4,090 \\
\hline 269 & 07319000 & Sandstone Creek SWS 17 near Cheyenne, Okla. ${ }^{17}$ & CONT & $\mathrm{N}$ & $04 / 29 / 54$ & 6,030 & 25,700 \\
\hline 270 & 07319500 & Sandstone Creek near Berlin, Okla. & CONT & $\mathrm{R}$ & $04 / 30 / 54$ & 5,710 & 63,200 \\
\hline 271 & 07320000 & Sandstone Creek SWS 10A near Elk City, Okla. ${ }^{17}$ & CONT & $\mathrm{N}$ & $08 / 16 / 68$ & 1,700 & 9,910 \\
\hline 272 & 07320500 & Sandstone Creek SWS 6 near Elk City, Okla. ${ }^{17}$ & CONT & $\mathrm{N}$ & $05 / 03 / 57$ & 1,870 & 18,500 \\
\hline 273 & 07321000 & Sandstone Creek SWS 5 near Elk City, Okla. ${ }^{17}$ & CONT & $\mathrm{N}$ & $08 / 16 / 68$ & 2,850 & 12,600 \\
\hline 274 & 07321500 & Sandstone Creek SWS 3 near Elk City, Okla. ${ }^{17}$ & CONT & $\mathrm{N}$ & $04 / 18 / 57$ & 1,780 & 2,580 \\
\hline 275 & 07322000 & Sandstone Creek SWS 9 near Elk City, Okla. ${ }^{17}$ & CONT & $\mathrm{N}$ & 06/08/71 & 2,420 & 11,600 \\
\hline 276 & 07323000 & Sandstone Creek near Cheyenne, Okla. & CONT & $\mathrm{R}$ & $04 / 30 / 54$ & 6,360 & 85,200 \\
\hline 277 & 07324000 & Sandstone Creek SWS 1 near Cheyenne, Okla. ${ }^{17}$ & CONT & $\mathrm{N}$ & $04 / 18 / 57$ & 4,280 & 15,900 \\
\hline 278 & 07324200 & Washita River near Hammon, Okla. & CONT & $\begin{array}{l}\mathrm{H} \\
\mathrm{R}\end{array}$ & $\begin{array}{l}04 / 03 / 34 \\
05 / 17 / 82\end{array}$ & $\begin{array}{l}167,000^{1} \\
60000^{2}\end{array}$ & 197,000 \\
\hline 279 & 07324400 & Washita River near Foss, Okla. & CONT & $\begin{array}{l}\mathrm{R} \\
\mathrm{N}\end{array}$ & $\begin{array}{l}05 / 17 / 82 \\
04 / 19 / 57\end{array}$ & $\begin{array}{r}6,000 \\
14,000\end{array}$ & 201,000 \\
\hline & & & & $\mathrm{R}$ & $08 / 26 / 69$ & 3,010 & \\
\hline 280 & 07325000 & Washita River near Clinton, Okla. & CONT & $\begin{array}{c}\mathrm{HN} \\
\mathrm{N}\end{array}$ & $\begin{array}{l}04 / 03 / 34 \\
05 / 16 / 51\end{array}$ & $\begin{array}{l}90,000 \\
66,800^{\mathrm{e}}\end{array}$ & 210,000 \\
\hline & & & & $\mathrm{R}$ & 09/15/96 & 10,800 & \\
\hline 281 & 07325500 & Washita River at Carnegie, Okla. & CONT & $\mathrm{N}$ & $05 / 18 / 49$ & 50,000 & 227,000 \\
\hline & & & & $\mathrm{R}$ & 10/20/83 & 40,600 & \\
\hline 282 & 07325800 & Cobb Creek near Eakly, Okla. & CONT & $\mathrm{R}$ & 06/04/95 & 12,000 & 101,000 \\
\hline 283 & 07325850 & Lake Creek near Eakly, Okla. & CONT & $\mathrm{N}$ & 05/20/77 & 7,000 & 67,600 \\
\hline 284 & 07326000 & Cobb Creek near Fort Cobb, Okla. & CONT & $\mathrm{HN}$ & $06 / 15 / 37$ & 51,000 & 138,000 \\
\hline & & & & $\mathrm{N}$ & $05 / 17 / 49$ & $35,000^{\mathrm{a}}$ & \\
\hline & & & & $\mathrm{R}$ & 06/23/87 & 1,280 & \\
\hline 285 & 07326500 & Washita River at Anadarko, Okla. & CONT & $\mathrm{N}$ & $05 / 25 / 03$ & 29,000 & 326,000 \\
\hline
\end{tabular}

$\begin{array}{cccccccccccc}\text { Site } & \begin{array}{c}\text { Contributing } \\ \text { number } \\ \text { (fig. 1) }\end{array} & \begin{array}{l}\text { drainage area } \\ \left(\mathrm{mi}^{2}\right)\end{array}\end{array}$

\begin{tabular}{|c|c|c|c|c|c|c|c|c|c|c|c|c|}
\hline 256 & 1,037 & 0334936 & 0981423 & E & $\mathrm{R}$ & 1,280 & 3,260 & 5,050 & 7,760 & 10,000 & 12,500 & 18,800 \\
\hline 257 & 178 & 0334846 & 0980505 & E & $\mathrm{N}$ & 1,720 & 5,360 & 9,800 & 18,800 & 28,800 & 42,400 & 93,400 \\
\hline 258 & 22,787 & 0335243 & 0975603 & E & $\mathrm{R}$ & 45,200 & 85,300 & 121,000 & 176,000 & 227,000 & 286,000 & 463,000 \\
\hline 259 & 1.74 & 0341840 & 0973400 & E & $\mathrm{N}$ & 483 & 1,080 & 1,670 & 2,700 & 3,690 & 4,920 & 8,910 \\
\hline 260 & 572 & 0340015 & 0973400 & E & $\mathrm{N}$ & 6,260 & 16,200 & 27,300 & 48,400 & 70,800 & 100,000 & 206,000 \\
\hline 261 & 5.74 & 0340810 & 0972520 & E & $\mathrm{N}$ & 1,950 & 2,570 & 3,020 & 3,630 & 4,120 & 4,640 & 5,970 \\
\hline 262 & 24,846 & 0334340 & 0970935 & $\mathrm{E}$ & R & 49,600 & 88,800 & 120,000 & 165,000 & 202,000 & 243,000 & 352,000 \\
\hline 263 & 2.97 & 0340600 & 0965835 & E & $\mathrm{N}$ & 760 & 1,100 & 1,360 & 1,700 & 1,970 & 2,260 & 3,000 \\
\hline 264 & 12.0 & 0335954 & 0964935 & E & $\mathrm{N}$ & 2,870 & 5,430 & 7,740 & 11,500 & 14,900 & 19,000 & 31,500 \\
\hline \multirow[t]{2}{*}{265} & 794 & 0353735 & 0994005 & w & $\mathrm{N}$ & 5,500 & 15,400 & 26,900 & 49,200 & 73,200 & 105,000 & 222,000 \\
\hline & & & & & $\mathrm{R}$ & 696 & 2,010 & 3,540 & 6,570 & 9,850 & 14,200 & 30,400 \\
\hline 266 & 8.78 & 0352810 & 0994010 & W & $\mathrm{N}$ & 438 & 1,060 & 1,720 & 2,940 & 4,200 & 5,830 & 11,500 \\
\hline 267 & 20.3 & 0352840 & 0993610 & W & $\mathrm{R}$ & 1,670 & 3,910 & 6,340 & 10,900 & 15,700 & 22,100 & 45,400 \\
\hline 268 & 1.02 & 0352840 & 0993610 & W & $\mathrm{N}$ & 290 & 681 & 1,070 & 1,740 & 2,400 & 3,200 & 5,770 \\
\hline 269 & 10.1 & 0353030 & 0993640 & W & $\mathrm{N}$ & 960 & 2,370 & 3,800 & 6,300 & 8,710 & 11,700 & 21,100 \\
\hline 270 & 44.9 & 0353026 & 0993327 & W & $\mathrm{R}$ & 670 & 1,540 & 2,440 & 4,080 & 5,760 & 7,910 & 15,400 \\
\hline 271 & 2.87 & 0352800 & 0993320 & $\mathrm{w}$ & $\mathrm{N}$ & 742 & 1,250 & 1,640 & 2,220 & 2.650 & 3,130 & 4,410 \\
\hline 272 & 6.46 & 0352910 & 0993010 & W & $\mathrm{R}$ & 592 & 1,210 & 1,750 & 2,580 & 3,320 & 4,150 & 6,520 \\
\hline 273 & 3.89 & 0352930 & 0992920 & W & $\mathrm{R}$ & 977 & 1,750 & 2,260 & 2,870 & 3,290 & 3,680 & 4,490 \\
\hline 274 & 0.62 & 0353040 & 0993040 & $\mathrm{w}$ & $\mathrm{N}$ & 349 & 684 & 974 & 1,420 & 1,810 & 2,250 & 3,500 \\
\hline 275 & 3.50 & 0352940 & 0993200 & W & $\mathrm{N}$ & 813 & 1,530 & 2,140 & 3,080 & 3,900 & 4,820 & 7,470 \\
\hline 276 & 87.1 & 0353310 & 0993150 & W & $\mathrm{R}$ & 1,250 & 2,520 & 3,560 & 5,060 & 6,300 & 7,610 & 11,000 \\
\hline 277 & 5.33 & 0353400 & 0993010 & W & $\mathrm{N}$ & 994 & 2,320 & 3,640 & 5,920 & 8,120 & 10,800 & 19,400 \\
\hline 278 & 1,387 & 0353923 & 0991821 & W & $\mathrm{R}$ & 1,040 & 2,240 & 3,290 & 4,900 & 6,280 & 7,820 & 12,000 \\
\hline 279 & 1,551 & 0353220 & 0991010 & W & $\mathrm{R}$ & 864 & 1,450 & 1,860 & 2,360 & 2,740 & 3,100 & 3,900 \\
\hline \multirow{2}{*}{280} & 1,977 & 0353151 & 0985800 & $\mathrm{w}$ & $\mathrm{N}$ & 7,800 & 18,200 & 29,200 & 49,700 & 71,000 & 98,900 & 199,000 \\
\hline & & & & & $\mathrm{R}$ & 2,090 & 4,090 & 6,000 & 9,270 & 12,400 & 16,400 & 29,300 \\
\hline \multirow[t]{2}{*}{281} & 3,129 & 0350702 & 0983349 & W & $\mathrm{N}$ & 9,210 & 17,000 & 23,600 & 33,700 & 42,600 & 52,700 & 81,500 \\
\hline & & & & & $\mathrm{R}$ & 5,740 & 12,000 & 18,500 & 30,000 & 41,900 & 57,200 & 111,000 \\
\hline 282 & 132 & 0351726 & 0983538 & w & $\mathrm{R}$ & 2,060 & 4,490 & 7,060 & 11,800 & 16,800 & 23,300 & 47,000 \\
\hline 283 & 52.0 & 0351727 & 0983144 & $\mathrm{w}$ & $\mathrm{N}$ & 707 & 1,850 & 3,160 & 5,740 & 8,560 & 12,400 & 26,900 \\
\hline \multirow[t]{2}{*}{284} & 307 & 0350837 & 0982633 & W & $\mathrm{N}$ & 4,420 & 10,500 & 16,900 & 28,700 & 40,800 & 56,500 & 112,000 \\
\hline & & & & & $\mathrm{R}$ & 535 & 1,020 & 1,340 & 1,680 & 1,90 & 2,10 & 2,440 \\
\hline 285 & 3.656 & 0350503 & 0981435 & E & $\mathrm{N}$ & 8,720 & 18.300 & 27,400 & 42,600 & 57,200 & 74,700 & 130,000 \\
\hline
\end{tabular}




\begin{tabular}{|c|c|c|c|c|c|c|c|}
\hline \multirow[b]{2}{*}{$\begin{array}{c}\text { Site } \\
\text { number } \\
\text { (fig. 1) }\end{array}$} & \multirow[b]{2}{*}{$\begin{array}{l}\text { Station } \\
\text { number }\end{array}$} & \multirow[b]{2}{*}{ Station name } & \multirow[b]{2}{*}{$\begin{array}{l}\text { Type of } \\
\text { station } \\
\text { (CNONT/ } \\
\text { CSG) }\end{array}$} & \multicolumn{3}{|c|}{ Documented extreme peak discharge } & \multirow[b]{2}{*}{$\begin{array}{c}\text { Potentiala } \\
\text { extreme } \\
\text { peak } \\
\text { discharge } \\
\text { (ttit/s) } \\
\text { (table 4) }\end{array}$} \\
\hline & & & & $\begin{array}{l}\text { Type of } \\
\text { record (H/ } \\
\text { I/N/ } \\
\text { R/U) }\end{array}$ & Date & $\begin{array}{c}\text { Discharge } \\
\left(\mathrm{ft}^{3} / \mathrm{s}\right)\end{array}$ & \\
\hline 285 & & & & $\begin{array}{c}\mathrm{HN} \\
\mathrm{R}\end{array}$ & $\begin{array}{l}05 / 18 / 49 \\
06 / 06 / 95\end{array}$ & $\begin{array}{l}45,000 \\
52,800\end{array}$ & \\
\hline 286 & 07327000 & Sugar Creek near Gracemont, Okla. & CONT & $\begin{array}{l}\mathrm{HN} \\
\mathrm{N} \\
\mathrm{R}\end{array}$ & $\begin{array}{l}05 / 17 / 49 \\
10 / 04 / 59 \\
09 / 21 / 65\end{array}$ & $\begin{array}{r}32,000 \\
1,260 \\
8,500\end{array}$ & 121,000 \\
\hline 287 & 07327040 & Delaware Creek Number 131 near Anadarko, Okla. & CONT & $\mathrm{R}$ & $05 / 31 / 77$ & 1,201 & 74,200 \\
\hline 288 & 07327150 & Salt Creek near Chickasha, Okla. & CONT & $\mathrm{N}$ & 04/12/67 & 4,900 & 58,800 \\
\hline 289 & 07327210 & Line Creek Number 411 at Chickasha, Okla. & CONT & $\mathrm{N}$ & 08/28/65 & 2,010 & 83,200 \\
\hline 290 & 07327420 & West Bitter Creek near Tabler, Okla. & CONT & $\mathrm{N}$ & 04/12/67 & 3,300 & 87,600 \\
\hline 291 & 07327439 & Watershed R-7 near Tabler, Okla. & CONT & $\mathrm{N}$ & 00/00/73 & 98 & 1,830 \\
\hline 292 & 07327440 & East Bitter Creek near Tabler, Okla. & CONT & $\mathrm{R}$ & $05 / 24 / 73$ & 4,620 & 69,900 \\
\hline \multirow[t]{4}{*}{293} & 07327490 & Little Washita River near Ninnekah, Okla. ${ }^{18}$ & CONT & HN & $05 / 16 / 47$ & 36,000 & 137,000 \\
\hline & & & & $\mathrm{HN}$ & $05 / 24 / 57$ & 25,200 & \\
\hline & & & & $\mathrm{N}$ & 05/10/64 & 9,360 & \\
\hline & & & & $\mathrm{R}$ & 10/20/83 & $9,380^{\mathrm{g}}$ & \\
\hline \multirow[t]{3}{*}{294} & 07328000 & Washita River near Tabler, Okla. & CONT & $\mathrm{HN}$ & 04/07/27 & 53,600 & 353,000 \\
\hline & & & & $\mathrm{N}$ & 05/20/49 & 50,000 & \\
\hline & & & & $\mathrm{HN}$ & 05/00/57 & 48,300 & \\
\hline 295 & 07328030 & Big Dry Creek near Alex, Okla. & CSG & $\mathrm{R}$ & 05/09/64 & 2,450 & 33,600 \\
\hline 296 & 07328040 & Little Dry Creek near Alex, Okla. & CSG & $\mathrm{R}$ & 07/01/68 & 280 & 5,580 \\
\hline 297 & 07328070 & Winter Creek near Alex, Okla. & CONT & $\mathrm{R}$ & $05 / 27 / 78$ & 6,080 & 68,000 \\
\hline 298 & 07328100 & Washita River at Alex, Okla. & CONT & $\mathrm{R}$ & 06/08/95 & 25,000 & 355,000 \\
\hline \multirow[t]{2}{*}{299} & 07328500 & Washita River near Pauls Valley, Okla. & CONT & $\mathrm{N}$ & $05 / 18 / 57$ & 35,800 & 366,000 \\
\hline & & & & $\mathrm{R}$ & 05/29/87 & 43,600 & \\
\hline \multirow[t]{2}{*}{300} & 07329000 & Rush Creek at Purdy, Okla. & CONT & $\mathrm{N}$ & 05/10/50 & 30,000 & 122,000 \\
\hline & & & & $\mathrm{R}$ & $05 / 28 / 87$ & 17,000 & \\
\hline \multirow[t]{2}{*}{301} & 07329500 & Rush Creek near Maysville, Okla. ${ }^{19}$ & CONT & $\mathrm{N}$ & $05 / 18 / 57$ & 38,500 & 137,000 \\
\hline & & & & $\mathrm{R}$ & 05/21/79 & 17,600 & \\
\hline 302 & 07329700 & Wild Horse Creek near Hoover, Okla. & CONT & $\mathrm{R}$ & 05/03/90 & 40,600 & 191,000 \\
\hline 303 & 07329810 & Honey Creek near Davis, Okla. & CSG & $\mathrm{N}$ & $10 / 13 / 81$ & $16,200^{\mathrm{k}}$ & 53,000 \\
\hline 304 & 07329852 & Rock Creek at Sulphur, Okla. & CONT & $\mathrm{R}$ & 04/26/90 & 10,400 & 77,300 \\
\hline \multirow[t]{2}{*}{305} & 07329900 & Rock Creek near Dougherty, Okla. & CONT & $\mathrm{N}$ & $05 / 17 / 57$ & 25,600 & 120,000 \\
\hline & & & & $\mathrm{HR}$ & $10 / 08 / 70$ & 80,000 & \\
\hline 306 & 07330500 & Caddo Creek near Ardmore, Okla. & CONT & $\mathrm{N}$ & $03 / 15 / 45$ & $22,300^{\mathrm{a}}$ & 154,000 \\
\hline \multirow[t]{3}{*}{307} & 07331000 & Washita River near Dickson, Okla. & CONT & HN & 05/00/08 & 71,000 & 398,000 \\
\hline & & & & $\mathrm{N}$ & $05 / 11 / 43$ & 91,300 & \\
\hline & & & & $\mathrm{R}$ & 05/03/90 & $118,000^{\mathrm{h}}$ & \\
\hline & 07331410 & Buzzard Creek near Reagan, Okla. & CSG & $\mathrm{N}$ & $05 / 13 / 68$ & 1,040 & 23,100 \\
\hline \multirow{3}{*}{309} & 07331600 & Red River at Denison Dam near Denison, Tex. ${ }^{20}$ & CONT & $\mathrm{N}$ & $05 / 21 / 35$ & 201,000 & 608,000 \\
\hline & & & & $\mathrm{R}$ & 06/05//57 & 102,000 & \\
\hline & & & & $\mathrm{HR}$ & 05/06/90 & $145,000^{\mathrm{i}}$ & \\
\hline 310 & 07332070 & Rock Creek near Achille, Okla. & CSG & $\mathrm{N}$ & $04 / 21 / 67$ & 1,090 & 4,610 \\
\hline 311 & 07332400 & Blue River at Milburn, Okla. & CONT & $\mathrm{N}$ & $10 / 08 / 70$ & 35,100 & 137,000 \\
\hline 312 & 07332500 & Blue Creek near Blue, Okla. & CONT & $\mathrm{N}$ & $10 / 14 / 81$ & $65,200^{\mathrm{k}}$ & 177,000 \\
\hline 313 & 07332600 & Bois D’ Arc Creek near Randolph, Tex & CONT & $\mathrm{N}$ & $05 / 13 / 82$ & 19,200 & 131,000 \\
\hline
\end{tabular}

\begin{tabular}{|c|c|c|c|c|c|c|c|c|c|c|c|c|}
\hline \multirow{2}{*}{$\begin{array}{c}\text { Site } \\
\text { number } \\
\text { (fig. 1) }\end{array}$} & \multirow{2}{*}{$\begin{array}{c}\text { Contributing } \\
\text { drainage area } \\
\left(\mathrm{mi}^{2}\right)\end{array}$} & \multirow{2}{*}{ Latitude } & \multirow{2}{*}{ Longitude } & \multirow{2}{*}{$\begin{array}{c}\text { Hydrologic } \\
\text { region (E/ } \\
\text { W) }\end{array}$} & \multirow{2}{*}{$\begin{array}{c}\text { Type } \\
\text { basin (N/ } \\
\text { I/R/ } \\
\text { U) }\end{array}$} & \multicolumn{7}{|c|}{$\begin{array}{l}\text { LPIII flood frequency estimates } \\
\text { Peak discharge for indicated recurrence interval }\left(\mathrm{ft}^{3} / \mathrm{s}\right)\end{array}$} \\
\hline & & & & & & $2 \mathrm{yr}$ & $5 \mathrm{yr}$ & $10 \mathrm{yr}$ & $25 \mathrm{yr}$ & $50 \mathrm{yr}$ & $100 \mathrm{yr}$ & $500 \mathrm{yr}$ \\
\hline 285 & & & & & $R$ & 4,640 & 10,400 & 17,300 & 31,800 & 49,000 & 72,200 & 186,000 \\
\hline \multirow[t]{2}{*}{286} & 208 & 0351030 & 0981520 & w & $\mathrm{N}$ & 1,260 & 2,930 & 4,690 & 7,910 & 11,200 & 15,400 & 30,200 \\
\hline & & & & & $\mathrm{R}$ & 1,480 & 3,520 & 5,730 & 9,940 & 14,400 & 20,300 & 42,100 \\
\hline 287 & 40.1 & 0350325 & 0981041 & E & $\mathrm{R}$ & 359 & 791 & 1,140 & 1,640 & 2,030 & 2,440 & 3,430 \\
\hline 288 & 23.8 & 0350844 & 0975703 & E & $\mathrm{N}$ & 719 & 1,640 & 2,580 & 4,240 & 5,900 & 7,980 & 15,000 \\
\hline 289 & 52.0 & 0350318 & 0975711 & E & $\mathrm{N}$ & 437 & 998 & 1,550 & 2,510 & 3,440 & 4,580 & 8,260 \\
\hline 290 & 59.4 & 0350300 & 0975100 & E & $\mathrm{N}$ & 1,670 & 3,110 & 4,330 & 6,170 & 7,760 & 9,560 & 14,600 \\
\hline 291 & 0.30 & 0350441 & 0974710 & E & $\mathrm{N}$ & 30 & 48 & 63 & 84 & 103 & 124 & 184 \\
\hline 292 & 35.2 & 0350238 & 0974928 & E & $\mathrm{N}$ & 1,740 & 3,020 & 4,050 & 5,590 & 6,910 & 8,380 & 12,500 \\
\hline \multirow[t]{2}{*}{293} & 208 & 0345641 & 0975708 & E & $\mathrm{N}$ & 3,320 & 7,510 & 11,900 & 20,200 & 28,700 & 39,900 & 80,300 \\
\hline & & & & & $\mathrm{R}$ & 2,900 & 4,750 & 6,200 & 8,310 & 10,100 & 12,000 & 17,300 \\
\hline 294 & 4,706 & 0345818 & 0975221 & E & $\mathrm{N}$ & 11,800 & 22,900 & 32,500 & 47,700 & 61,300 & 77,000 & 123,000 \\
\hline 295 & 7.57 & 0345644 & 0975018 & E & $\mathrm{R}$ & 337 & 794 & 1,310 & 2,340 & 3,470 & 5,030 & 11,200 \\
\hline 296 & 0.88 & 0345706 & 0975048 & E & $\mathrm{R}$ & 153 & 216 & 253 & 294 & 321 & 346 & 396 \\
\hline 297 & 33.0 & 0345935 & 0974540 & E & $\mathrm{R}$ & 1,210 & 2,970 & 4,580 & 7,060 & 9,200 & 11,600 & 17,800 \\
\hline 298 & 4,787 & 0345533 & 0974625 & E & $\mathrm{R}$ & 7,710 & 12,900 & 16,700 & 21,600 & 25,400 & 29,200 & 38,400 \\
\hline \multirow[t]{2}{*}{299} & 5,330 & 0344517 & 0971504 & E & $\mathrm{N}$ & 14,000 & 21,400 & 26,200 & 31,900 & 35,900 & 39,700 & 47,900 \\
\hline & & & & & $\mathrm{R}$ & 11,400 & 18,800 & 24,400 & 32,200 & 38,400 & 45,000 & 61,800 \\
\hline \multirow[t]{2}{*}{300} & 145 & 0344146 & 0973555 & E & $\mathrm{N}$ & 10,000 & 16,100 & 21,100 & 28,500 & 35,000 & 42,400 & 63,500 \\
\hline & & & & & $\mathrm{R}$ & 3,570 & 6,980 & 10,000 & 14,800 & 19,200 & 24,300 & 39,600 \\
\hline \multirow[t]{2}{*}{301} & 206 & 0344436 & 0972418 & E & $\mathrm{N}$ & 9,260 & 17,700 & 25,500 & 38,400 & 50,800 & 65,800 & 114,000 \\
\hline & & & & & $\mathrm{R}$ & 5,510 & 9,800 & 13,200 & 18,100 & 22,100 & 26,500 & 38,100 \\
\hline 302 & 604 & 0343239 & 0971449 & E & $\mathrm{R}$ & 11,600 & 19,800 & 25,800 & 33,900 & 40,200 & 46,700 & 62,600 \\
\hline 303 & 18.7 & 0342650 & 0970740 & E & $\mathrm{N}$ & 1,990 & 4,520 & 7,120 & 11,800 & 16,500 & 22,600 & 43,300 \\
\hline 304 & 44.1 & 0342943 & 0965918 & E & $\mathrm{R}$ & 5,100 & 7,100 & 8,540 & 10,500 & 12,100 & 13,700 & 18,000 \\
\hline 305 & 138 & 0342350 & 0970210 & E & $\mathrm{N}$ & 4,510 & 10,500 & 16,900 & 28,900 & 41,500 & 58,100 & 118,000 \\
\hline 306 & 298 & 0341433 & 0970628 & E & $\mathrm{N}$ & 8,150 & 15,500 & 21,900 & 32,100 & 41,200 & 51,900 & 83,400 \\
\hline \multirow[t]{2}{*}{307} & 7,202 & 0341400 & 0965832 & E & $\mathrm{N}$ & 22,700 & 41,200 & 56,100 & 78,000 & 96,400 & 117,000 & 172,000 \\
\hline & & & & & $\mathrm{R}$ & 29,500 & 46,700 & 59,100 & 75,900 & 89,000 & 103,000 & 137,000 \\
\hline 308 & 4.30 & 0341950 & 0963928 & E & $\mathrm{N}$ & 465 & 711 & 898 & 1,160 & 1,380 & 1,620 & 2,260 \\
\hline \multirow[t]{2}{*}{309} & 33,784 & 0334908 & 0963347 & $\mathrm{E}$ & $\mathrm{N}$ & 87,000 & 140,000 & 177,000 & 226,000 & 262,000 & 299,000 & 386,000 \\
\hline & & & & & $\mathrm{R}$ & 30,400 & 52,700 & 68,900 & 90,600 & 107,000 & 124,000 & 165,000 \\
\hline 310 & 0.72 & 0334835 & 0962238 & E & $\mathrm{N}$ & 396 & 683 & 921 & 1,280 & 1,590 & 1,940 & 2,950 \\
\hline 311 & 203 & 0341502 & 0963255 & E & $\mathrm{N}$ & 8,630 & 17,200 & 24,600 & 35,900 & 45,800 & 56,800 & 87,600 \\
\hline 312 & 476 & 0335949 & 0961427 & E & $\mathrm{N}$ & 9,400 & 17,500 & 24,800 & 36,600 & 47,600 & 60,600 & 101,000 \\
\hline 313 & 178 & 0332832 & 0961252 & $\mathrm{E}$ & $\mathrm{N}$ & 8,220 & 12,600 & 15,500 & 19,100 & 21,800 & 24,400 & 30,200 \\
\hline
\end{tabular}




\begin{tabular}{|c|c|c|c|c|c|c|c|}
\hline \multirow[b]{2}{*}{$\begin{array}{c}\text { Site } \\
\text { number } \\
\text { (fig. 1) }\end{array}$} & \multirow[b]{2}{*}{$\begin{array}{l}\text { Station } \\
\text { number }\end{array}$} & \multirow[b]{2}{*}{ Station name } & \multirow[b]{2}{*}{$\begin{array}{l}\text { Type of } \\
\text { station } \\
\text { (CNNT/ } \\
\text { CSG) }\end{array}$} & \multicolumn{3}{|c|}{ Documented extreme peak discharge } & \multirow[b]{2}{*}{$\begin{array}{c}\text { Potential } \\
\text { extreme } \\
\text { peak } \\
\text { discharge } \\
\left(\mathbf{f t}^{3} / \mathrm{s}\right) \\
\text { (table 4) }\end{array}$} \\
\hline & & & & $\begin{array}{c}\text { Type of } \\
\text { record (H/ } \\
\text { I/N/ } \\
\text { R/U) }\end{array}$ & Date & $\begin{array}{c}\text { Discharge } \\
\left(\mathrm{ft}^{\mathbf{3}} / \mathrm{s}\right)\end{array}$ & \\
\hline 314 & 07333330 & Chickasaw Creek Tributary near Stringtown, Okla. & CSG & $\begin{array}{l}\mathrm{N} \\
\mathrm{HN}\end{array}$ & $\begin{array}{l}04 / 20 / 71 \\
10 / 0 / 81\end{array}$ & $\begin{array}{l}4,930 \\
6,320^{\mathrm{k}}\end{array}$ & 18,200 \\
\hline 315 & 07333500 & Chickasaw Creek near Stringtown, Okla. ${ }^{21}$ & CSG & $\mathrm{N}$ & $10 / 14 / 62$ & $18,800^{\mathrm{b}}$ & 67,700 \\
\hline 316 & 07333800 & McGee Creek near Stringtown, Okla. ${ }^{21}$ & CSG & $\mathrm{N}$ & $11 / 24 / 73$ & 11,100 & 101,000 \\
\hline 317 & 07334000 & Muddy Boggy Creek near Farris, Okla. & CONT & $\begin{array}{l}\mathrm{N} \\
\mathrm{R}\end{array}$ & $\begin{array}{l}06 / 17 / 45 \\
05 / 05 / 90\end{array}$ & $\begin{array}{l}61,900^{\mathrm{a}} \\
49,800^{\mathrm{h}}\end{array}$ & 227,000 \\
\hline 318 & 07335000 & Clear Boggy Creek near Caney, Okla. & CONT & $\begin{array}{l}\mathrm{HN} \\
\mathrm{N} \\
\mathrm{R}\end{array}$ & $\begin{array}{l}02 / 00 / 38 \\
12 / 11 / 46 \\
10 / 1481\end{array}$ & $\begin{array}{l}54,600^{\mathrm{a}} \\
52,800 \\
53500^{\mathrm{k}}\end{array}$ & 202,000 \\
\hline 319 & 07335300 & Muddy Boggy Creek near Unger, Okla. & CONT & $\mathrm{N}$ & 05/10//90 & $76,700^{\mathrm{h}}$ & 282,000 \\
\hline 320 & 07335310 & Rock Creek near Boswell, Okla. & CSG & $\mathrm{N}$ & $05 / 13 / 68$ & 550 & 5,940 \\
\hline 321 & 07335320 & Bokchito Creek near Soper, Okla. & CSG & $\mathrm{N}$ & $10 / 30 / 74$ & 5,840 & 50,100 \\
\hline 322 & 07335500 & Red River at Arthur City, Tex. & CONT & $\begin{array}{l}\mathrm{N} \\
\mathrm{R}\end{array}$ & $\begin{array}{c}05 / 28 / 08 \\
05 / 04 / 90\end{array}$ & $\begin{array}{l}400,000 \\
275,000 \mathrm{~h}^{\mathrm{h}}\end{array}$ & 630,000 \\
\hline 323 & 07335700 & Kiamichi River near Big Cedar, Okla. ${ }^{22}$ & CONT & $\mathrm{N}$ & $05 / 19 / 90$ & 27,400 & 74,200 \\
\hline 324 & 07335760 & Kiamichi River Tributary near Albion, Okla. & CSG & $\mathrm{N}$ & 12/09/71 & 1,070 & 8,810 \\
\hline 325 & 07335790 & Kiamichi River near Clayton, Okla. & CONT & $\mathrm{R}$ & 05/04/90 & $40,200^{\mathrm{h}}$ & 201,000 \\
\hline 326 & 07336000 & Tenmile Creek near Miller, Okla. $^{1}$ & CSG & $\mathrm{N}$ & 03/28/77 & 10,600 & 92,400 \\
\hline 327 & 07336200 & Kiamichi River near Antlers, Okla. ${ }^{22}$ & CONT & $\begin{array}{l}\mathrm{N} \\
\mathrm{R}\end{array}$ & $\begin{array}{l}03 / 28 / 77 \\
05 / 03 / 90\end{array}$ & $\begin{array}{l}50,000 \\
62,300^{\mathrm{h}}\end{array}$ & 230,000 \\
\hline 328 & 07336500 & Kiamichi River near Belzoni, Okla. & CONT & $\begin{array}{l}\mathrm{HN} \\
\mathrm{N}\end{array}$ & $\begin{array}{l}10 / 00 / 15 \\
02 / 18 / 38\end{array}$ & $\begin{array}{l}72,000^{\mathrm{a}} \\
71,400\end{array}$ & 246,000 \\
\hline 329 & 07336520 & Frazier Creek near Oleta, Okla. & CSG & $\mathrm{N}$ & $11 / 01 / 84$ & 7,400 & 54,000 \\
\hline 330 & 07336710 & Rock Creek near Sawyer, Okla. & CSG & $\mathrm{N}$ & 12/09/71 & 1,560 & 19,100 \\
\hline 331 & 07336750 & Little Pine Creek near Kanawha, Tex. & CONT & $\mathrm{N}$ & $12 / 10 / 71$ & 30,200 & 96,400 \\
\hline 332 & 07336780 & Perry Creek near Idabel, Okla. & CSG & $\mathrm{N}$ & 04/22/64 & 4,400 & 33,500 \\
\hline 333 & 07336785 & Bokchito Creek near Garvin, Okla. & CSG & $\mathrm{N}$ & 12/09/71 & 1,270 & 17,100 \\
\hline 334 & 07336800 & Pecan Bayou near Clarksville, Tex. ${ }^{23}$ & CONT & $\mathrm{N}$ & $12 / 10 / 71$ & 21,300 & 107,000 \\
\hline 335 & 07336820 & Red River near DeKalb, Tex. & CONT & $\begin{array}{c}\mathrm{HR} \\
\mathrm{R}\end{array}$ & $\begin{array}{l}06 / 107 / 57 \\
05 / 06 / 90\end{array}$ & $\begin{array}{l}205,000 \\
279,000^{\mathrm{h}}\end{array}$ & 642,000 \\
\hline 336 & 07337220 & Big Branch near Ringold, Okla. & CSG & $\mathrm{N}$ & $10 / 31 / 72$ & 1,540 & 12,000 \\
\hline 337 & 07337500 & Little River near Wright City, Okla. & CONT & $\begin{array}{l}\mathrm{N} \\
\mathrm{R}\end{array}$ & $\begin{array}{l}05 / 06 / 61 \\
12 / 1 / 1 / 71\end{array}$ & $\begin{array}{l}78,200 \\
11,200^{\mathrm{m}}\end{array}$ & 195,000 \\
\hline 338 & 07337900 & Glover River near Glover, Okla. & CONT & $\begin{array}{l}\mathrm{HN} \\
\mathrm{N}\end{array}$ & $\begin{array}{l}05 / 00 / 61 \\
12 / 10 / 71\end{array}$ & $\begin{array}{l}88,200 \\
98,600^{\mathrm{m}}\end{array}$ & 156,000 \\
\hline 339 & 07338500 & Little River below Lukfata Creek near Idabel, Okla. ${ }^{24}$ & CONT & $\begin{array}{l}\mathrm{HN} \\
\mathrm{N} \\
\mathrm{R}\end{array}$ & $\begin{array}{l}02 / 00 / 38 \\
09 / 17 / 50 \\
12 / 1 / 1 / 71\end{array}$ & $\begin{array}{c}86,000 \\
66,100 \\
103,000^{\mathrm{m}}\end{array}$ & 235,000 \\
\hline 340 & 07338520 & Yanubbee Creek near Broken Bow, Okla. & CSG & $\mathrm{N}$ & $05 / 03 / 84$ & 4,700 & 37,200 \\
\hline 341 & 07338700 & Two Mile Creek near Hatfield, Ark. & CSG & $\mathrm{N}$ & 05/13/68 & 6,260 & 49,100 \\
\hline 342 & 07338750 & Mountain Fork at Smithville, Okla. & CONT & $\mathrm{N}$ & 10/06/98 & 46,500 & 157,000 \\
\hline 343 & 07338780 & Mountain Fork Tributary near Smithville, Okla. & CSG & $\mathrm{N}$ & $05 / 13 / 82$ & 1,030 & 5,390 \\
\hline 344 & 07339000 & Mountain Fork near Eagletown, Okla. & CONT & $\begin{array}{l}\mathrm{HN} \\
\mathrm{N} \\
\mathrm{R}\end{array}$ & $\begin{array}{l}08 / 18 / 15 \\
05 / 20 / 60 \\
06 / 02 / 90\end{array}$ & $\begin{array}{c}92,000^{\mathrm{a}} \\
101,000^{\mathrm{b}} \\
18,200^{2}\end{array}$ & 207,000 \\
\hline
\end{tabular}

\begin{tabular}{|c|c|c|c|c|c|c|c|c|c|c|c|c|}
\hline \multirow{2}{*}{$\begin{array}{c}\text { Site } \\
\text { number } \\
\text { (fig. 1) }\end{array}$} & \multirow{2}{*}{$\begin{array}{c}\text { Contributing } \\
\text { drainage area } \\
\left(\mathrm{mi}^{2}\right)\end{array}$} & \multirow{2}{*}{ Latitude } & \multirow{2}{*}{ Longitude } & \multirow{2}{*}{$\begin{array}{l}\text { Hydrologic } \\
\text { region (E/ } \\
\text { W) }\end{array}$} & \multirow{2}{*}{$\begin{array}{c}\text { Type } \\
\text { basin (N/ } \\
\text { //R/ } \\
\text { U) }\end{array}$} & \multicolumn{7}{|c|}{$\begin{array}{l}\text { LPIII flood frequency estimates } \\
\text { Peak discharge for indicated recurrence interval }\left(\mathrm{ft}^{3} / \mathrm{s}\right)\end{array}$} \\
\hline & & & & & & $2 \mathrm{yr}$ & $5 \mathrm{yr}$ & $10 \mathrm{yr}$ & $25 \mathrm{yr}$ & $50 \mathrm{yr}$ & $100 \mathrm{yr}$ & $500 \mathrm{yr}$ \\
\hline 314 & 3.19 & 0342933 & 0955639 & E & $\mathrm{N}$ & 1,610 & 3,400 & 5,110 & 7,970 & 10,700 & 14,000 & 24,500 \\
\hline 315 & 32.7 & 0342741 & 0960136 & E & $\mathrm{N}$ & 7,580 & 10,800 & 13,100 & 16,200 & 18,600 & 21,200 & 27,500 \\
\hline 316 & 86.6 & 0342633 & 0955210 & E & $\mathrm{N}$ & 6,660 & 8,870 & 10,300 & 12,000 & 13,300 & 14,500 & 17,300 \\
\hline \multirow{2}{*}{317} & 1,087 & 0341617 & 0955443 & E & $\mathrm{N}$ & 19,500 & 29,400 & 36,700 & 47,000 & 55,300 & 64,200 & 87,700 \\
\hline & & & & & $\mathrm{R}$ & 14,700 & 21,500 & 28,300 & 40,300 & 52,400 & 68,000 & 124,000 \\
\hline \multirow[t]{2}{*}{318} & 720 & 0341509 & 0961219 & E & $\mathrm{N}$ & 14,000 & 28,600 & 42,200 & 64,600 & 85,500 & 111,000 & 188,000 \\
\hline & & & & & $\mathrm{R}$ & 11,000 & 18,200 & 23,800 & 31,400 & 38,700 & 46,000 & 65,800 \\
\hline 319 & 2,273 & 0340136 & 0954500 & E & $\mathrm{N}$ & 20,900 & 32,900 & 42,100 & 54,900 & 65,500 & 76,800 & 107,000 \\
\hline 320 & 0.94 & 0335757 & 0955202 & E & $\mathrm{N}$ & 250 & 428 & 562 & 749 & 899 & 1,060 & 1,460 \\
\hline 321 & 16.6 & 0340220 & 0954010 & E & $\mathrm{N}$ & 3,230 & 5,100 & 6,440 & 8,240 & 9,630 & 11,100 & 14,600 \\
\hline \multirow[t]{2}{*}{322} & 38,595 & 0335230 & 0953006 & E & $\mathrm{N}$ & 80,600 & 146,000 & 202,000 & 287,000 & 361,000 & 446,000 & 688,000 \\
\hline & & & & & $\mathrm{R}$ & 57,300 & 86,500 & 111,000 & 150,000 & 185,000 & 226,000 & 349,000 \\
\hline 323 & 40.1 & 0343818 & 0943645 & E & $\mathrm{N}$ & 9,330 & 14,700 & 18,400 & 23,200 & 26,900 & 30,600 & 39,500 \\
\hline 324 & 1.43 & 0343740 & 0950250 & E & $\mathrm{N}$ & 222 & 538 & 862 & 1,430 & 2,000 & 2,700 & 5,000 \\
\hline 325 & 708 & 0343432 & 0952026 & E & $\mathrm{R}$ & 17,000 & 22,700 & 26,800 & 32,200 & 36,500 & 41,000 & 52,500 \\
\hline 326 & 68.0 & 0341755 & 0954440 & E & $\mathrm{N}$ & 3,620 & 5,080 & 6,130 & 7,560 & 8,690 & 9,890 & 13,000 \\
\hline \multirow[t]{2}{*}{327} & 1,138 & 0341455 & 0953618 & E & $\mathrm{N}$ & 28,200 & 37,400 & 42,700 & 48,800 & 53,000 & 56,900 & 65,000 \\
\hline & & & & & $\mathrm{R}$ & 27,500 & 37,700 & 44,700 & 53,900 & 61,000 & 68,200 & 86,100 \\
\hline 328 & 1,423 & 0341202 & 0952903 & E & $\mathrm{N}$ & 34,500 & 49,400 & 59,400 & 72,000 & 81,400 & 90,800 & 113,000 \\
\hline 329 & 19.4 & 0341150 & 0952100 & E & $\mathrm{N}$ & 2,500 & 4,570 & 6,220 & 8,570 & 10,500 & 12,600 & 18,000 \\
\hline 330 & 3.39 & 0340150 & 0952130 & E & $\mathrm{N}$ & 790 & 1,170 & 1,440 & 1,790 & 2,060 & 2,350 & 3,050 \\
\hline 331 & 75.4 & 0335026 & 0951555 & E & $\mathrm{N}$ & 5,890 & 10,100 & 13,500 & 18,600 & 23,000 & 28,100 & 42,300 \\
\hline 332 & 7.53 & 0335344 & 0945315 & E & $\mathrm{N}$ & 2,220 & 3,020 & 3,580 & 4,310 & 4,870 & 5,440 & 6,860 \\
\hline 333 & 2.96 & 0335344 & 0945423 & E & $\mathrm{N}$ & 726 & 1,020 & 1,210 & 1,450 & 1,630 & 1,810 & 2,220 \\
\hline 334 & 100 & 0334107 & 0945941 & E & $\mathrm{N}$ & 4,280 & 7,210 & 9,560 & 13,000 & 15,900 & 19,200 & 28,100 \\
\hline 335 & 41,412 & 0334059 & 0944139 & E & $\mathrm{R}$ & 72,300 & 110,000 & 140,000 & 183,000 & 220,000 & 261,000 & 374,000 \\
\hline 336 & 1.99 & 0341027 & 0950433 & E & $\mathrm{N}$ & 450 & 857 & 1,200 & 1,720 & 2,160 & 2,660 & 4,050 \\
\hline \multirow[t]{2}{*}{337} & 645 & 0340410 & 0950247 & E & $\mathrm{N}$ & 30,500 & 49,700 & 64,100 & 83,800 & 99,500 & 116,000 & 158,000 \\
\hline & & & & & $\mathrm{R}$ & 6,460 & 7,840 & 8,740 & 9,870 & 10,700 & 11,600 & 13,600 \\
\hline 338 & 315 & 0340551 & 0945407 & E & $\mathrm{N}$ & 28,000 & 44,300 & 56,400 & 73,100 & 86,600 & 101,000 & 138,000 \\
\hline \multirow[t]{2}{*}{339} & 1,226 & 0335628 & 0944530 & E & $\mathrm{N}$ & 27,500 & 46,100 & 60,100 & 79,500 & 95,200 & 112,000 & 155,000 \\
\hline & & & & & $\mathrm{R}$ & 12,300 & 19,300 & 27,800 & 45,600 & 66,900 & 98,500 & 244,000 \\
\hline 340 & 9.10 & 0340345 & 0944422 & E & $\mathrm{N}$ & 1,780 & 3,110 & 4,110 & 5,500 & 6,600 & 7,750 & 10,600 \\
\hline 341 & 15.9 & 0343052 & 0942014 & $\mathrm{E}$ & $\mathrm{N}$ & 1,960 & 3,530 & 4,870 & 6,950 & 8,810 & 11,000 & 17,300 \\
\hline 342 & 320 & 0342744 & 0943806 & E & $\mathrm{N}$ & 26,300 & 35,800 & 42,100 & 50,300 & 56,400 & 62,500 & 77,300 \\
\hline 343 & 0.85 & 0342948 & 0944006 & E & $\mathrm{N}$ & 199 & 356 & 488 & 689 & 865 & 1,070 & 1,640 \\
\hline \multirow[t]{2}{*}{344} & 787 & 0340230 & 0943711 & E & $\mathrm{N}$ & 39,400 & 64,400 & 82,300 & 106,000 & 124,000 & 143,000 & 187,000 \\
\hline & & & & & $\mathrm{R}$ & 9,260 & 11,800 & 13,800 & 16,700 & 19,100 & 21,700 & 28,800 \\
\hline
\end{tabular}


Table 1. Documented and potential extreme peak discharges and flood frequency estimates for selected streamflow-gaging stations with at least

\begin{tabular}{|c|c|c|c|c|c|c|c|}
\hline \multirow[b]{2}{*}{$\begin{array}{c}\text { Site } \\
\text { number } \\
\text { (fig. 1) }\end{array}$} & \multirow[b]{2}{*}{$\begin{array}{l}\text { Station } \\
\text { number }\end{array}$} & \multirow[b]{2}{*}{ Station name } & \multirow[b]{2}{*}{$\begin{array}{l}\text { Type of } \\
\text { station } \\
\text { (CONT/ } \\
\text { CSG) }\end{array}$} & \multicolumn{3}{|c|}{ Documented extreme peak discharge } & \multirow[b]{2}{*}{$\begin{array}{c}\text { Potential } \\
\text { extreme } \\
\text { peak } \\
\text { discharge } \\
\left(\mathrm{ft}^{3} / \mathrm{s}\right) \\
\text { (table 4) }\end{array}$} \\
\hline & & & & $\begin{array}{c}\text { Type of } \\
\text { record (H/ } \\
\text { //N/ } \\
\text { R/U) }\end{array}$ & Date & $\begin{array}{c}\text { Discharge } \\
\left(\mathrm{ft}^{3} / \mathbf{s}\right)\end{array}$ & \\
\hline \multirow[t]{3}{*}{345} & 07339500 & Rolling Fork near DeQueen, Ark. ${ }^{25}$ & CSG & $\mathrm{HN}$ & $08 / 27 / 47$ & $110,000^{a, b}$ & 132,000 \\
\hline & & & & $\mathrm{N}$ & $12 / 10 / 71$ & 71,000 & \\
\hline & & & & $\mathrm{R}$ & $05 / 17 / 82$ & 6,320 & \\
\hline 346 & 07339800 & Pepper Creek near DeQueen, Ark. & CSG & $\mathrm{N}$ & $05 / 13 / 68$ & 6,240 & 30,300 \\
\hline \multirow{3}{*}{347} & 07340000 & Little River near Horatio. Ark. & CONT & $\mathrm{HN}$ & $08 / 00 / 15$ & $124,000^{\mathrm{a}}$ & 296,000 \\
\hline & & & & $\mathrm{N}$ & $03 / 30 / 45$ & 120,000 & \\
\hline & & & & $\mathrm{R}$ & $12 / 10 / 71$ & 65,100 & \\
\hline 348 & 07340200 & West Flat Creek near Foremean, Ark. & CSG & $\mathrm{N}$ & $12 / 02 / 82$ & 3,800 & 40,700 \\
\hline \multirow{2}{*}{349} & 07340300 & Cossalot River near Vandervoort, Ark. & CONT & $\mathrm{HN}$ & $05 / 06 / 61$ & 48,000 & 102,000 \\
\hline & & & & $\mathrm{N}$ & $12 / 02 / 82$ & 32,000 & \\
\hline \multirow[t]{2}{*}{350} & 07340500 & Cossalot River near DeQueen, Ark. 25 & CSG & $\mathrm{N}$ & $05 / 13 / 68$ & 122,000 & 162,000 \\
\hline & & & & $\mathrm{R}$ & $07 / 02 / 83$ & 22,600 & \\
\hline 351 & 07340530 & Mill Slough Tributary near Lockesburg, Ark. & CSG & $\mathrm{N}$ & $12 / 26 / 82$ & 719 & 4,090 \\
\hline 352 & 07343300 & Cuthand Creek near Bogata, Tex. & CONT & $\mathrm{N}$ & $12 / 10 / 71$ & 20,400 & 93,000 \\
\hline
\end{tabular}

\section{${ }^{a}$ Patterson (1964)}

Crippen and Bue (1977)

Walters and Tortorelli (1998)

Bingham, Bergman and Thomas (1974)

U.S. Geological Survey (1954)

${ }^{\mathrm{g}}$ Hauth (1985)

${ }^{\mathrm{h}}$ Tortorelli (1996b)

U.S. Army Corps of Engineers, Tulsa District (1990)

Corley and Huntzinger (1979)

Buckner and Kurklin (1984)

Burnham (1939)

Thomas and Corley (1973)

Continuous-record gage prior to WY 1971

Frequency analysis includes streamflow record from nearby station 07161000

${ }^{4}$ Historical record length assumed equal to that for nearby station 07188000

${ }^{5}$ Continuous-record gage prior to WY 1960
${ }^{6}$ Continuous-record gage prior to WY 1973

Historical record length assumed to start from same year as that for nearby station 07196500 for unregulated streamflow period

${ }_{9}^{8}$ Historical record length assumed equal to that for nearby station 07230500 for unregulated streamflow period

Pre- and post-irrigation development as defined in Wahl and Tortorelli (1997)
10 Historical record length

${ }^{11}$ Historical record length assumed equal to that for nearby station 07241000

${ }^{12}$ Was 07250000, Lee Creek near Van Buren, Ark., prior to WY 1993

${ }^{13}$ Was 07250500, Arkansas River at Van Buren, Ark., prior to WY 1970

${ }_{15}^{14}$ Historical record length assumed to start from same year as that for nearby station 07299850 for unregulated streamflow period

${ }_{16}^{15}$ Frequency analysis includes streamflow record from nearby station 07302000

${ }_{17}^{16}$ Historical record length assumed equal to that for nearby station 07313500

Streamflow data conpud fiom ninhow to nloodwater retarding structure

${ }_{19}$ Frequency analysis includes streamflow record from nearby station 07327500 , not shown in table
8 years of annual peak-discharge data from unregulated, regulated, and urban basins within and near Oklahoma-Continued

\begin{tabular}{|c|c|c|c|c|c|c|c|c|c|c|c|c|}
\hline \multirow{2}{*}{$\begin{array}{c}\text { Site } \\
\text { number } \\
\text { (fig. 1) }\end{array}$} & \multirow{2}{*}{$\begin{array}{c}\text { Contributing } \\
\text { drainage area } \\
\left(\mathbf{m i}^{2}\right)\end{array}$} & \multirow{2}{*}{ Latitude } & \multirow{2}{*}{ Longitude } & \multirow{2}{*}{$\begin{array}{l}\text { Hydrologic } \\
\text { region (E/ } \\
\text { W) }\end{array}$} & \multirow{2}{*}{$\begin{array}{c}\text { Type } \\
\text { basin (N/ } \\
\text { I/R/ } \\
\text { U) }\end{array}$} & \multicolumn{7}{|c|}{$\begin{array}{l}\text { LPIII flood frequency estimates } \\
\text { Peak discharge for indicated recurrence interval }\left(\mathrm{ft}^{3} / \mathrm{s}\right)\end{array}$} \\
\hline & & & & & & $2 \mathrm{yr}$ & $5 \mathrm{yr}$ & $10 \mathrm{yr}$ & $25 \mathrm{yr}$ & $50 \mathrm{yr}$ & $100 \mathrm{yr}$ & $500 \mathrm{yr}$ \\
\hline \multirow[t]{2}{*}{345} & 182 & 0340251 & 0942447 & E & $\mathrm{N}$ & 15,700 & 31,200 & 45,300 & 68,100 & 89,100 & 114,000 & 190,000 \\
\hline & & & & & $\mathrm{R}$ & 2,300 & 3,370 & 4,290 & 5,750 & 7,070 & 8,640 & 13,500 \\
\hline 346 & 6.41 & 0340244 & 0941813 & E & $\mathrm{N}$ & 961 & 2,400 & 3,840 & 6,330 & 8,720 & 11,600 & 20,600 \\
\hline \multirow{2}{*}{347} & 2,662 & 0335510 & 0942315 & E & $\mathrm{N}$ & 46,400 & 71,300 & 89,200 & 113,000 & 132,000 & 152,000 & 201,000 \\
\hline & & & & & $\mathrm{R}$ & 25,800 & 35,300 & 41,500 & 49,100 & 54,700 & 60,200 & 72,900 \\
\hline 348 & 10.7 & 0334513 & 0942328 & E & $\mathrm{N}$ & 1,540 & 2,650 & 3,490 & 4,660 & 5,590 & 6,580 & 9,100 \\
\hline 349 & 89.6 & 0342246 & 0941408 & E & $\mathrm{N}$ & 14,600 & 25,400 & 33,800 & 45,600 & 55,400 & 65,800 & 93,000 \\
\hline \multirow[t]{2}{*}{350} & 360 & 0340245 & 0941242 & E & $\mathrm{N}$ & 27,800 & 46,600 & 61,800 & 84,300 & 104,000 & 125,000 & 185,000 \\
\hline & & & & & $\mathrm{R}$ & 8,080 & 13,200 & 16,800 & 21,400 & 24,900 & 28,400 & 36,500 \\
\hline 351 & 0.64 & 0335804 & 0941125 & $\mathrm{E}$ & $\mathrm{N}$ & 189 & 337 & 460 & 643 & 801 & 977 & 1,470 \\
\hline & 69.0 & 0333251 & 0951022 & E & $\mathrm{N}$ & 4,740 & 7,580 & 9,800 & 13,000 & 15,700 & 18,700 & 26,700 \\
\hline
\end{tabular}

${ }^{21}$ Continuous-record gage prior to WY 1969

${ }_{23}^{2}$ Historical record length assumed to start from same year as that for nearby station 07336500 for unregulated streamflow period ${ }^{23}$ Historical record length assumed to start from same year as that for nearby station 07335500 for regulated streamflow period

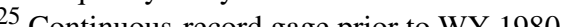


Table 2. Documented and potential extreme peak discharges for selected indirect measurement sites without streamflow-gaging stations and

[N, unregulated; R, regulated; U, urban; ft $\mathrm{t}^{3} / \mathrm{s}$, cubic feet per second; mi2', square miles; E, sites east of 98 degrees 15 minutes longitude;

\begin{tabular}{|c|c|c|}
\hline $\begin{array}{c}\text { Site } \\
\text { number } \\
\text { (fig. 2) }\end{array}$ & $\begin{array}{l}\text { Station } \\
\text { number }\end{array}$ & Station name or indirect measurement site name \\
\hline 353 & & Anderson Creek near Freedom, Okla. \\
\hline 354 & 07246500 & Arkansas River near Sallisaw, Okla. \\
\hline 355 & 07324500 & Barnitz Creek near Arapaho, Okla. \\
\hline 356 & & Barnitz Creek Tributary near Arapaho, Okla. ${ }^{1}$ \\
\hline 357 & & Beaver Creek Tributary near Arapaho, Okla. \\
\hline 358 & & Bird Creek at Nelagoney, Okla. \\
\hline 359 & & Black Bear Creek near Garber, Okla. ${ }^{2}$ \\
\hline 360 & & Black Bear Creek near Skedee, Okla. \\
\hline 361 & 07247250 & Black Fork below Big Creek near Page, Okla. \\
\hline 362 & 07232010 & Blue Creek near Blocker, Okla. \\
\hline 363 & 07159500 & Bluff Creek above Lake Hefner near Oklahoma City, Okla. \\
\hline 364 & 07159450 & Bluff Creek at Oklahoma City, Okla. \\
\hline 365 & & Boggy Creek at Lahoma Road at Enid, Okla. ${ }^{2}$ \\
\hline 366 & & Boggy Ck Diversion Canal below Rupe Ave in Enid, Okla. ${ }^{2}$ \\
\hline 367 & 07249080 & Brazil Creek near Walls, Okla. \\
\hline 368 & & Brock Creek at SW 29th Street at Oklahoma City, Okla. ${ }^{3}$ \\
\hline 369 & & Brock Creek at SW 44th Street at Oklahoma City, Okla. ${ }^{3}$ \\
\hline 370 & & Brock Creek at SW 59th Street at Oklahoma City, Okla. ${ }^{3}$ \\
\hline 371 & & Brookhollow Ck below 136th East Ave Bridge at Tulsa, Okla. ${ }^{4}$ \\
\hline 372 & & Buffalo Creek near Tiff City, Mo. ${ }^{1}$ \\
\hline 373 & 07229000 & Canadian River near Newcastle, Okla. \\
\hline 374 & & Caney Creek southwest of Wauhillau, Okla. \\
\hline 375 & 07228600 & Canyon View Creek near Geary, Okla. \\
\hline 376 & & Chisholm Creek at Village Drive, The Village, Okla. \\
\hline 377 & & Coal Creek at Tulsa, Okla. \\
\hline 378 & 07332900 & Coal Creek near Lehigh, Okla. ${ }^{5}$ \\
\hline 379 & & Coal Creek of Deep Fork near Dewar, Okla. \\
\hline 380 & & Cooley Creek at 129 th East Ave at Tulsa, Okla. ${ }^{4}$ \\
\hline 381 & & Cooley Ck Trib at Interstate 44 and 129th East Ave at Tulsa, Okla. ${ }^{4}$ \\
\hline 382 & & Coon Creek near Wewoka, Okla. 1,6 \\
\hline 383 & & Cottonwood Creek near Guthrie, Okla. ${ }^{7}$ \\
\hline 384 & 07159720 & Cottonwood Creek near Navina, Okla. \\
\hline 385 & & Cow Creek near Comanche, Okla. ${ }^{1}$ \\
\hline 386 & & Crawfish Creek near Mountain View, Okla. \\
\hline
\end{tabular}

Potential

Type
basin (N/ Date $\quad \begin{gathered}\text { Discharge } \\ \left(\mathrm{ft}^{3} / \mathrm{s}\right)\end{gathered}$ $\begin{gathered}\begin{array}{c}\text { extreme } \\ \text { peak } \\ \text { discharge } \\ \left(\mathbf{H}^{3} / \mathrm{s}\right)\end{array}\end{gathered}$

$\begin{array}{llr}05 / 16 / 57 & 14,600 & 50,600\end{array}$

$105 / 12 / 50 \quad 442,000 \quad 856,000$

$05 / 27 / 57 \quad 544,000$

110,000

$05 / 16 / 51 \quad 2.990 \quad 7.780$

$\begin{array}{lll}05 / 16 / 51 & 1,590 & 3,320\end{array}$

$05 / 19 / 43 \quad 29,000 \quad 135,000$

$05 / 19 / 43 \quad 22,000 \quad 190,000$

$1105 / 94 \quad 19,600 \quad 95,800$

$\begin{array}{lll}4 / 19 / 76 & 6,170 \quad 43,000\end{array}$

$06 / 16 / 55 \quad 1,070 \quad 9,910$

$\begin{array}{lll}1,1,640 & 10,000 \\ 10 / 1 / 1 / 73 & 8,730 & 31,800\end{array}$

$\begin{array}{lll}10 / 10 / 73 & 13,200 & 43.500\end{array}$

$\begin{array}{lll}10 / 20 / 84 & 4,220 \quad 93,000\end{array}$

$05 / 08 / 93 \quad 12,000 \quad 24,800$

$\begin{array}{lll}05 / 08 / 93 & 10,000 & 18,400\end{array}$

$\begin{array}{lll}05 / 08 / 93 & 8,000 & 13,300\end{array}$

$\begin{array}{lll}05 / 27 / 84 & 5,910 & 13,000\end{array}$

$\begin{array}{lll}05 / 18 / 43 & 23,000 & 99,500\end{array}$

$\begin{array}{lll}05 / 04 / 41 & 200,000 & 535,000\end{array}$

$\begin{array}{lll}05 / 00 / 43 & 17,000 & 96,400\end{array}$

$\begin{array}{lll}09 / 21 / 65 & 6.150 \quad 28,500\end{array}$

$\begin{array}{lll}05 / 20 / 77 & 2,800 & 11.500\end{array}$

$\begin{array}{lll}07 / 23 / 63 & 4,800 & \\ 0 & 35,500\end{array}$

$\begin{array}{lll}10 / 00 / 81 & 3,930 & 35,500\end{array}$

$\begin{array}{lll}05 / 16 / 45 & -3,930 & 35,800\end{array}$

$\begin{array}{lll}0.551 / 45 & 6,820 & 66,600 \\ 05 / 27 / 84 & 4,160 & 12,800\end{array}$

$5 / 27 / 84-501350$

$\begin{array}{rrr}05,040 & 13,500 \\ 0,11,84,45 & 11,000 & 39,500\end{array}$

$\begin{array}{ll}54,000 & 39,500\end{array}$

$\begin{array}{ll}11,000 & 39,500\end{array}$

$\begin{array}{lll} & 40,300 & 144,000 \\ 05 / 10 / 50 & 43,200 & 90,800\end{array}$

$\begin{array}{lll}05 / 18 / 49 & 5,800 & 20,600\end{array}$ streamflow-gaging stations with short periods of record in basins within Oklahoma

W, sites west of 98 degrees 15 minutes longitude; Ck, Creek; Ave, Avenue; SW, southwest; Trib, Tributary; NW, northwest; SE, southeas]

\begin{tabular}{ccccc}
$\begin{array}{c}\text { Site } \\
\text { number } \\
\text { (fig. 2) }\end{array}$ & $\begin{array}{c}\text { Contributing } \\
\text { drainage area } \\
\text { (mi') }\end{array}$ & Latitude & Longitude & $\begin{array}{c}\text { Hydrologic } \\
\text { region } \\
\text { (EE) })\end{array}$ \\
\hline & & & & \\
\hline 353 & 28.7 & 0364521 & 0990539 & $\mathrm{~W}$ \\
354 & 125,516 & 0352058 & 0944816 & $\mathrm{E}$ \\
& & & & \\
355 & 243 & 0353450 & 0990235 & $\mathrm{~W}$ \\
& & & & \\
356 & 2.16 & 0353450 & 0990131 & $\mathrm{~W}$ \\
357 & 0.81 & 0353818 & 0985918 & $\mathrm{~W}$ \\
358 & 195 & 0363753 & 0961429 & $\mathrm{E}$ \\
359 & 22.2 & 0362330 & 0974030 & $\mathrm{E}$ \\
360 & 590 & 0362023 & 0964232 & $\mathrm{E}$ \\
& & & & \\
361 & 74.4 & 0345246 & 0943040 & $\mathrm{E}$ \\
362 & 12.1 & 0350226 & 0953421 & $\mathrm{E}$ \\
363 & 1.62 & 0353233 & 0973546 & $\mathrm{E}$ \\
364 & 1.64 & 0353226 & 0973556 & $\mathrm{E}$ \\
365 & 6.91 & 0362325 & 0975440 & $\mathrm{E}$ \\
& & & & \\
366 & 12.4 & 0362225 & 0975400 & $\mathrm{E}$ \\
367 & 69.1 & 0350121 & 0945639 & $\mathrm{E}$ \\
368 & 4.74 & 0352607 & 0973253 & $\mathrm{E}$ \\
369 & 3.24 & 0352514 & 0973259 & $\mathrm{E}$ \\
370 & 2.24 & 0352422 & 0973315 & $\mathrm{E}$ \\
& & & & \\
371 & 2.17 & 0360720 & 0944930 & $\mathrm{E}$ \\
372 & 82.0 & 0364043 & 0943537 & $\mathrm{E}$ \\
373 & 20,962 & 0351803 & 0973554 & $\mathrm{E}$ \\
374 & 75.4 & 0354753 & 0945118 & $\mathrm{E}$ \\
375 & 11.8 & 0353255 & 0981550 & $\mathrm{~W}$ \\
& & & & \\
376 & 1.90 & 0353356 & 0973326 & $\mathrm{E}$ \\
377 & 8.18 & 0361224 & 0955448 & $\mathrm{E}$ \\
378 & 8.50 & 0342706 & 0961356 & $\mathrm{E}$ \\
379 & 31.4 & 0352815 & 0955414 & $\mathrm{E}$ \\
380 & 2.14 & 0360948 & 0955000 & $\mathrm{E}$ \\
381 & 2.26 & 0360932 & 0954956 & $\mathrm{E}$ \\
382 & 10.0 & 0351303 & 0963048 & $\mathrm{E}$ \\
383 & 353 & 0355013 & 0972625 & $\mathrm{E}$ \\
384 & 247 & 0354636 & 0973245 & $\mathrm{E}$ \\
385 & 65.0 & 0342420 & 0975630 & $\mathrm{E}$ \\
386 & 7.45 & 0350542 & 09984543 & $\mathrm{~W}$ \\
& & & &
\end{tabular}




\begin{tabular}{|c|c|c|c|c|c|c|c|c|c|c|c|}
\hline \multirow[b]{2}{*}{$\begin{array}{c}\text { Site } \\
\text { number } \\
\text { (fig. 2) }\end{array}$} & \multirow[b]{2}{*}{$\begin{array}{l}\text { Station } \\
\text { number }\end{array}$} & \multirow[b]{2}{*}{ Station name and location } & \multicolumn{3}{|c|}{ Documented extreme peak discharges } & \multirow{2}{*}{ 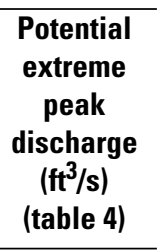 } & \multirow[b]{2}{*}{$\begin{array}{c}\text { Site } \\
\text { number } \\
\text { (fig. 2) }\end{array}$} & \multirow[b]{2}{*}{$\begin{array}{c}\text { Contributing } \\
\text { drainage area } \\
\left(\mathbf{m i}^{2}\right)\end{array}$} & \multirow[b]{2}{*}{ Latitude } & \multirow[b]{2}{*}{ Longitude } & \multirow[b]{2}{*}{$\begin{array}{l}\text { Hydrologic } \\
\text { region } \\
\text { (E/W) }\end{array}$} \\
\hline & & & $\begin{array}{c}\text { Type } \\
\text { basin (N/ } \\
\text { R/U) }\end{array}$ & Date & $\begin{array}{c}\text { Discharge } \\
\left(\mathrm{ft}^{3} / \mathbf{s}\right)\end{array}$ & & & & & & \\
\hline 387 & & Criner Creek near Payne, Okla. & $\mathrm{N}$ & $05 / 17 / 57$ & 12,600 & 85,000 & 387 & 55.0 & 0345303 & 0973140 & $\mathrm{E}$ \\
\hline 388 & & Dead Indian Creek near Roll, Okla. & $\mathrm{N}$ & 04/29/54 & 8,410 & 55,000 & 388 & 33.8 & 0354654 & 0994243 & $\mathrm{w}$ \\
\hline 389 & & Dead Indian Creek near Strong City, Okla. ${ }^{8}$ & $\mathrm{~N}$ & 4/3-4/34 & 17,000 & 69,900 & 389 & 56.0 & 0354056 & 0993750 & $\mathrm{w}$ \\
\hline 390 & & Deep Fork at 34th and Independence, Oklahoma City, Okla. & $\mathrm{U}$ & $11 / 02 / 74$ & 3,940 & 22,000 & 390 & 4.02 & 0353018 & 0973428 & $\mathrm{E}$ \\
\hline 391 & & Deep Fork at NW 39th Street Culvert, Oklahoma City, Okla. & $\mathrm{u}$ & $11 / 02 / 74$ & 4,100 & 28,500 & 391 & 5.83 & 0353041 & 0973323 & $\mathrm{E}$ \\
\hline 392 & 07242200 & Deep Fork at Portland Ave., Oklahoma City, Okla. & $\mathrm{U}$ & 05/20/77 & 4,130 & 17,200 & 392 & 2.98 & 0353006 & 0973458 & $\mathrm{E}$ \\
\hline 393 & & Deep Fork near Stroud, Okla. ${ }^{1}$ & $\mathrm{~N}$ & $05 / 18 / 43$ & 42,000 & 227,000 & 393 & 1,093 & 0354111 & 0963943 & $\mathrm{E}$ \\
\hline 394 & & Deer Creek near Custer City, Okla. ${ }^{1}$ & $\mathrm{~N}$ & $05 / 16 / 51$ & 46,400 & 86,600 & 394 & 90.2 & 0354155 & 0984940 & $\mathrm{w}$ \\
\hline 395 & & Deer Creek near Deer Creek, Okla. ${ }^{2}$ & $\mathrm{~N}$ & 10/11/73 & 36,400 & 83,900 & 395 & 53.1 & 0364808 & 0973010 & $\mathrm{E}$ \\
\hline 396 & & Deer Creek near Hydro, Okla. & $\mathrm{N}$ & 06/00/48 & 70,000 & 138,000 & 396 & 308 & 0353234 & 0983206 & $\mathrm{w}$ \\
\hline 397 & & Deer Creek Tributary near Custer City, Okla. ${ }^{1}$ & $\mathrm{~N}$ & $05 / 16 / 51$ & 7,030 & 19,100 & 397 & 6.74 & 0354112 & 0985036 & $\mathrm{w}$ \\
\hline 398 & & Deer Creek west of Hydro, Okla. $^{1}$ & $\mathrm{~N}$ & 06/22/48 & 31,000 & 134,000 & 398 & 280 & 0353233 & 0983518 & $\mathrm{w}$ \\
\hline 399 & 07334440 & Delaware Creek near Wapanucka, Okla. ${ }^{5}$ & $\mathrm{~N}$ & 10/00/11 & 13,700 & 78,700 & 399 & 45.8 & 0342430 & 0962515 & $\mathrm{E}$ \\
\hline 400 & & Dirty Butter Creek Tributary at Mohawk Road at Tulsa, Okla. ${ }^{4}$ & $\mathrm{U}$ & $05 / 27 / 84$ & 2,920 & 15,900 & 400 & 2.74 & 0361158 & 0955815 & $\mathrm{E}$ \\
\hline 401 & & Dry Creek near Clinton, Okla. ${ }^{1}$ & $\mathrm{~N}$ & $10 / 04 / 55$ & 8,170 & 26,000 & 401 & 10.3 & 0352830 & 0990245 & $\mathrm{w}$ \\
\hline 402 & 07313566 & Dry Creek near Comanche, Okla. & $\mathrm{N}$ & $05 / 18 / 55$ & 5,320 & 50,700 & 402 & 17.0 & 0342144 & 0980108 & $\mathrm{E}$ \\
\hline 403 & & Dry Creek near Davenport, Okla. ${ }^{1}$ & $\mathrm{~N}$ & 05/00/43 & 20,000 & 122,000 & 403 & 144 & 0354326 & 0964439 & $\mathrm{E}$ \\
\hline 404 & & Eagle Chief Creek near Carmen, Okla. ${ }^{1}$ & $\mathrm{~N}$ & $05 / 16 / 57$ & 31,800 & 138,000 & 404 & 306 & 0363444 & 0983037 & $\mathrm{w}$ \\
\hline 405 & & East Branch Hay Creek near Moorewood, Okla. ${ }^{8}$ & $\mathrm{~N}$ & $04 / 03-04 / 34$ & 6,300 & 12,900 & 405 & 4.00 & 0354509 & 0992339 & $\mathrm{w}$ \\
\hline 406 & & East Branch Quartermaster Creek near Moorewood, Okla. ${ }^{8}$ & $\mathrm{~N}$ & 04/03-04/34 & 54,500 & 61,300 & 406 & 42.0 & 0354333 & 0992109 & $\mathrm{w}$ \\
\hline 407 & & East Branch Sandstone Creek near Elk City, Okla. & $\mathrm{N}$ & $04 / 29 / 54$ & 1,340 & 14,600 & 407 & 4.73 & 0353129 & 0993156 & $\mathrm{w}$ \\
\hline 408 & & East Fork Big Creek near Bowlegs, Okla. 1,6,9 & $\mathrm{N}$ & $04 / 14 / 45$ & 3,000 & 5,640 & 408 & 0.89 & 0351440 & 0963354 & $\mathrm{E}$ \\
\hline 409 & 07337920 & Fifteen Creek near Glover, Okla. & $\mathrm{N}$ & $10 / 31 / 72$ & 968 & 7,640 & 409 & 1.23 & 0340633 & 0945542 & $\mathrm{E}$ \\
\hline 410 & 07155100 & Flagg Springs Tributary near Boise City, Okla. & $\mathrm{N}$ & $08 / 21 / 65$ & 2,700 & 15,500 & 410 & 5.15 & 0364620 & 1024816 & $\mathrm{w}$ \\
\hline 411 & & Four Mile Creek near El Reno, Okla. ${ }^{1}$ & $\mathrm{~N}$ & $11 / 19 / 53$ & 6,390 & 35,900 & 411 & 8.51 & 0353220 & 0975249 & $\mathrm{E}$ \\
\hline 412 & & Greenleaf Lake near Braggs, Okla. & $\mathrm{R}$ & $05 / 10 / 50$ & 20,100 & 101,000 & 412 & 87.0 & 0353653 & 0951023 & $\mathrm{E}$ \\
\hline 413 & & Hackberry Creek near Hardesty, Okla. ${ }^{1}$ & $\mathrm{R}$ & $05 / 16 / 55$ & 22,100 & 95,400 & 413 & 116 & 0363259 & 1010948 & $\mathrm{w}$ \\
\hline 414 & & Hackberry Creek near Waukomis, Okla. & $\mathrm{N}$ & $05 / 16 / 57$ & 16,500 & 66,300 & 414 & 31.0 & 0361513 & 0974802 & $\mathrm{E}$ \\
\hline 415 & & Haikey Creek at 91 st Street in Tulsa, Okla. & $\mathrm{N}$ & $05 / 27 / 84$ & 6,040 & 27,300 & 415 & 5.45 & 0360154 & 0955019 & E \\
\hline 416 & 07316070 & Hickory Creek near Marietta, Okla. ${ }^{5}$ & $\mathrm{~N}$ & 10/13-14/81 & 68,100 & 112,000 & 416 & 116 & 0340046 & 0970459 & $\mathrm{E}$ \\
\hline 417 & & Horsepen Creek near Collinsville, Okla. & $\mathrm{N}$ & $05 / 22 / 53$ & 2,540 & 36,000 & 417 & 8.55 & 0362316 & 0955304 & $\mathrm{E}$ \\
\hline 418 & & Hoyle Creek near Ames, Okla. & $\mathrm{N}$ & $05 / 16 / 57$ & 6,800 & 61,600 & 418 & 26.4 & 0361446 & 0981145 & $\mathrm{E}$ \\
\hline 419 & & Hudson Creek near Narcissa, Okla. ${ }^{1}$ & $\mathrm{~N}$ & $05 / 18 / 43$ & 15,800 & 45,200 & 419 & 13.4 & 0364734 & 0945212 & $\mathrm{E}$ \\
\hline 420 & & Joe Creek at East 71st Street South at Tulsa, Okla. ${ }^{4}$ & $\mathrm{U}$ & $05 / 27 / 84$ & 23,000 & 47,200 & 420 & 14.6 & 0360339 & 0955801 & $\mathrm{E}$ \\
\hline 421 & & Johnson Lake near Warner, Okla. & $\mathrm{R}$ & $05 / 10 / 50$ & 459 & 11,200 & 421 & 1.85 & 0352930 & 0952040 & $\mathrm{E}$ \\
\hline 422 & & Knee Creek near Lahoma, Okla. & $\mathrm{N}$ & $05 / 108 / 50$ & 11,200 & 51,200 & 422 & 17.4 & 0362328 & 0980355 & \\
\hline
\end{tabular}




\begin{tabular}{|c|c|c|c|c|c|c|c|c|c|c|c|}
\hline \multirow[b]{2}{*}{$\begin{array}{c}\text { Site } \\
\text { number } \\
\text { (fig. 2) }\end{array}$} & \multirow[b]{2}{*}{$\begin{array}{l}\text { Station } \\
\text { number }\end{array}$} & \multirow[b]{2}{*}{ Station name and location } & \multicolumn{3}{|c|}{ Documented extreme peak discharges } & \multirow{2}{*}{ 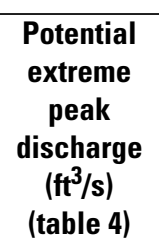 } & \multirow[b]{2}{*}{$\begin{array}{c}\text { Site } \\
\text { number } \\
\text { (fig. 2) }\end{array}$} & \multirow[b]{2}{*}{$\begin{array}{c}\text { Contributing } \\
\text { drainage area } \\
\left(\mathrm{mi}^{2}\right)\end{array}$} & \multirow[b]{2}{*}{ Latitude } & \multirow[b]{2}{*}{ Longitude } & \multirow[b]{2}{*}{$\begin{array}{l}\text { Hydrologic } \\
\text { region } \\
\text { (ENW) }\end{array}$} \\
\hline & & & $\begin{array}{c}\text { Type } \\
\text { basin (N/ } \\
\text { R/U) }\end{array}$ & Date & $\begin{array}{c}\text { Discharge } \\
\left(\mathrm{ft}^{3} / \mathrm{s}\right)\end{array}$ & & & & & & \\
\hline 423 & & Lagoon Ck abv Magnolia Pump Station near Jennings, Okla. ${ }^{1}$ & $\mathrm{~N}$ & $09 / 04 / 40$ & 43,600 & 80,700 & 423 & 48.3 & 0360853 & 0963533 & $\mathrm{E}$ \\
\hline 424 & 07244800 & Lake Eufaula near Brooken, Okla. 10, 11 & $\mathrm{R}$ & 05/00/90 & 430,000 & 627,000 & 424 & 37,822 & 0351825 & 0952145 & $\mathrm{E}$ \\
\hline 425 & 07336600 & Lake Hugo near Hugo, Okla. ${ }^{10,11}$ & $\mathrm{~N}$ & 05/00/90 & 120,000 & 259,000 & 425 & 1,709 & 0340042 & 0952249 & $\mathrm{E}$ \\
\hline 426 & & Lake McGee Creek near Farris, Okla. ${ }^{10,11}$ & $\mathrm{~N}$ & $05 / 00 / 90$ & 32,000 & 131,000 & 426 & 178 & 0341852 & 0955230 & $\mathrm{E}$ \\
\hline 427 & & Lake of the Arbuckles near Dougherty, Okla. ${ }^{10,12}$ & $\mathrm{R}$ & 10/08-09/70 & 80,000 & 116,000 & 427 & 126 & 0342550 & 0970150 & $\mathrm{E}$ \\
\hline 428 & 07335775 & Lake Sardis near Clayton, Okla. ${ }^{10,11}$ & $\mathrm{~N}$ & $05 / 00 / 90$ & 114,000 & 150,000 & 428 & 275 & 0343745 & 0952103 & $\mathrm{E}$ \\
\hline 429 & 07197500 & Lake Tenkiller near Gore, Okla. ${ }^{10,11}$ & $\mathrm{~N}$ & 05/00/90 & 70,000 & 255,000 & 429 & 1,610 & 0353548 & 0950257 & $\mathrm{E}$ \\
\hline 430 & 07331500 & Lake Texoma near Denison, Tex. ${ }^{10,11}$ & $\mathrm{R}$ & $05 / 00 / 90$ & 300,000 & 608,000 & 430 & 33,784 & 0334905 & 0963420 & $\mathrm{E}$ \\
\hline 431 & & Lake Wewoka near Wewoka, Okla. ${ }^{6}$ & $\mathrm{R}$ & $04 / 14 / 45$ & 9,700 & 49,300 & 431 & 16.0 & 0351037 & 0963137 & $\mathrm{E}$ \\
\hline 432 & 07248000 & Lake Wister near Wister, Okla. ${ }^{10,11}$ & $\mathrm{~N}$ & 05/00/90 & 100,000 & 222,000 & 432 & 993 & 0345612 & 0944310 & $\mathrm{E}$ \\
\hline 433 & & Lariat Creek west of Geary, Okla. & $\mathrm{N}$ & $06 / 22 / 48$ & 2,410 & 3,440 & 433 & 0.84 & 0353641 & 0982540 & $\mathrm{w}$ \\
\hline 434 & & Lightning Creek at Grand Avenue at Oklahoma City, Okla. ${ }^{3}$ & $\mathrm{u}$ & 05/08/93 & 15,000 & 44,000 & 434 & 12.7 & 0352541 & 0973046 & $\mathrm{E}$ \\
\hline 435 & & Lightning Creek at Sage Street at Oklahoma City, Okla. ${ }^{3}$ & $\mathrm{u}$ & $05 / 08 / 93$ & 10,000 & 35,700 & 435 & 8.42 & 0352500 & 0973100 & $\mathrm{E}$ \\
\hline 436 & & Lightning Creek at SE 25th Street at Oklahoma City, Okla. ${ }^{3}$ & $\mathrm{U}$ & 05/08/93 & 15,000 & 46,100 & 436 & 13.9 & 0352621 & 0973043 & $\mathrm{E}$ \\
\hline 437 & & Lightning Creek at SW 29th Street at Oklahoma City, Okla. ${ }^{3}$ & $\mathrm{u}$ & 05/08/93 & 15,000 & 45,500 & 437 & 13.6 & 0352606 & 0973050 & $\mathrm{E}$ \\
\hline 438 & & Lightning Creek at SW 44th Street at Oklahoma City, Okla. ${ }^{3}$ & $\mathrm{U}$ & 05/08/93 & 10,000 & 41,800 & 438 & 11.4 & 0352514 & 0973050 & $\mathrm{E}$ \\
\hline 439 & & Lightning Creek at SW 51 st Street at Oklahoma City, Okla. ${ }^{3}$ & $\mathrm{u}$ & 05/08/93 & 10,000 & 35,000 & 439 & 8.12 & 0352451 & 0973105 & $\mathrm{E}$ \\
\hline 440 & & Lightning Creek at SW 19th Street in Oklahoma City, Okla. & $\mathrm{u}$ & 05/29/70 & 4,840 & 46,700 & 440 & 14.3 & 0352644 & 0973033 & E \\
\hline 441 & & Lightning Creek at SW 74th Street in Oklahoma City, Okla. & $\mathrm{U}$ & 05/29/70 & 2,720 & 21,700 & 441 & 3.96 & 0352328 & 0973136 & E \\
\hline 442 & & Little Deep Fork near Depew, Okla. & $\mathrm{N}$ & 05/09/43 & 17,000 & 93,400 & 442 & 69.9 & 0354822 & 0962930 & $\mathrm{E}$ \\
\hline 443 & & Little Deer Creek near Thomas, Okla. ${ }^{1}$ & $\mathrm{~N}$ & $05 / 16 / 51$ & 6,230 & 15,100 & 443 & 4.96 & 0354516 & 0984839 & $\mathrm{w}$ \\
\hline 444 & & Long Creek near Freedom, Okla. ${ }^{1}$ & $\mathrm{~N}$ & $05 / 16 / 57$ & 17,300 & 61,300 & 444 & 42.0 & 0364244 & 0990708 & $\mathrm{w}$ \\
\hline 445 & 07333910 & McGee Creek near Farris, Okla. & $\mathrm{N}$ & 03/27/77 & 36,000 & 130,000 & 445 & 176 & 0341854 & 0955230 & E \\
\hline 446 & & Meridian Creek near Sweetwater, Okla. & $\mathrm{N}$ & $04 / 29 / 54$ & 1,210 & 32,100 & 446 & 14.0 & 0352944 & 0995930 & $\mathrm{w}$ \\
\hline 447 & & Middle Boggy Creek at Enid, Okla. & $\mathrm{u}$ & 07/29/50 & 5,830 & 43,400 & 447 & 12.3 & 0362327 & 0975345 & $\mathrm{E}$ \\
\hline 448 & & Mill Creek in McClure Park at Tulsa, Okla. ${ }^{4}$ & $\mathrm{u}$ & $05 / 27 / 84$ & 12,300 & 14,700 & 448 & 2.50 & 0360900 & 0955322 & $\mathrm{E}$ \\
\hline 449 & 07331250 & Mill Creek near Ravia, Okla. ${ }^{13}$ & $\mathrm{~N}$ & 10/08/70 & 15,300 & 102,000 & 449 & 89.2 & 0341535 & 0964837 & $\mathrm{E}$ \\
\hline 450 & 07332950 & Muddy Boggy at Atoka, Okla. ${ }^{5}$ & $\mathrm{~N}$ & $10 / 16 / 81$ & 29,800 & 173,000 & 450 & 445 & 0342323 & 0960712 & $\mathrm{E}$ \\
\hline 451 & 07299720 & Mule Creek near Eldorado, Okla. & $\mathrm{N}$ & 09/20/65 & 1,740 & 12,500 & 451 & 3.84 & 0342700 & 0993210 & $\mathrm{w}$ \\
\hline 452 & & Nine Mile Creek near Hammon, Okla. ${ }^{8}$ & $\mathrm{~N}$ & $04 / 03-04 / 34$ & 36,000 & 61,300 & 452 & 42.0 & 0353831 & 0992754 & $\mathrm{w}$ \\
\hline 453 & & North Boggy Creek at Enid, Okla. ${ }^{2}$ & $\mathrm{u}$ & 10/10/73 & 10,200 & 32,500 & 453 & 7.17 & 0362400 & 0975224 & $\mathrm{E}$ \\
\hline 454 & & North Fork Walnut Creek near Blanchard, Okla. & $\mathrm{N}$ & $05 / 28 / 49$ & 36,400 & 81,400 & 454 & 49.2 & 0350951 & 0973651 & E \\
\hline 455 & & Okmulgee Dam near Okmulgee, Okla. ${ }^{6}$ & $\mathrm{R}$ & $04 / 14 / 45$ & 11,000 & 74,200 & 455 & 40.1 & 0353717 & 0960342 & $\mathrm{E}$ \\
\hline 456 & & Old Channel Boggy Creek at Lahoma Road at Enid, Okla. ${ }^{2}$ & $\mathrm{U}$ & 10/10/73 & 1,660 & 2,380 & 456 & 0.38 & 0362327 & 0975340 & E \\
\hline 457 & & Otter Creek near Roosevelt (at Narrows), Okla. & $\mathrm{N}$ & 06/05//33 & 16,300 & 64,600 & 457 & 47.0 & 0344910 & 0990137 & $\mathrm{w}$ \\
\hline 458 & & Otter Creek Tributary at Roosevelt, Okla. & $\mathrm{N}$ & $06 / 05 / 53$ & 2,670 & 11,500 & 458 & 3.45 & 0345156 & 0990131 & $\mathrm{w}$ \\
\hline
\end{tabular}




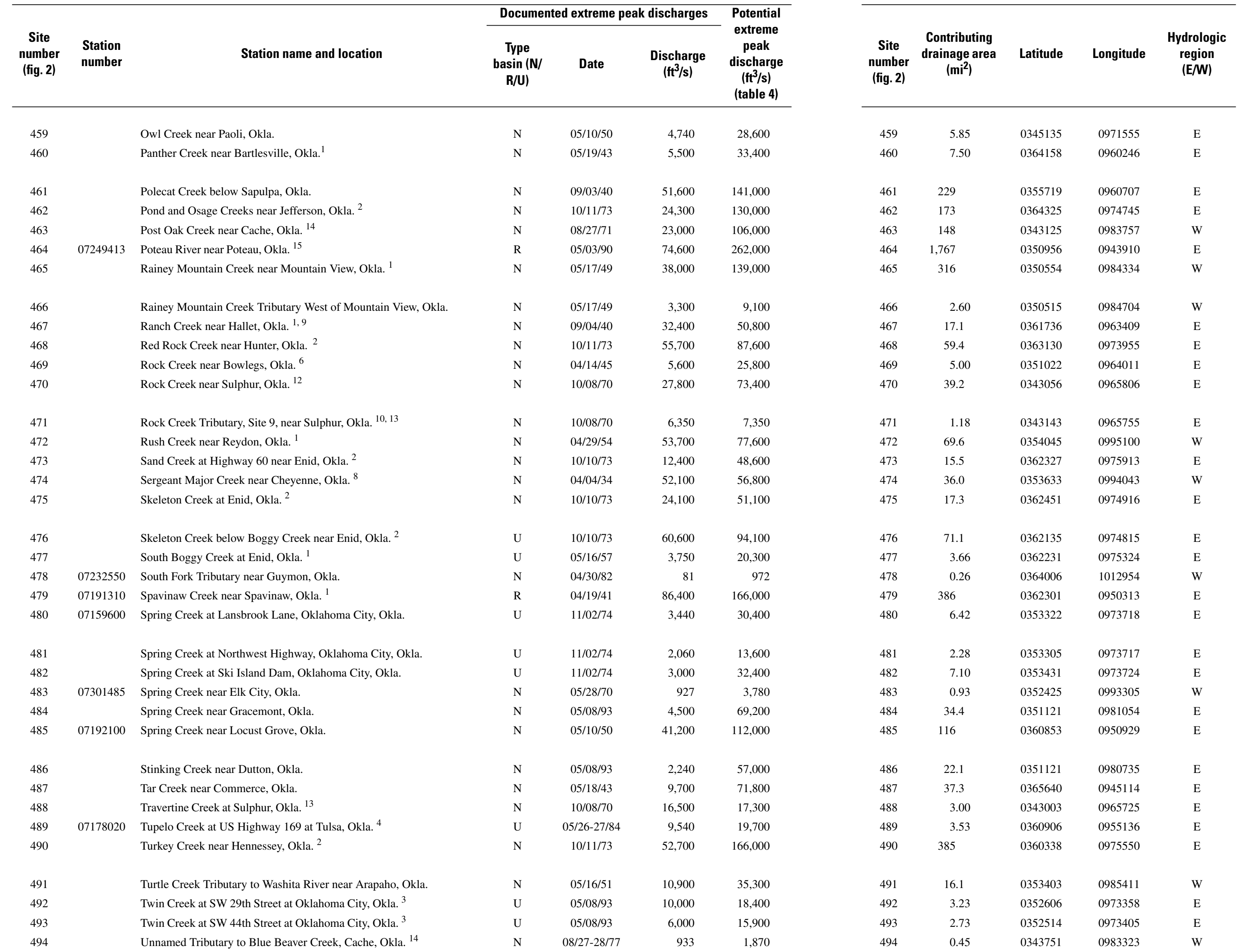


Table 2. Documented and potential extreme peak discharges for selected indirect measurement sites without streamflow-gaging stations and

\begin{tabular}{|c|c|c|c|c|c|c|}
\hline \multirow[b]{2}{*}{$\begin{array}{c}\text { Site } \\
\text { number } \\
\text { (fig. 2) }\end{array}$} & \multirow[b]{2}{*}{$\begin{array}{l}\text { Station } \\
\text { number }\end{array}$} & \multirow[b]{2}{*}{ Station name and location } & \multicolumn{3}{|c|}{ Documented extreme peak discharges } & \multirow{2}{*}{$\begin{array}{c}\text { Potential } \\
\text { extreme } \\
\text { peak } \\
\text { discharge } \\
\text { (ft/3/s) } \\
\text { (table 4) }\end{array}$} \\
\hline & & & $\begin{array}{c}\text { Type } \\
\text { basin (N/ } \\
\text { R/U) }\end{array}$ & Date & $\begin{array}{c}\text { Discharge } \\
\left(\mathrm{ft}^{3} / \mathrm{s}\right)\end{array}$ & \\
\hline 495 & & Unnamed Trib to Quartermaster Ck near Moorewood, Okla. ${ }^{8}$ & $\mathrm{~N}$ & $04 / 03-04 / 34$ & 18,100 & 41,000 \\
\hline 496 & 07171500 & Verdigris River Near Sageeyah, Okla. & $\mathrm{N}$ & $05 / 21 / 43$ & 138,000 & 345,000 \\
\hline 497 & 07315900 & Walnut Bayou near Burneyville, Okla. ${ }^{5}$ & $\mathrm{~N}$ & $10 / 13-14 / 81$ & 29,600 & 156,000 \\
\hline 498 & & Walnut Creek near Lone Grove, Okla. ${ }^{1}$ & $\mathrm{~N}$ & $05 / 17 / 57$ & 63,000 & 118,000 \\
\hline 499 & & Warren Branch near Peoria, Okla. & $\mathrm{N}$ & $05 / 18 / 43$ & 4,200 & 42,900 \\
\hline 500 & & Washita River near Cordell, Okla. ${ }^{8}$ & $\mathrm{~N}$ & $04 / 04 / 34$ & 65,000 & 213,000 \\
\hline 501 & & West Barnitz Creek near Arapaho, Okla. ${ }^{8}$ & $\mathrm{~N}$ & $04 / 03-04 / 34$ & 16,600 & 111,000 \\
\hline 502 & & West Branch Blue Beaver Creek near Cache, Okla. ${ }^{14}$ & $\mathrm{~N}$ & 08/27-28/77 & 6,370 & 14,400 \\
\hline 503 & & West Branch Hay Creek near Moorewood, Okla. ${ }^{8}$ & $\mathrm{~N}$ & $04 / 03-04 / 34$ & 9,400 & 48,700 \\
\hline 504 & & West Branch Quartermaster Creek near Moorewood, Okla. ${ }^{8}$ & $\mathrm{~N}$ & $04 / 03-04 / 34$ & 34,300 & 88,600 \\
\hline 505 & & West Branch Quartermaster Creek near Moorewood, Okla. ${ }^{8}$ & $\mathrm{~N}$ & $04 / 03-04 / 34$ & 67,400 & 92,400 \\
\hline 506 & & West Cache Creek near Cache, Okla. ${ }^{14}$ & $\mathrm{~N}$ & $08 / 28 / 77$ & 13,600 & 73,900 \\
\hline 507 & & West Cache Creek near Faxon, Okla. ${ }^{14}$ & $\mathrm{~N}$ & 08/28/77 & 45,700 & 135,000 \\
\hline 508 & & Wewoka Creek at Lima, Okla. ${ }^{1,6}$ & $\mathrm{~N}$ & $04 / 14 / 45$ & 88,000 & 96,200 \\
\hline 509 & & Wildhorse Creek near Hillsdale, Okla. ${ }^{2}$ & $\mathrm{~N}$ & 10/10/73 & 6,280 & 26,100 \\
\hline 510 & & Wildhorse Creek near Perkins, Okla. & $\mathrm{N}$ & $05 / 20 / 57$ & 23,000 & 72,400 \\
\hline 511 & & Wildhorse Creek near Pond Creek, Okla. ${ }^{2}$ & $\mathrm{~N}$ & 10/11/73 & 102,000 & 112,000 \\
\hline 512 & & Wildhorse Creek near Velma, Okla. & $\mathrm{N}$ & $05 / 19 / 55$ & 19,300 & 111,000 \\
\hline 513 & & Willow Creek at Duncan, Okla. ${ }^{1}$ & $\mathrm{~N}$ & $05 / 10 / 50$ & 5,890 & 21,300 \\
\hline 514 & 07325860 & Willow Creek near Albert, Okla. & $\mathrm{N}$ & 05/20/77 & 12,000 & 50,800 \\
\hline
\end{tabular}

1 Patterson (1964)

Bingham, Bergman, and Thomas, Jr. (1974)

${ }^{4}$ Bergman and Tortorelli (1988)

5 Buckner and Kurklin (1984)

${ }_{7}^{6}$ Bradshaw (1945)

${ }^{7}$ Bradshaw (1945)

${ }_{9}^{8}$ Burnham (1939)

9 Crippen and Bue (1977)

${ }^{11}$ Unflow Calculation

U.S. Army Corps of Engineers, Tulsa District (1990)

${ }^{13}$ U.S. Department of Agriculture, Soil Conservation Service (1970)

${ }^{14}$ Corley and Huntzinger (1979)

\begin{tabular}{ccccc}
$\begin{array}{c}\text { Site } \\
\text { number } \\
\text { (fig. 2) }\end{array}$ & $\begin{array}{c}\text { Contributing } \\
\text { drainage area } \\
\text { (mi } \mathbf{2}^{2} \text { ) }\end{array}$ & Latitude & Longitude & $\begin{array}{c}\text { Hydrologic } \\
\text { region } \\
\text { (E/W) }\end{array}$ \\
\hline & & & & \\
495 & 20.0 & 0354148 & 0991833 & $\mathrm{~W}$ \\
& & & & \\
496 & 4,402 & 0362330 & 0954015 & $\mathrm{E}$ \\
497 & 314 & 0335630 & 0971820 & $\mathrm{E}$ \\
498 & 133 & 0340911 & 0971945 & $\mathrm{E}$ \\
499 & 12.0 & 0365546 & 0943905 & $\mathrm{E}$ \\
500 & 2,160 & 0352058 & 0985126 & $\mathrm{~W}$ \\
& & & & \\
501 & 112 & 0353819 & 09990501 & $\mathrm{~W}$ \\
502 & 4.67 & 0343801 & 0983622 & $\mathrm{~W}$ \\
503 & 27.0 & 0354509 & 0992423 & $\mathrm{~W}$ \\
504 & 61.0 & 0353924 & 0971820 & $\mathrm{E}$ \\
505 & 107 & 0354215 & 0992236 & $\mathrm{~W}$ \\
& & & & \\
506 & 63.1 & 0343609 & 0983757 & $\mathrm{~W}$ \\
507 & 285 & 0342746 & 0983413 & $\mathrm{~W}$ \\
508 & 75.0 & 0351026 & 0963633 & $\mathrm{E}$ \\
509 & 5.08 & 0363305 & 0975440 & $\mathrm{E}$ \\
510 & 38.1 & 0355907 & 0970707 & $\mathrm{E}$ \\
& & & & \\
511 & 116 & 0363723 & 0974736 & $\mathrm{E}$ \\
512 & 112 & 0342906 & 0974144 & $\mathrm{E}$ \\
513 & 3.87 & 0342841 & 0975708 & $\mathrm{E}$ \\
514 & 28.9 & 0351400 & 0982757 & $\mathrm{~W}$ \\
\hline
\end{tabular}

Tortorelli (1996b) 